\title{
ASYMPTOTIC AND NUMERICAL MODELLING OF FLOWS IN FRACTURED POROUS MEDIA
}

\author{
Philippe Angot $^{1}$, Franck Boyer ${ }^{1}$ And Florence Hubert ${ }^{1}$
}

\begin{abstract}
This study concerns some asymptotic models used to compute the flow outside and inside fractures in a bidimensional porous medium. The flow is governed by the Darcy law both in the fractures and in the porous matrix with large discontinuities in the permeability tensor. These fractures are supposed to have a small thickness with respect to the macroscopic length scale, so that we can asymptotically reduce them to immersed polygonal fault interfaces and the model finally consists in a coupling between a 2D elliptic problem and a 1D equation on the sharp interfaces modelling the fractures. A cell-centered finite volume scheme on general polygonal meshes fitting the interfaces is derived to solve the set of equations with the additional differential transmission conditions linking both pressure and normal velocity jumps through the interfaces. We prove the convergence of the FV scheme for any set of data and parameters of the models and derive existence and uniqueness of the solution to the asymptotic models proposed. The models are then numerically experimented for highly or partially immersed fractures. Some numerical results are reported showing different kinds of flows in the case of impermeable or partially/highly permeable fractures. The influence of the variation of the aperture of the fractures is also investigated. The numerical solutions of the asymptotic models are validated by comparing them to the solutions of the global Darcy model or to some analytic solutions.
\end{abstract}

Mathematics Subject Classification. 76S05, 74S10, 35J25, 35J20, 65N15.

Received January 10, 2007. Revised July 29, 2008.

Published online February 7, 2009.

\section{INTRODUCTION}

The present work addresses the numerical modelling of monophasic flows in saturated fractured porous media by means of finite volume methods. The flow in the fracture domain $\Omega_{f}$, in general fully immersed in the porous medium $\Omega$, is assumed to be governed by the Darcy law, as is the flow in the porous matrix, with an anisotropic permeability tensor $\mathbf{K}_{f}$. Our objective is to study asymptotic "double-permeability" models of fracture flow interacting with the matrix flow where the fractures are reduced to sharp interfaces $\Sigma$ when the fracture aperture $b_{f}$ goes to zero. More precisely, if $l_{m}$ and $l_{p}$ denote respectively the macroscopic and pore length scales, we have: $l_{p} \ll b_{f} \ll l_{m}$. The models involve some algebraic or differential immersed transmission conditions on the mean fracture surface $\Sigma$ which combine the jumps of both pressure and normal velocity through the fault interface. The fractures may be "impermeable" (no jump of normal velocity with jumps of pressure on $\Sigma$ ), "highly permeable" (jumps of normal velocity with no jump of pressure on $\Sigma$ ), or characteristic

Keywords and phrases. Fractured porous media, Darcy flow, finite volume method, asymptotic models of flow.

1 Université de Provence and CNRS, Laboratoire d'Analyse Topologie et Probabilités, 39 rue F. Joliot Curie, 13453 Marseille

Cedex 13, France. angot@cmi.univ-mrs.fr; fboyer@cmi.univ-mrs.fr; fhubert@cmi.univ-mrs.fr 
of intermediate cases according to some physical phenomena altering their properties with time: mechanical effects (erosion, sedimentation, clogging, thermomechanical stress), chemical effects... [6,18].

The global Darcy simulations of such problems, typically requiring refined meshes inside the fracture domain $\Omega_{f}$, can be very expensive. Thus, asymptotic models where the fractures are reduced to immersed polygonal fault interfaces, are useful to provide a good approximation of the global flow at a lower cost.

There is a very large literature on the problem of modelling flows in fractured porous media both in the monophasic and multiphasic cases. Reviews on such problems can be found in $[1,6,7,18]$, for instance. Some authors neglect the flow in the porous matrix and only concentrate on the study of the flow in fracture networks (see $[1,7]$ and references therein), others propose to treat the specific geometry of the fractures by a specific numerical method (see for instance $[12,13]$ where joint elements are used). In the diphasic case, where a convection-diffusion-dispersion equation has also to be taken into account, we can refer to the recent reference [24] in which the authors solve their model by using a cell-centered finite volume method.

In the single-phase case we are interested in, our approach follows for instance $[17,21,22]$ and consists in writing an asymptotic model directly on the continuous problem and then to propose an adapted numerical method to treat the obtained simplified system of equations. More precisely, a similar model than the one we propose in Section 2 was studied in $[17,22]$ only in the particular case in which the fracture interface $\Sigma$ is not immersed inside the domain $\widetilde{\Omega}$, but separates it into disjoint subdomains. We propose in this paper the extension of this model to the case of fully immersed fractures and we propose and analyse a corresponding numerical scheme. Our analysis will also be valid for a more general range of values for a quadrature parameter $\xi$ which appears in the model (see Sect. 2.2.5 for a detailed discussion on this point).

\section{Outline}

The paper is organized as follows. Section 2 is devoted to the presentation of the asymptotic models for flow in 2D fractured porous media we are interested in. The permeability anisotropy along the curvilinear coordinates associated with a fracture $\Sigma$ is taken into account. The models depend on some quadrature rules used to approximate the mean of the variables calculated transversely to the fracture and are characterized by a real parameter $\xi \geq \frac{1}{2}$.

We state and prove in Section 3 the global solvability of the asymptotic models in the case of a fully immersed fracture inside the porous matrix and for any value of the parameter $\xi$ in the range $[1 / 2,+\infty[$. In Section 4 , a cell-centered finite volume scheme is proposed to approximate the solution of this problem. We prove the convergence of the finite volume approximate solution towards the unique solution of the asymptotic model under study, for any value of the parameter $\xi \geq 1 / 2$.

Numerical investigations of the validity of the asymptotic models are proposed in Section 5. All the numerical results obtained through the asymptotic models are compared to the solutions of the global double-permeability Darcy system. In order to obtain these reference solutions we use a modified Discrete Duality Finite Volume scheme on meshes which are locally refined in the neighborhood of the fractures. Such m-DDFV schemes were proved to be first-order in the discrete $H^{1}$-norm in [11], for any kind of anisotropy and heterogeneity of the permeability tensor.

We first illustrate the various typical flows we can expect depending on the physical properties of the fracture (impermeable, permeable, ...) in the case of a single fracture and we study the influence of the choice of the quadrature parameter $\xi$.

Then, the behavior of the model and some of its limits are illustrated for more complex situations: a fracture network and a non-constant aperture fracture. Finally, we compare our results with analytical solutions obtained in [23] in the particular case of a lens-shaped fracture in an infinite porous matrix.

\section{Global AND ASYmptotic MOdels FOR FlOWs in 2D FRACTURED POROUS MEDiA}

In the whole paper we consider an open bi-dimensional polygonal bounded domain $\tilde{\Omega} \subset \mathbb{R}^{2}$ representing the porous medium under study. We are interested in the saturated flow of a single incompressible fluid in such 
a porous medium described by the mass conservation equation and the Darcy law. Hence, the main unknowns of the problem are the pressure field $p$ and the related filtration velocity $\mathbf{v}^{p}$.

The boundary $\Gamma \stackrel{\text { def }}{=} \partial \tilde{\Omega}$ is divided into two disjoint subsets $\Gamma=\bar{\Gamma}_{D} \cup \bar{\Gamma}_{N}, \Gamma_{D} \cap \Gamma_{N}=\emptyset$, corresponding to Dirichlet boundary conditions (the pressure is prescribed on $\Gamma_{D}$ ) and to Neumann boundary condition (mass flux is imposed to be 0 on $\Gamma_{N}$ ). It is of course possible to consider more general boundary conditions on $\Gamma_{N}$ like non-homogeneous Fourier boundary conditions for instance but we focus our attention here on homogeneous Neumann conditions.

\subsection{The global Darcy model and its geometry}

We first recall the standard global Darcy model in the case where $\tilde{\Omega}$ is a fractured porous medium. Without any loss of generality we will assume in the analysis that:

- there is a unique fracture in the porous medium. Multiple disjoint fractures can be treated in the very same way. The case of multiple crossing fractures would require some natural supplementary conditions (continuity of the pressure and mass flux balance equation) at the crossing points that we do not tackle in this work;

- the fracture is fully immersed in $\tilde{\Omega}$. As we will see in the numerical results (see Sect. 5), the case where some part of the fracture is in contact with the boundary of the domain can also be considered without additional difficulties.

The geometry is represented in the left part of Figure 1. The porous medium $\tilde{\Omega}$ is split into two subdomains: the porous matrix $\Omega_{m}$ and the fracture $\Omega_{f}$. We assume that the fracture domain $\Omega_{f}$ has the following form

$$
\Omega_{f}=\{s+t \boldsymbol{\nu}(s) / s \in \Sigma, t \in]-\frac{b_{f}(s)}{2}, \frac{b_{f}(s)}{2}[\}
$$

where $\Sigma$ is a 1D polygonal broken line without self-intersection. For any $s \in \Sigma, \boldsymbol{\nu}(s)$ denotes a unit normal vector to $\Sigma$ at the point $s$ (its orientation has no importance at that point and will be precised later), and $b_{f}(s)$ denotes the fracture aperture at the point $s$. Throughout the paper we will assume that

$$
b_{f} \in \mathcal{C}^{1}(\Sigma), \inf _{\Sigma} b_{f}>0
$$

The main physical assumptions we consider are:

- The permeability tensor $\mathbf{K}_{m}$ in the porous matrix $\Omega_{m}$ is constant and isotropic. Since it is isotropic we will often consider $\mathbf{K}_{m}$ as a positive real number.

- The permeability tensor $\mathbf{K}_{f}$ is constant and anisotropic in the fracture. More precisely, we assume that, in the curvilinear frame $(\boldsymbol{\tau}, \boldsymbol{\nu})$ linked to $\Sigma$, we have

$$
\mathbf{K}_{f}=\left[\begin{array}{cc}
\mathbf{K}_{f, \boldsymbol{\tau}} & 0 \\
0 & \mathbf{K}_{f, \boldsymbol{\nu}}
\end{array}\right]
$$

that is $\left(\mathbf{K}_{f} \boldsymbol{\tau}, \boldsymbol{\tau}\right)=\mathbf{K}_{f, \boldsymbol{\tau}},\left(\mathbf{K}_{f} \boldsymbol{\nu}, \boldsymbol{\nu}\right)=\mathbf{K}_{f, \boldsymbol{\nu}}$. Without any additional work it is possible to take into account smooth variations of $\mathbf{K}_{f, \tau}$ and $\mathbf{K}_{f, \nu}$ with respect to the tangential variable $s$ in $\Omega_{f}$, but we will assume here for simplicity that $\mathbf{K}_{f}$ is constant.

Note that we assume that $\mathbf{K}_{f}$ is diagonal in the curvilinear frame, that is $\left(\mathbf{K}_{f} \boldsymbol{\tau}, \boldsymbol{\nu}\right)=0$. This corresponds to many physical situations since the fracture orientation is very likely to be also a characteristic direction of the pore structures inside $\Omega_{f}$. Nevertheless, it is possible to write down a model for general permeabilities such that $\left(\mathbf{K}_{f} \boldsymbol{\tau}, \boldsymbol{\nu}\right) \neq 0$ but its analysis is then much more intricate. Notice that, in practice, the coefficients $\mathbf{K}_{f, \boldsymbol{\tau}}$ and $\mathbf{K}_{f, \boldsymbol{\nu}}$ can be estimated by specific studies of flows inside different kinds of fractures (see [1]). 

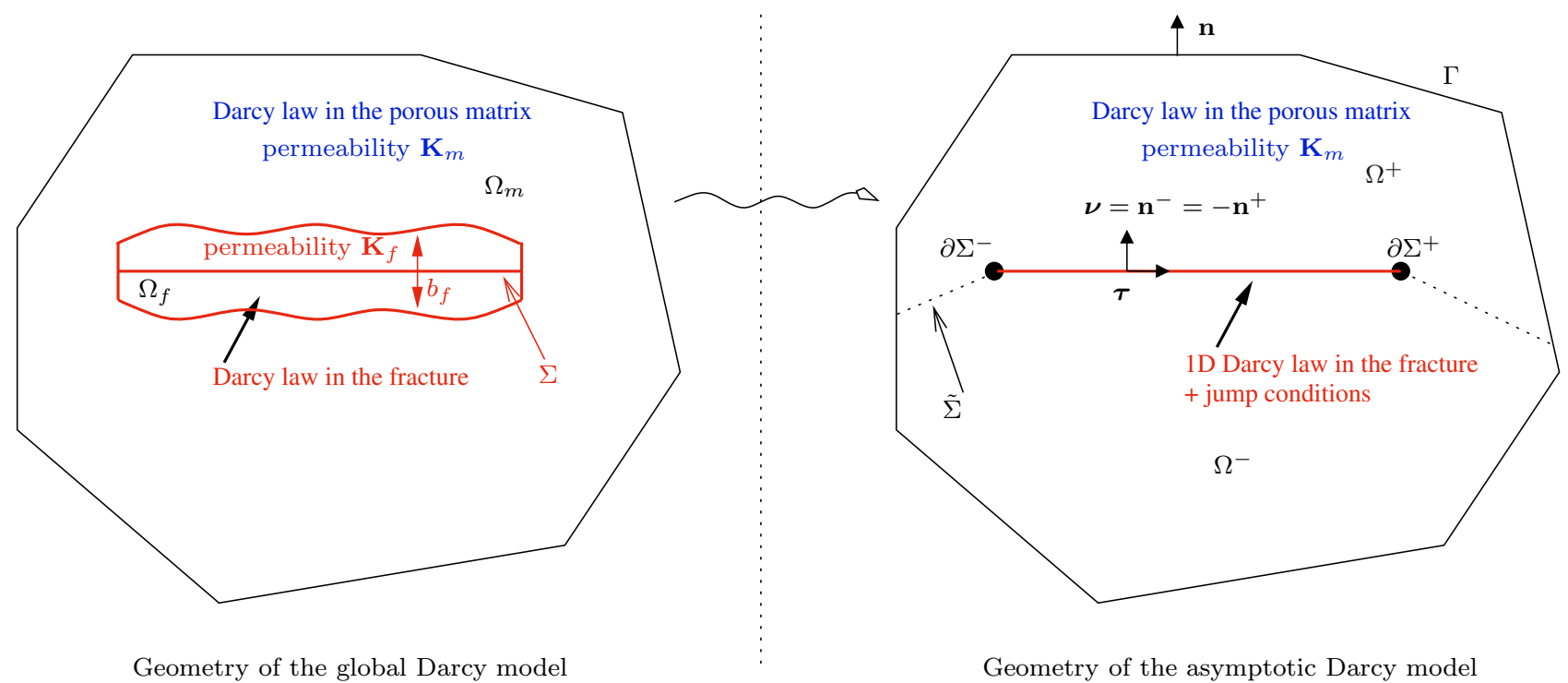

FiguRE 1. Configuration for "double-permeability" models.

With these assumptions at hand, the pressure distribution in the medium is solution to the following global Darcy problem

$$
\begin{aligned}
\nabla \cdot \mathbf{v}^{p} & =Q \text { in } \tilde{\Omega} \\
\mathbf{v}^{p} & =-\frac{1}{\mu} \mathbf{K}(x) \cdot \nabla p \text { in } \tilde{\Omega}, \\
p & =p^{D} \text { on } \Gamma_{D}, \\
\mathbf{v}^{p} \cdot \mathbf{n} & =0 \text { on } \Gamma_{N},
\end{aligned}
$$

where $\mathbf{K}(x)=\mathbf{K}_{m}$ for $x \in \Omega_{m}$ and $\mathbf{K}(x)=\mathbf{K}_{f}$ for $x \in \Omega_{f}, \mu>0$ is the viscosity of the fluid, $Q$ a mass source term and $p^{D}$ the Dirichlet boundary data for the pressure. Notice that, without any loss of generality, we do not take into account the gravity terms in this problem which leads us to simplified notation throughout the paper.

From now on, we assume that the fracture aperture $b_{f}$ is small compared to the length scale of the fracture, that is $b_{f} \ll|\Sigma|$. Furthermore, we emphasize the fact that the permeability coefficients inside $\Omega_{f}$ can be very different from the porous matrix permeability $\mathbf{K}_{m}$. These properties imply that problem $\left(\mathcal{P}_{\mathcal{G}}\right)$ may be difficult to solve numerically. Our objective is to propose in the sequel an asymptotic model in a simplified geometry aiming at providing a good approximation of the flow in the fractured medium in the considered situation.

\subsection{The asymptotic model and its geometry}

We recall here a possible formal derivation of the asymptotic model for flows in such a fractured porous medium we are interested in.

The main idea is to reduce the $2 \mathrm{D}$ fracture domain $\Omega_{f}$ inside $\tilde{\Omega}$ by the $1 \mathrm{D}$ polygonal broken line $\Sigma$. As a consequence, the porous matrix domain $\Omega_{m}$ will be formally replaced by the (larger) open set $\Omega=\tilde{\Omega} \backslash \Sigma$. Notice that $\tilde{\Omega}=\Omega \cup \Sigma$ and that $\partial \Omega=\Gamma \cup \Sigma$. Note that $\Omega$ is a domain with cuts. In particular, does not lie locally on one side of its boundary near the fracture, which will introduce some technical difficulties (see Sect. 3.1.1).

Let us now give the main notation we will use in the sequel. 


\subsubsection{Notation}

- We first embed $\Sigma$ within a larger polygonal broken line $\tilde{\Sigma} \supset \Sigma$ which divides the domain $\tilde{\Omega}$ into two open disjoint subdomains $\Omega^{-}$and $\Omega^{+}$such that $\tilde{\Omega}=\Omega^{-} \cup \tilde{\Sigma} \cup \Omega^{+}$, see the right part of Figure 1 . Notice that the sets $\tilde{\Sigma}, \Omega^{+}$and $\Omega^{-}$will never appear either in the final set of equations or in the numerical scheme we will propose. They are only needed to fix an orientation and to let us give a precise meaning of some trace operators on $\Sigma$ in Section 3.1.1.

- Let $\mathbf{n}$ be the outward unit normal vector on the boundary $\Gamma$ of the computational domain, and $\boldsymbol{\nu}$ the unit normal vector on $\Sigma$ oriented from $\Omega^{-}$to $\Omega^{+}$. The outward unit normal to $\partial \Omega^{+}$on $\Sigma$ is then $\mathbf{n}^{+}=-\boldsymbol{\nu}$ and the outward unit normal to $\partial \Omega^{-}$on $\Sigma$ is $\mathbf{n}^{-}=\boldsymbol{\nu}$. Let $\boldsymbol{\tau}$ be a unit tangential vector on $\Sigma$ so that $(\boldsymbol{\tau}, \boldsymbol{\nu})$ is positively oriented, and $s$ a normalized curvilinear coordinate parameterizing $\Sigma$ in the direction given by $\tau$. In our bidimensional situation, the boundary of $\Sigma$ is composed of two points $\partial \Sigma=\left\{\partial \Sigma^{+}, \partial \Sigma^{-}\right\}$defined in such a way that $s=0$ in $\partial \Sigma^{-}$and $s=|\Sigma|$ in $\partial \Sigma^{+}$.

- For any function $\psi$ in $H^{1}(\Omega)$, let $\gamma^{+} \psi$ and $\gamma^{-} \psi$ be the traces of $\psi$ on each side of $\Sigma$ (see Sect. 3.1.1 for precise definitions), $\bar{\psi}=\frac{1}{2}\left(\gamma^{+} \psi+\gamma^{-} \psi\right)$ be their arithmetic mean, and $\llbracket \psi \rrbracket=\left(\gamma^{+} \psi-\gamma^{-} \psi\right)$ be their jump across $\Sigma$ oriented by $\boldsymbol{\nu}$. Let $\nabla_{\boldsymbol{\tau}}$ and $\nabla_{\boldsymbol{\tau}}$. denote the tangential gradient and divergence operators along $\Sigma$.

\subsubsection{Governing equations for the flow in the porous matrix}

The first part of this model consists in writing the isotropic homogeneous Darcy law inside the new larger porous matrix domain $\Omega$ :

$$
\begin{aligned}
\nabla \cdot \mathbf{v}^{p} & =Q \text { in } \Omega \\
\mathbf{v}^{p} & =-\frac{1}{\mu} \mathbf{K}_{m} \cdot \nabla p \text { in } \Omega \\
p & =p^{D} \text { on } \Gamma_{D} \\
\mathbf{v}^{p} \cdot \mathbf{n} & =0 \text { on } \Gamma_{N} .
\end{aligned}
$$

We want to emphasize the fact that, contrary to the global Darcy model $\left(\mathcal{P}_{\mathcal{G}}\right)$, this system is posed on the domain $\Omega$, and that the permeability tensor $\mathbf{K}_{m}$ is constant and isotropic. In this set of equations, the properties of the fracture $\Sigma$ are not yet taken into account and of course the system is not closed since we need to prescribe the behavior of the solution on $\Sigma$. This will be done in the following section.

\subsubsection{Averaging the Darcy law across the fracture}

We describe in this section the way to formally derive a supplementary set of equations posed on the fracture $\Sigma$ which will complete the problem (2.4)-(2.7) leading to a well-posed problem which is supposed to be a satisfactory approximation of the solution to the initial global Darcy model.

We recall that the flow inside the fracture domain $\Omega_{f}$ (whose geometry is given in (2.1)) is described by the Darcy law for a permeability tensor $\mathbf{K}_{f}$ given in the curvilinear frame by (2.3).

Transversely to $\Sigma$, we define the following mean quantities of the variables:

$$
\begin{gathered}
\mathbf{u}_{f, \boldsymbol{\tau}}(s)=\frac{1}{b_{f}(s)} \int_{-\frac{b_{f}(s)}{2}}^{\frac{b_{f}(s)}{2}} \mathbf{v}^{p}(s, t) \cdot \boldsymbol{\tau}(s) \mathrm{d} t, \mathbf{u}_{f, \boldsymbol{\nu}}(s)=\frac{1}{b_{f}(s)} \int_{-\frac{b_{f}(s)}{2}}^{\frac{b_{f}(s)}{2}} \mathbf{v}^{p}(s, t) \cdot \boldsymbol{\nu}(s) \mathrm{d} t, \\
\Pi_{f} p(s)=\frac{1}{b_{f}(s)} \int_{-\frac{b_{f}(s)}{2}}^{\frac{b_{f}(s)}{2}} p(s, t) \mathrm{d} t, Q_{f}(s)=\frac{1}{b_{f}(s)} \int_{-\frac{b_{f}(s)}{2}}^{\frac{b_{f}(s)}{2}} Q(s, t) \mathrm{d} t,
\end{gathered}
$$

where $p$ and $\mathbf{v}^{p}$ are solutions to the global Darcy model $\left(\mathcal{P}_{\mathcal{G}}\right)$. 
First, we average the mass conservation equation $\left(\mathcal{P}_{\mathcal{G}}\right.$-a $)$ and the Darcy law $\left(\mathcal{P}_{\mathcal{G}}\right.$-b) over the cross-section of the fracture to obtain:

$$
\begin{aligned}
\nabla_{\boldsymbol{\tau}} \cdot\left(b_{f} \mathbf{u}_{f, \boldsymbol{\tau}}\right)+\llbracket \mathbf{v}^{p} \cdot \boldsymbol{\nu} \rrbracket & =b_{f} Q_{f} \\
\mathbf{u}_{f, \boldsymbol{\tau}} & =-\frac{1}{\mu} \mathbf{K}_{f, \boldsymbol{\tau}} \nabla_{\boldsymbol{\tau}} \Pi_{f} p, \\
\mathbf{u}_{f, \boldsymbol{\nu}} & =-\frac{1}{\mu} \mathbf{K}_{f, \boldsymbol{\nu}} \frac{\llbracket p \rrbracket}{b_{f}}
\end{aligned}
$$

Then, by using the trapezoidal quadrature rule to approximate the mean variables with an error of $\mathcal{O}\left(b_{f}^{2} /|\Sigma|^{2}\right)$, that is

$$
\Pi_{f} p \simeq \bar{p}, \quad \mathbf{u}_{f, \nu} \simeq \overline{\mathbf{v}^{p} \cdot \boldsymbol{\nu}}
$$

we get the first asymptotic model of flow along the fault interface $\Sigma$ :

$$
\begin{aligned}
\nabla_{\boldsymbol{\tau}} \cdot\left(b_{f} \mathbf{u}_{f, \boldsymbol{\tau}}\right) & =b_{f} Q_{f}-\llbracket \mathbf{v}^{p} \cdot \boldsymbol{\nu} \rrbracket \text { in } \Sigma \\
\mathbf{u}_{f, \boldsymbol{\tau}} & =-\frac{1}{\mu} \mathbf{K}_{f, \boldsymbol{\tau}} \nabla_{\boldsymbol{\tau}} \Pi_{f} p, \text { in } \Sigma \\
\Pi_{f} p & =\bar{p}, \text { in } \Sigma \\
\frac{\mathbf{v}^{p} \cdot \boldsymbol{\nu}}{} & =-\frac{1}{\mu} \mathbf{K}_{f, \boldsymbol{\nu}} \frac{\llbracket p \rrbracket}{b_{f}}, \text { in } \Sigma,
\end{aligned}
$$

where, in these equations the jumps and the mean-values of $p$ and $\mathbf{v}^{p} \cdot \boldsymbol{\nu}$ are now the one obtained from the solution of the asymptotic model of flow inside the approximate porous matrix $\Omega$, that is system (2.4)-(2.7).

Finally, we need to close the system with a condition on the boundary $\partial \Sigma$ of the fracture. We will consider a Neumann boundary condition

$$
\mathbf{u}_{f, \boldsymbol{\tau}}=0 \quad \text { on } \partial \Sigma .
$$

This boundary condition states that, since the fracture aperture is small, the mass transfer across the extremities of the fracture can be neglected in front of the transversal one. Other kinds of boundary conditions can of course be considered: in the case where the fracture is touching the exterior boundary $\Gamma_{D}$, it can be natural to impose a Dirichlet boundary condition on the pressure on $\partial \Sigma$. We will see that these boundary conditions are not always accurate and should be replaced by more physical conditions (see some numerical results in Sect. 5.4).

\subsubsection{Generalization for other quadrature rules}

Such models have already been proposed in [17,21]. In fact, in those references other quadrature rules are used in place of the trapezoidal rule to approximate the cross-section mean values of the pressure $\Pi_{f} p$ and of $\mathbf{v}^{p} \cdot \boldsymbol{\nu}$ in (2.8). Following the computations in the above references, we may replace (2.11) by

$$
\Pi_{f} p=\bar{p}+\frac{(2 \xi-1) \mu}{4 \mathbf{K}_{f, \boldsymbol{\nu}}} b_{f} \llbracket \mathbf{v}^{p} \cdot \boldsymbol{\nu} \rrbracket, \text { on } \Sigma \text {, }
$$

where $\xi \geq 1 / 2$ is a quadrature parameter.

For example, the trapezoidal rule (2.11) is recovered when $\xi=1 / 2$ which appears to be the most natural and simplest choice, whereas the use of the mid-point rule gives $\xi=3 / 4$. We give a numerical comparison of the models for various values of this parameter in Section 5 . 


\subsubsection{The final asymptotic model under study}

Gathering the previous equations, we finally obtain the following system of equations that we will analyse in the sequel of the paper:

$$
\begin{aligned}
\nabla \cdot \mathbf{v}^{p} & =Q \text { in } \Omega \\
\mathbf{v}^{p} & =-\frac{1}{\mu} \mathbf{K}_{m} \cdot \nabla p \text { in } \Omega \\
p & =p^{D} \text { on } \Gamma_{D} \\
\mathbf{v}^{p} \cdot \mathbf{n} & =0 \text { on } \Gamma_{N}, \\
\nabla_{\boldsymbol{\tau}} \cdot\left(b_{f} \mathbf{u}_{f, \boldsymbol{\tau}}\right. & =b_{f} Q_{f}-\llbracket \mathbf{v}^{p} \cdot \boldsymbol{\nu} \rrbracket \text { in } \Sigma, \\
\mathbf{u}_{f, \boldsymbol{\tau}} & =-\frac{\mathbf{K}_{f, \boldsymbol{\tau}}}{\mu} \nabla_{\boldsymbol{\tau}} \Pi_{f} p \text { in } \Sigma, \\
\mathbf{u}_{f, \boldsymbol{\tau}} & =0, \text { on } \partial \Sigma, \\
\frac{\mathbf{v}^{p} \cdot \boldsymbol{\nu}}{} & =-\frac{\mathbf{K}_{f, \boldsymbol{\nu}}}{\mu} \frac{\llbracket p \rrbracket}{b_{f}} \text { in } \Sigma, \\
\Pi_{f} p & =\bar{p}+\frac{(2 \xi-1) \mu}{4 \mathbf{K}_{f, \boldsymbol{\nu}}} b_{f} \llbracket \mathbf{v}^{p} \cdot \boldsymbol{\nu} \rrbracket \text { in } \Sigma .
\end{aligned}
$$

The quadrature parameter $\xi \in\left[\frac{1}{2},+\infty\left[\right.\right.$ appearing in $\left(\mathcal{P}_{\mathcal{A}}\right.$-i) is now fixed throughout the paper.

In $[21,22]$ a similar model is studied in the case where $\Sigma$ is not immersed inside the domain $\widetilde{\Omega}$. Furthermore, their theoretical and numerical analysis are valid for values of the quadrature parameter $\xi>1 / 2$ (typically $\xi=3 / 4$ or $\xi=1$ ) so that, in this reference, the most natural model (that is when $\xi=1 / 2$ ) is not taken into account. This is due to the fact that the cases $\xi=1 / 2$ and $\xi>1 / 2$ have a very different mathematical structure (we will see in the sequel that the proofs are often different in the two cases). Hence, the mixed formulation used in these references did not allow to perform the analysis for $\xi=1 / 2$. Note that the model for $\xi=3 / 4$ is also numerically studied in [17] in the case of an isotropic fracture permeability tensor $\mathbf{K}_{f}$. For the case of a fully immersed fracture, the asymptotic model is numerically investigated in $[10]$ for $\xi=3 / 4$.

In $[3-5]$, it is also proposed (in the case $\xi=1 / 2)$ to replace the partial differential equation $\left(\mathcal{P}_{\mathcal{A}^{-}}\right.$e) $-\left(\mathcal{P}_{\mathcal{A}^{-}}\right.$) on the fracture by a simpler algebraic model. More precisely, in these references, equations $\left(\mathcal{P}_{\mathcal{A}^{-}}\right.$a $)-\left(\mathcal{P}_{\mathcal{A}}\right.$-d $)$ and $\left(\mathcal{P}_{\mathcal{A}^{-}} \mathrm{h}\right)-\left(\mathcal{P}_{\mathcal{A}^{-}} \mathrm{i}\right)$ are conserved whereas $\left(\mathcal{P}_{\mathcal{A}^{-}} \mathrm{e}\right)-\left(\mathcal{P}_{\mathcal{A}}\right.$-g $)$ are replaced by

$$
\llbracket \mathbf{v}^{p} \cdot \boldsymbol{\nu} \rrbracket=-\frac{b_{f} \mathbf{K}_{f, \boldsymbol{\tau}}}{\mu}\left(\frac{1}{s}(\bar{p}-P)\right)+b_{f} Q_{f} \quad \text { on } \Sigma,
$$

where $P$ is a given reference pressure at $s=0$. This leads to a simpler but less precise model.

\section{WELL-POSEDNESS OF THE ASYMPTOTIC MODELS}

\subsection{Functional setting}

We denote by $\|\cdot\|_{0, \Omega}$ the $L^{2}$-norm on $\Omega$ and by $\|\cdot\|_{1, \Omega}$ the $H^{1}$-norm on $\Omega$. For any $U \subset \partial \Omega$, let $L^{2}(U)$, $H^{\frac{1}{2}}(U)$ and $H^{1}(U)$ be the standard Lebesgue and Sobolev spaces on $U$ endowed with their respective standard norms $\|\cdot\|_{0, U},\|\cdot\|_{1 / 2, U}$ and $\|\cdot\|_{1, U}$.

\subsubsection{Trace results for fractured domains}

We define $H_{\Gamma}^{1}(\Omega)=\left\{p \in H^{1}(\Omega), \quad p=0\right.$ on $\left.\Gamma\right\}$. Let us state the trace results available for the fractured domain $\Omega$. We denote by $\gamma_{0, D}$ and $\gamma_{0, N}$ the trace operators from $H^{1}(\Omega)$ onto $H^{\frac{1}{2}}\left(\Gamma_{D}\right)$ and $H^{\frac{1}{2}}\left(\Gamma_{N}\right)$ respectively. 
Since the fracture $\Sigma$ is supposed to be immersed in $\Omega$, these operators are classically defined in the same way than in the case of a smooth domain.

We concentrate now on the trace problem on the fracture $\Sigma$. First of all, we define the two linear and continuous trace operators $\gamma^{+}$and $\gamma^{-}$from $H^{1}(\Omega)$ on $H^{\frac{1}{2}}(\Sigma)$, by restricting to $\Sigma$ the values of the standard trace operators $\gamma^{+}$and $\gamma^{-}$defined for the two domains $\Omega^{+}$and $\Omega^{-}$, respectively. This definition does not depend on the way $\Omega^{+}$and $\Omega^{-}$are constructed from the interface $\Sigma$.

The space $H_{00}^{\frac{1}{2}}(\Sigma)$ is the set of the functions $g \in H^{\frac{1}{2}}(\Sigma)$ such that $\frac{|g(s)|^{2}}{s(|\Sigma|-s)} \in L^{1}(\Sigma)$ and is endowed with the norm defined by $\|g\|_{1 / 2,00, \Sigma}^{2}=\|g\|_{1 / 2, \Sigma}^{2}+\int_{0}^{|\Sigma|} \frac{|g(s)|^{2}}{s(|\Sigma|-s)} \mathrm{d} s$. We denote by $H^{-\frac{1}{2}}(\Sigma)$ the dual space of $H^{\frac{1}{2}}(\Sigma)$ and by $\tilde{H}^{-\frac{1}{2}}(\Sigma)$ the dual space of $H_{00}^{\frac{1}{2}}(\Sigma)$.

Following [19], Theorems 1.5.2.3, 1.5.1.3, the techniques in [19], Section 1.7, and also [8,9], applied to the open set $\Omega$ (whose boundary is polygonal with some angles of $2 \pi$ ), we have the following result.

Proposition 3.1. The global trace operator $\gamma_{\Sigma}$ on $\Sigma$ defined by

$$
\gamma_{\Sigma}: p \in H^{1}(\Omega) \mapsto \gamma_{\Sigma}(p)=\left(\gamma^{+}(p), \gamma^{-}(p)\right) \in H^{\frac{1}{2}}(\Sigma) \times H^{\frac{1}{2}}(\Sigma)
$$

is continuous from $H^{1}(\Omega)$ onto the space

$$
\begin{aligned}
T_{\Sigma} & =\left\{\left(g^{+}, g^{-}\right) \in\left(H^{\frac{1}{2}}(\Sigma)\right)^{2}, \quad \int_{0}^{|\Sigma|} \frac{\left|g^{+}(s)-g^{-}(s)\right|^{2}}{s(|\Sigma|-s)} \mathrm{d} s<+\infty\right\} \\
& =\left\{\left(g^{+}, g^{-}\right) \in\left(H^{\frac{1}{2}}(\Sigma)\right)^{2}, \quad g^{+}-g^{-} \in H_{00}^{\frac{1}{2}}(\Sigma)\right\},
\end{aligned}
$$

endowed with the norm

$$
\left\|\left(g^{+}, g^{-}\right)\right\|_{T_{\Sigma}}=\left(\left\|g^{+}\right\|_{1 / 2, \Sigma}^{2}+\left\|g^{-}\right\|_{1 / 2, \Sigma}^{2}+\int_{0}^{|\Sigma|} \frac{\left|g^{+}(s)-g^{-}(s)\right|^{2}}{s(|\Sigma|-s)} \mathrm{d} s\right)^{\frac{1}{2}} .
$$

Furthermore, there exists a continuous linear operator $R_{\Sigma}: T_{\Sigma} \rightarrow H_{\Gamma}^{1}(\Omega)$ which is a right inverse of the global trace operator, that is

Finally, $\mathcal{C}_{c}^{\infty}(\Omega)$ is dense in $\operatorname{ker} \gamma_{\Sigma} \cap H_{\Gamma}^{1}(\Omega)$.

$$
\gamma_{\Sigma} \circ R_{\Sigma}=\operatorname{Id}_{T_{\Sigma}}
$$

The proofs of the various statements of this proposition are contained in the references given above, except for the final density statement. For sake of completeness, we provide a proof of this statement in Appendix A.

With this result at hand, one can define the normal traces on $\Sigma$ of any vector field in $\mathbf{v} \in H_{\text {div }}(\Omega) \stackrel{\text { def }}{=}\{\mathbf{u} \in$ $\left.\left(L^{2}(\Omega)\right)^{d}, \nabla \cdot \mathbf{u} \in L^{2}(\Omega)\right\}$ as an element of the dual space $T_{\Sigma}{ }^{\prime}$ as follows.

Proposition 3.2. For any $\mathbf{v} \in H_{\mathrm{div}}(\Omega)$, the $\operatorname{map} \mathbf{v} \cdot \mathbf{n} \in T_{\Sigma}^{\prime}$ defined by

$$
g=\left(g^{+}, g^{-}\right) \in T_{\Sigma} \mapsto(\mathbf{v} \cdot \mathbf{n})(g) \stackrel{\text { def }}{=} \int_{\Omega} \mathbf{v} \cdot \nabla R_{\Sigma}(g) \mathrm{d} x+\int_{\Omega}(\nabla \cdot \mathbf{v}) R_{\Sigma}(g) \mathrm{d} x,
$$

is linear continuous and does not depend on the choice of the right inverse operator $R_{\Sigma}$. Furthermore, there exists a unique element $\llbracket \mathbf{v} \cdot \boldsymbol{\nu} \rrbracket$ in $H^{-\frac{1}{2}}(\Sigma)$ and a unique element $\overline{\mathbf{v} \cdot \boldsymbol{\nu}}$ in $\tilde{H}^{-\frac{1}{2}}(\Sigma)$ such that

$$
\langle\mathbf{v} \cdot \mathbf{n}, g\rangle_{T_{\Sigma^{\prime}, T_{\Sigma}}}=-\left\langle\llbracket \mathbf{v} \cdot \boldsymbol{\nu} \rrbracket, \frac{g^{+}+g^{-}}{2}\right\rangle_{H^{-\frac{1}{2}}(\Sigma), H^{\frac{1}{2}}(\Sigma)}-\left\langle\overline{\mathbf{v} \cdot \boldsymbol{\nu}},\left(g^{+}-g^{-}\right)\right\rangle_{\tilde{H}^{-\frac{1}{2}}(\Sigma), H_{00}^{\frac{1}{2}}(\Sigma)} .
$$


Proof. The continuity of the map $\mathbf{v} \cdot \mathbf{n}$ immediately follows from the continuity of the operator $R_{\Sigma}$ and the definition of the norm in $H_{\text {div }}(\Omega)$.

By definition of $\nabla \cdot \mathbf{v}$, for any $\phi \in \mathcal{C}_{c}^{\infty}(\Omega)$ we have

$$
\int_{\Omega} \mathbf{v} \cdot \nabla \phi \mathrm{d} x+\int_{\Omega}(\nabla \cdot \mathbf{v}) \phi \mathrm{d} x=0
$$

This formula is still true for any $\phi \in \operatorname{ker} \gamma_{\Sigma} \cap H_{\Gamma}^{1}(\Omega)$ by using the density property in Proposition 3.1. Hence, the definition of $\mathbf{v} \cdot \mathbf{n}$ does not depend on the right inverse operator $R_{\Sigma}$ we choose.

We can now define $\llbracket \mathbf{v} \cdot \boldsymbol{\nu} \rrbracket$, and $\overline{\mathbf{v} \cdot \boldsymbol{\nu}}$ as follows

$$
\begin{aligned}
& \langle\llbracket \mathbf{v} \cdot \boldsymbol{\nu} \rrbracket, \psi\rangle_{H^{-\frac{1}{2}}(\Sigma), H^{\frac{1}{2}}(\Sigma)}=\langle\mathbf{v} \cdot \mathbf{n},(-\psi,-\psi)\rangle_{T_{\Sigma^{\prime}, T_{\Sigma}}}, \quad \forall \psi \in H^{\frac{1}{2}}(\Sigma), \\
& \langle\overline{\mathbf{v} \cdot \boldsymbol{\nu}}, \psi\rangle_{\tilde{H}^{-\frac{1}{2}}(\Sigma), H_{00}^{\frac{1}{2}}(\Sigma)}=\left\langle\mathbf{v} \cdot \mathbf{n},\left(-\frac{\psi}{2}, \frac{\psi}{2}\right)\right\rangle_{T_{\Sigma^{\prime}, T_{\Sigma}}}, \quad \forall \psi \in H_{00}^{\frac{1}{2}}(\Sigma) .
\end{aligned}
$$

It is straightforward to see that the required properties hold.

When $\mathbf{v}$ is smooth enough ( mean-value of $\mathbf{v} \cdot \boldsymbol{\nu}$ across $\Sigma$.

Finally, for any $\phi \in H^{1}(\Omega)$ and any $\mathbf{v} \in H_{\text {div }}(\Omega)$, we have the following Stokes-type formula

$$
\int_{\Omega} \mathbf{v} \cdot \nabla \phi \mathrm{d} x+\int_{\Omega}(\nabla \cdot \mathbf{v}) \phi \mathrm{d} x=\left\langle\mathbf{v} \cdot \mathbf{n}, \gamma_{\Gamma} \phi\right\rangle_{H^{-\frac{1}{2}}(\Gamma), H^{\frac{1}{2}}(\Gamma)}-\langle\llbracket \mathbf{v} \cdot \boldsymbol{\nu} \rrbracket, \bar{\phi}\rangle_{H^{-\frac{1}{2}}(\Sigma), H^{\frac{1}{2}}(\Sigma)}-\langle\overline{\mathbf{v} \cdot \boldsymbol{\nu}}, \llbracket \phi \rrbracket\rangle \tilde{H}^{-\frac{1}{2}(\Sigma), H_{00}^{\frac{1}{2}}(\Sigma)} .
$$

We conclude this section by a density result which will be useful in the sequel, in order to show the convergence of the numerical scheme. The idea is that the set of smooth functions in $\Omega$, constant near the extremities of $\Sigma$ but not necessarily continuous across $\Sigma$ is dense in $H^{1}(\Omega)$.

Proposition 3.3. The space $\mathcal{S}$ defined by

$$
\mathcal{S}=\left\{u \in \mathcal{C}^{\infty}(\Omega) \text {, s.t. } u_{\mid \bar{\Omega}^{ \pm}} \in \mathcal{C}^{\infty}\left(\bar{\Omega}^{ \pm}\right) \text {, and } u \text { is constant near the extremities of } \Sigma\right\},
$$

is dense in $H^{1}(\Omega)$.

This result can be proved following similar lines than the proof of the density statement in Proposition 3.1 which is given in Appendix A.

\subsubsection{Functional spaces}

For any pressure field $q \in H^{1}(\Omega)$ defined inside the porous matrix, let us associate the Darcy velocity $\mathbf{v}^{q} \in\left(L^{2}(\Omega)\right)^{2}$ defined by

$$
\mathbf{v}^{q}=-\frac{\mathbf{K}_{m}}{\mu} \nabla q,
$$

and, in the case where $\mathbf{v}^{q} \in H_{\operatorname{div}}(\Omega)$, we define the fracture pressure $\Pi_{f} q$ on $\Sigma$ by

$$
\Pi_{f} q=\bar{q}+\frac{(2 \xi-1) \mu}{4 \mathbf{K}_{f, \nu}} b_{f} \llbracket \mathbf{v}^{q} \cdot \nu \rrbracket \in H^{-\frac{1}{2}}(\Sigma) .
$$

Notice that the product $b_{f} \llbracket \mathbf{v}^{q} \cdot \boldsymbol{\nu} \rrbracket$ is well defined since $b_{f}$ is supposed to be smooth (see (2.2)). We introduce the space

$$
\mathcal{W}=\left\{q \in H^{1}(\Omega) \text { such that }(2 \xi-1) \mathbf{v}^{q} \in H_{\operatorname{div}}(\Omega),(2 \xi-1) \llbracket \mathbf{v}^{q} \cdot \nu \rrbracket \in L^{2}(\Sigma), \Pi_{f} q \in H^{1}(\Sigma)\right\},
$$


endowed with the norm

$$
\|q\|_{\mathcal{W}}=\left(\|q\|_{1, \Omega}^{2}+\left\|\Pi_{f} q\right\|_{1, \Sigma}^{2}+(2 \xi-1)\left\|\llbracket \mathbf{v}^{q} \cdot \nu \rrbracket\right\|_{0, \Sigma}^{2}\right)^{\frac{1}{2}}
$$

Remark 3.4. When $\xi=1 / 2$, this space reduces to $\mathcal{W}=\left\{q \in H^{1}(\Omega), \Pi_{f} q \in H^{1}(\Sigma)\right\}$ with the corresponding norm and, in that case, $\mathcal{W}$ is an Hilbert space, which is not the case when $\xi>1 / 2$.

\subsection{Well-posedness of the problem $\left(\mathcal{P}_{\mathcal{A}}\right)$}

From now on, $p^{D} \in H^{\frac{1}{2}}\left(\Gamma_{D}\right)$ is a given boundary data for the pressure. We call a solution of the asymptotic model, any function $p \in \mathcal{W}$ that satisfies

$$
\begin{aligned}
\nabla \cdot \mathbf{v}^{p} & =Q \text { in } \Omega, \\
p & =p^{D} \text { on } \Gamma_{D}, \\
\mathbf{v}^{p} \cdot \mathbf{n} & =0 \text { on } \Gamma_{N}, \\
-\nabla_{\boldsymbol{\tau}} \cdot\left(b_{f} \frac{\mathbf{K}_{f, \boldsymbol{\tau}}}{\mu} \nabla_{\boldsymbol{\tau}} \Pi_{f} p\right) & =b_{f} Q_{f}-\llbracket \mathbf{v}^{p} \cdot \boldsymbol{\nu} \rrbracket \text { in } \Sigma, \\
-\frac{\mathbf{K}_{f, \boldsymbol{\tau}}}{\mu} \nabla_{\boldsymbol{\tau}} \Pi_{f} p & =0 \text { on } \partial \Sigma, \\
\overline{\mathbf{v}^{p} \cdot \boldsymbol{\nu}} & =-\frac{\mathbf{K}_{f, \boldsymbol{\nu}}}{\mu} \frac{\llbracket p \rrbracket}{b_{f}} \text { on } \Sigma,
\end{aligned}
$$

where $\mathbf{v}^{p}$ and $\Pi_{f} p$ are defined in (3.3)-(3.4) above. Equations (3.8) and (3.9) are required to be satisfied in the weak sense, that is in $H^{-1}(\Sigma)$ and $H^{-1 / 2}(\partial \Sigma)$ respectively.

Our first result is the following.

Theorem 3.5. For any $\xi \geq \frac{1}{2}$, the problem (3.5)-(3.10) admits a unique solution $p \in \mathcal{W}$.

Proof.

- Existence: This will be proved in the following section by passing to the limit in the finite volume scheme.

- Uniqueness: The problem being linear, it is enough to show that if $p \in \mathcal{W}$ is a solution to the homogeneous problem

$$
\begin{aligned}
\mathbf{v}^{p} & =-\frac{\mathbf{K}_{m}}{\mu} \nabla p, \text { in } \Omega, \\
\nabla \cdot \mathbf{v}^{p} & =0 \text { in } \Omega, \\
p & =0, \text { on } \Gamma_{D}, \\
\mathbf{v}^{p} \cdot \mathbf{n} & =0 \text { on } \Gamma_{N}, \\
-\nabla_{\boldsymbol{\tau}} \cdot\left(b_{f} \frac{\mathbf{K}_{f, \boldsymbol{\tau}}}{\mu} \nabla_{\boldsymbol{\tau}} \Pi_{f} p\right) & =-\llbracket \mathbf{v}^{p} \cdot \boldsymbol{\nu} \rrbracket \text { on } \Sigma, \\
-\frac{\mathbf{K}_{f, \boldsymbol{\tau}}}{\mu} \nabla_{\boldsymbol{\tau}} \Pi_{f} p & =0 \text { on } \partial \Sigma, \\
\frac{\mathbf{v}^{p} \cdot \boldsymbol{\nu}}{} & =-\frac{\mathbf{K}_{f, \boldsymbol{\nu}}}{\mu} \frac{\llbracket p \rrbracket}{b_{f}} \text { on } \Sigma,
\end{aligned}
$$

then we have $p=0$. 
To this end, we use $p$ as a test function in (3.12), and using the Stokes formula (3.1), we get

$$
\begin{aligned}
& 0=\int_{\Omega} p \nabla \cdot \mathbf{v}^{p} \mathrm{~d} x \\
& =\int_{\Omega} \frac{\mathbf{K}_{m}}{\mu}|\nabla p|^{2} \mathrm{~d} x-\left\langle\llbracket \mathbf{v}^{p} \cdot \boldsymbol{\nu} \rrbracket, \bar{p}\right\rangle_{H^{-\frac{1}{2}}(\Sigma), H^{\frac{1}{2}}(\Sigma)}-\left\langle\overline{\mathbf{v}^{p} \cdot \boldsymbol{\nu}}, \llbracket p \rrbracket\right\rangle_{\tilde{H}^{-\frac{1}{2}}(\Sigma), H_{00}^{\frac{1}{2}}(\Sigma)} \\
& =\int_{\Omega} \frac{\mathbf{K}_{m}}{\mu}|\nabla p|^{2} \mathrm{~d} x-\left\langle\llbracket \mathbf{v}^{p} \cdot \boldsymbol{\nu} \rrbracket, \bar{p}\right\rangle_{H^{-\frac{1}{2}}(\Sigma), H^{\frac{1}{2}}(\Sigma)}+\int_{\Sigma} \frac{\mathbf{K}_{f, \boldsymbol{\nu}}}{\mu}\left|\frac{\llbracket p \rrbracket}{b_{f}(s)}\right|^{2} b_{f}(s) \mathrm{d} s .
\end{aligned}
$$

Since $p \in \mathcal{W}$, we can use $\Pi_{f} p \in H^{1}(\Sigma)$ as a test function in (3.15) with the boundary condition (3.16). We get

$$
0=\int_{\Sigma} \frac{\mathbf{K}_{f, \boldsymbol{\tau}}}{\mu}\left|\nabla_{\boldsymbol{\tau}} \Pi_{f} p\right|^{2} b_{f} \mathrm{~d} s+\left\langle\llbracket \mathbf{v}^{p} \cdot \boldsymbol{\nu} \rrbracket, \Pi_{f} p\right\rangle_{H^{-\frac{1}{2}}(\Sigma), H^{\frac{1}{2}}(\Sigma)}
$$

Adding the previous two equalities and using the definition (3.4) of $\Pi_{f} p$, it follows

$$
\int_{\Omega} \frac{\mathbf{K}_{m}}{\mu}|\nabla p|^{2} \mathrm{~d} x+\int_{\Sigma} \frac{\mathbf{K}_{f, \boldsymbol{\tau}}}{\mu}\left|\nabla_{\boldsymbol{\tau}} \Pi_{f} p\right|^{2} b_{f} \mathrm{~d} s+\int_{\Sigma} \frac{\mathbf{K}_{f, \boldsymbol{\nu}}}{\mu}\left|\frac{\llbracket p \rrbracket}{b_{f}}\right|^{2} b_{f} \mathrm{~d} s+\frac{(2 \xi-1) \mu}{4 \mathbf{K}_{f, \boldsymbol{\nu}}} \int_{\Sigma} \llbracket \mathbf{v}^{p} \cdot \boldsymbol{\nu} \rrbracket^{2} b_{f} \mathrm{~d} s=0 .
$$

We conclude, since $\xi \geq \frac{1}{2}$, and using (3.13), that the unique solution $p \in \mathcal{W}$ of (3.12)-(3.18) is $p=0$, which proves that problem (3.5)-(3.10) has at most one solution in $\mathcal{W}$.

Remark 3.6. Existence and uniqueness of a solution to problem (3.5)-(3.10) is proved for a non-immersed fracture (that is when $\tilde{\Omega} \backslash \Sigma$ is not connected) and in the case $\xi>\frac{1}{2}$ in [22] by using a mixed formulation. They obtained the result by showing an ellipticity property as well as an inf-sup inequality for this problem. This ellipticity property is no longer satisfied in the case where $\xi=\frac{1}{2}$ and their numerical results, based on the mixed finite element method, show instabilities in the limit $\xi \rightarrow \frac{1}{2}$.

Nevertheless, the existence result for this problem for $\xi=\frac{1}{2}$ can be proved by using the standard variational formulation satisfied by the pressure in the porous matrix. In the case where $p^{D}=0$ (if not one hase to consider a lift of the Dirichlet data to the whole domain), the formulation reads:

$$
\text { Find } p \in \mathcal{W}_{0} \text { such that } a(p, q)=L(q) \text { for all } q \in \mathcal{W}_{0},
$$

where $\mathcal{W}_{0}=\mathcal{W} \cap \gamma_{0, D}{ }^{-1}(\{0\})$, and $a$ is the bilinear form on $\mathcal{W}_{0}$ defined by

$$
a(p, q)=\int_{\Omega} \frac{\mathbf{K}_{m}}{\mu} \nabla p \cdot \nabla q \mathrm{~d} x+\int_{\Sigma} \frac{\mathbf{K}_{f, \boldsymbol{\tau}}}{\mu} \nabla_{\tau} \Pi_{f} p \nabla_{\boldsymbol{\tau}} \Pi_{f} q b_{f} \mathrm{~d} s+\int_{\Sigma} \frac{\mathbf{K}_{f, \boldsymbol{\nu}}}{\mu} \frac{\llbracket p \rrbracket}{b_{f}} \frac{\llbracket q \rrbracket}{b_{f}} b_{f} \mathrm{~d} s
$$

and $L$ is the linear form

$$
L(q)=\int_{\Omega} Q q \mathrm{~d} x+\int_{\Sigma} b_{f} Q_{f} \Pi_{f} q \mathrm{~d} s
$$

Since $\xi=1 / 2, \mathcal{W}_{0}$ is a Hilbert space (see Rem. 3.4). The bilinear form $a$ is then continuous and coercive on $\mathcal{W}_{0}$ and the linear form $L$ is continuous on $\mathcal{W}_{0}$ so that the Lax-Milgram theorem applies. We can check that the solution $p$ to this variational formulation actually solves the problem under study (see the end of the proof of Thm. 4.11).

\section{Finite volume scheme for the asymptotic model $\left(\mathcal{P}_{\mathcal{A}}\right)$}

We recall that we consider in this paper the case where $\mathbf{K}_{m}$ is isotropic. Under this assumption the framework of cell-centered finite volume method on the so-called orthogonal meshes is well adapted. 


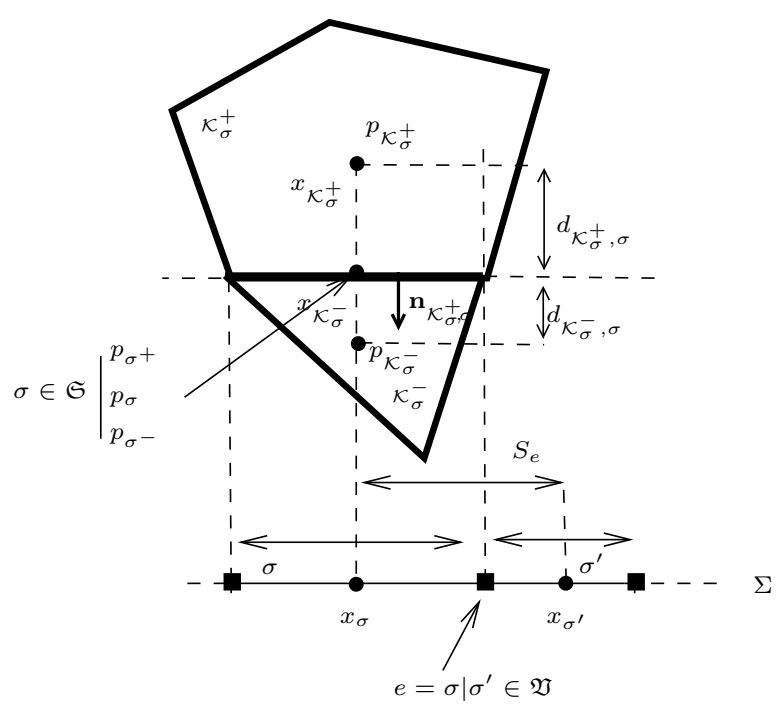

Figure 2. Geometry of the meshes along $\Sigma$.

\subsection{Notation and assumptions for the polygonal mesh}

Let us define the notation we will use to describe and analyze our finite volume scheme. Most of the notation is inspired by that in [16], which is our reference for a general description and analysis of finite volume schemes for standard elliptic equations.

A mesh of the fractured domain $\Omega \cup \Sigma$ is denoted by $\mathcal{T}=(\mathfrak{M}, \mathfrak{S})$ where $\mathfrak{M}$ (resp. $\mathfrak{S}$ ) is a family of disjoint 2-dimensional control volumes $\mathcal{K} \subset \Omega$ (resp. 1-dimensional control volumes $\sigma \subset \Sigma$ ).

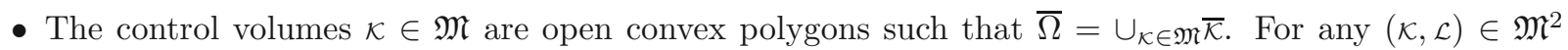
with $\mathcal{K} \neq \mathcal{L}$, either $\overline{\mathcal{K}} \cap \overline{\mathcal{L}}=\emptyset$, a vertex, or $\overline{\mathcal{K}} \cap \overline{\mathcal{L}}=\bar{\sigma}$ for some edge $\sigma \equiv \mathcal{K} \mid \mathcal{L}$.

Let $\mathcal{E}_{\text {int }}$ denote the set of interior edges $\sigma=\mathcal{K} \mid \mathcal{L} \subset \Omega$ and $\mathcal{E}_{\text {ext }}^{D}, \mathcal{E}_{\text {ext }}^{N}$ the sets of edges lying on the boundary $\Gamma$ with $\sigma \subset \Gamma_{D}$ or $\sigma \subset \Gamma_{N}$ respectively. The set $\mathcal{E}$ of all the edges can then be decomposed into $\mathcal{E}=\mathcal{E}_{\text {int }} \cup \mathcal{E}_{\text {ext }}^{D} \cup \mathcal{E}_{\text {ext }}^{N} \cup \mathfrak{S}$.

For each $\mathcal{K} \in \mathfrak{M}$, a discretization point $x_{\mathcal{K}} \in \mathcal{K}$ is chosen such that the segment $\left[x_{\mathcal{K}}, x_{\mathcal{L}}\right]$ is orthogonal at the point $x_{\sigma}$ to each edge $\sigma=\mathcal{K} \mid \mathcal{L}$. This condition is very classical in the framework of cell-centered finite volume schemes for elliptic problems (see [16]). Such meshes are called orthogonal admissible meshes. For a mesh composed by triangles and satisfying the Delaunay condition, it is enough to choose $x_{\mathcal{K}}$ to be the circumcenter of $\mathcal{K}$.

Let $d_{\mathcal{K}, \sigma}>0$ be the distance from $x_{\mathcal{K}}$ to $\sigma$, and $d_{\mathcal{K}, \mathcal{L}}=d_{\mathcal{K}, \sigma}+d_{\mathcal{L}, \sigma}$ the distance between $x_{\mathcal{K}}$ and $x_{\mathcal{L}}$. The set of edges of $\mathcal{K}$ is denoted by $\mathcal{E}_{\mathcal{K}}, \mathbf{n}_{\mathcal{K}}$ is the outward unit normal of $\mathcal{K}$, and for each edge $\sigma \in \mathcal{E}_{\mathcal{K}}$ we will denote more precisely $\mathbf{n}_{\mathcal{K}, \sigma}$ the value of $\mathbf{n}_{\mathcal{K}}$ along $\sigma$. Finally for neighbor control volumes $\mathcal{K}$ and $\mathcal{L}$, $\mathbf{n}_{\mathcal{K}, \mathcal{L}}$ is the unit normal of $\mathcal{K}$ oriented from $\mathcal{K}$ to $\mathcal{L}$.

- We assume that the meshes $\mathfrak{M}$ and $\mathfrak{S}$ are compatible, that is for any control volume $\sigma \in \mathfrak{S}$ there exists $\left(\mathcal{K}_{\sigma}^{+}, \mathcal{K}_{\sigma}^{-}\right) \in \mathfrak{M}^{2}$ such that $\sigma=\mathcal{K}_{\sigma}^{+} \mid \mathcal{K}_{\sigma}^{-}$with $\mathcal{K}_{\sigma}^{+} \subset \Omega^{+}$and $\mathcal{K}_{\sigma}^{-} \subset \Omega^{-}$.

We denote by $\mathfrak{V}$ the set of the vertices $e$ of the mesh $\mathfrak{S}$ and by $\mathfrak{V}_{\text {int }}$ the set of such vertices which are not on the boundary $\partial \Sigma$ (see Fig. 2), so that we have $\mathfrak{V}=\mathfrak{V}_{\text {int }} \cup\left\{\partial \Sigma^{+}, \partial \Sigma^{-}\right\}$. For each $\sigma \in \mathfrak{S}$, let $\mathcal{V}_{\sigma}$ be the set of vertices in $\mathfrak{V}$ belonging to $\partial \sigma$.

To each point $e=\sigma \mid \sigma^{\prime} \in \mathfrak{V}_{\text {int }}$ we associate the segment $S_{e}=\left[x_{\sigma}, x_{\sigma^{\prime}}\right]$ and the unit vector $\boldsymbol{\tau}_{\sigma, \sigma^{\prime}}$ pointing from $x_{\sigma}$ towards $x_{\sigma^{\prime}}$. For $e \in\left\{\partial \Sigma^{-}, \partial \Sigma^{+}\right\}$we note $S_{e}=\left[x_{\sigma}, e\right]$ where $\sigma \in \mathfrak{S}$ is the unique element of $\mathfrak{S}$ such that $e \in \partial \sigma$. We note $\mathfrak{I}=\left(S_{e}\right)_{e \in \mathfrak{V}}$ the set of such segments.

For each $\mathcal{K} \in \mathfrak{M}$ or $\sigma \in \mathfrak{S}, m(\mathcal{K})$ and $m(\sigma)$ denote the 2D-measure of $\mathcal{K}$, resp. the 1D-measure of $\sigma$. The mesh size is defined by: $\operatorname{size}(\mathcal{T})=\sup \{\operatorname{diam}(\mathcal{K}), \mathcal{K} \in \mathfrak{M}\}$. 
Finally, let $r_{\mathcal{K}}>0$ be the largest number $r$ such that the ball of radius $r$ centered at $x_{\mathcal{K}}$ is contained in $\mathcal{K}$. The regularity of the mesh is then measured by the quantity

$$
\operatorname{reg}(\mathcal{T})=\max _{\mathcal{K} \in \mathfrak{M}} \frac{\operatorname{diam}(\mathcal{K})}{r_{\mathcal{K}}},
$$

that we will require to be bounded when the size of the mesh tends to 0 in our convergence results.

\subsection{The discrete spaces}

\subsubsection{The discrete unknowns}

- The matrix pressure field and its traces:

We associate to the mesh $\mathcal{T}$ a set of discrete unknowns $p^{\mathcal{T}}$ composed as follows

$$
p^{\mathcal{T}}=\left(p^{\mathfrak{M}}, \gamma_{0, N} p^{\mathcal{T}}, \gamma^{+} p^{\mathcal{T}}, \gamma^{-} p^{\mathcal{T}}\right) \in E(\mathcal{T}) \stackrel{\text { def }}{=} \mathbb{R}^{\mathfrak{M}} \times \mathbb{R}^{\mathcal{E}_{\text {ext }}^{N}} \times \mathbb{R}^{\mathfrak{S}} \times \mathbb{R}^{\mathfrak{S}}
$$

The unknown vector $p^{\mathfrak{M}}=\left(p_{\mathcal{K}}\right)_{\mathcal{K} \in \mathfrak{M}} \in \mathbb{R}^{\mathfrak{M}}$ contains the cell-centered unknowns on the mesh $\mathfrak{M}$, the vector $\gamma_{0, N} p^{\mathcal{T}}=\left(p_{\sigma}\right)_{\sigma \in \mathcal{E}_{\text {ext }}^{N}}$ represents the boundary values of the pressure on the part of the boundary where Neumann boundary conditions will be imposed. Since we are going to consider possible jumps of the pressure across the fracture $\Sigma$, we need to consider two different discrete traces of the pressure on $\Sigma$ denoted by $\gamma^{+} p^{\mathcal{T}} \stackrel{\text { def }}{=}\left(p_{\sigma^{+}}\right)_{\sigma \in \mathfrak{S}} \in \mathbb{R}^{\mathfrak{S}}$ and $\gamma^{-} p^{\mathcal{T}} \stackrel{\text { def }}{=}\left(p_{\sigma^{-}}\right)_{\sigma \in \mathfrak{S}} \in \mathbb{R}^{\mathfrak{S}}$. The jumps and the mean-value across $\Sigma$ of $p^{\mathcal{T}}$ is defined by $\llbracket p^{\mathcal{T}} \rrbracket=\gamma^{+} p^{\mathcal{T}}-\gamma^{-} p^{\mathcal{T}}$ and $\overline{p^{\mathcal{T}}}=\left(\gamma^{+} p^{\mathcal{T}}+\gamma^{-} p^{\mathcal{T}}\right) / 2$. We can finally define, the boundary value on $\Gamma$ of any given $p^{\mathfrak{M}} \in \mathbb{R}^{\mathcal{T}}$ by $\gamma_{0} p^{\mathfrak{M}}=\left(p_{\mathcal{K}_{\sigma}}\right)_{\sigma \in \mathcal{E}_{\text {ext }}} \in \mathbb{R}^{\mathcal{E}_{\text {ext }}}$, and its restriction on $\Gamma_{D}$

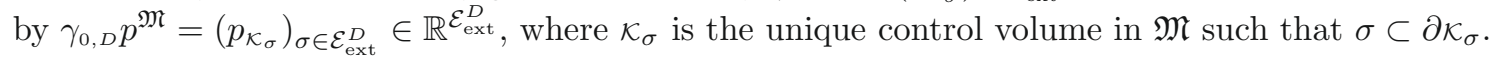

As usual, in order to state our convergence results, discrete functions are identified as piecewise constant functions as follows

$$
\begin{aligned}
p^{\mathfrak{M}} & =\sum_{\mathcal{K} \in \mathfrak{M}} \mathbb{1}_{\mathcal{K}} p_{\mathcal{K}}, \quad \gamma^{+} p^{\mathcal{T}}=\sum_{\sigma \in \mathfrak{S}} \mathbb{1}_{\sigma} p_{\sigma^{+}}, \quad \gamma^{-} p^{\mathcal{T}}=\sum_{\sigma \in \mathfrak{S}} \mathbb{1}_{\sigma} p_{\sigma^{-}}, \\
\gamma_{0, D} p^{\mathfrak{M}} & =\sum_{\sigma \in \mathcal{E}_{\mathrm{ext}}^{D}} \mathbb{1}_{\sigma} p_{\mathcal{K}_{\sigma}}, \quad \gamma_{0, N} p^{\mathcal{T}}=\sum_{\sigma \in \mathcal{E}_{\mathrm{ext}}^{N}} \mathbb{1}_{\sigma} p_{\sigma} .
\end{aligned}
$$

- The fracture pressure:

We associate to the mesh $\mathfrak{S}$ on $\Sigma$, a fracture pressure unknown

$$
p^{\mathfrak{S}}=\left(\left(p_{\sigma}\right)_{\sigma \in \mathfrak{S}}, p_{\partial \Sigma^{-}}, p_{\partial \Sigma^{+}}\right) \in E(\mathfrak{S}) \stackrel{\text { def }}{=} \mathbb{R}^{\mathfrak{S}} \times \mathbb{R} \times \mathbb{R}
$$

where $p_{\sigma}$ is a value at the center $x_{\sigma}$ of the edge $\sigma$ and $p_{\partial \Sigma^{-}}, p_{\partial \Sigma^{+}}$the boundary values at the two extremities $\partial \Sigma^{-}$and $\partial \Sigma^{+}$of $\Sigma$. We associate to $p^{\mathfrak{S}}$ a piecewise constant function on $\Sigma$ still denoted by $p^{\mathfrak{S}}$ and defined $p^{\mathfrak{S}} \stackrel{\text { def }}{=} \sum_{\sigma \in \mathfrak{S}} \mathbb{1}_{\sigma} p_{\sigma}$. Notice that the boundary values $p_{\partial \Sigma^{-}}$and $p_{\partial \Sigma^{+}}$do not enter this definition. Figure 2 sums up the different unknowns introduced near the fracture.

\subsubsection{Discrete gradient}

Let us define the diamond cells $\mathcal{D}_{\sigma}$ for $\sigma \in \mathcal{E}_{\text {int }} \cup \mathcal{E}_{\text {ext }}$ and $\mathcal{D}_{\sigma^{+}}, \mathcal{D}_{\sigma^{-}}$for $\sigma \in \mathfrak{S}$ as shown in Figure 3. For $\sigma=\mathcal{K} \mid \mathcal{L} \in \mathcal{E}_{\text {int }}, \mathcal{D}_{\sigma}$ is the quadrangle whose diagonals are $\sigma$ and $\left[x_{\mathcal{K}}, x_{\mathcal{L}}\right]$. The set of such diamond cells is called $\mathfrak{D}_{\text {int. }}$. For $\sigma=\mathcal{E}_{\text {ext }} \cap \mathcal{E}_{\mathcal{K}}, \mathcal{D}_{\sigma}$ is the triangle defined by the point $x_{\mathcal{K}}$ and the edge $\sigma$. The set of such diamond cells is called $\mathfrak{D}_{\text {ext }}$. Finally, for $\sigma \in \mathfrak{S}, \mathcal{D}_{\sigma^{+}}$and $\mathcal{D}_{\sigma^{-}}$are the two triangles defined by the edge $\sigma$ and by the points $x_{\mathcal{K}_{\sigma}^{+}}$and $x_{\mathcal{K}_{\sigma}^{-}}$respectively. We note $\mathfrak{D}_{\Sigma^{+}}=\left\{\mathcal{D}_{\sigma^{+}}, \sigma \in \mathfrak{S}\right\}, \mathfrak{D}_{\Sigma^{-}}=\left\{\mathcal{D}_{\sigma^{-}}, \sigma \in \mathfrak{S}\right\}$ and $\mathfrak{D}=\mathfrak{D}_{\text {int }} \cup \mathfrak{D}_{\text {ext }} \cup \mathfrak{D}_{\Sigma^{+}} \cup \mathfrak{D}_{\Sigma^{-}}$. 

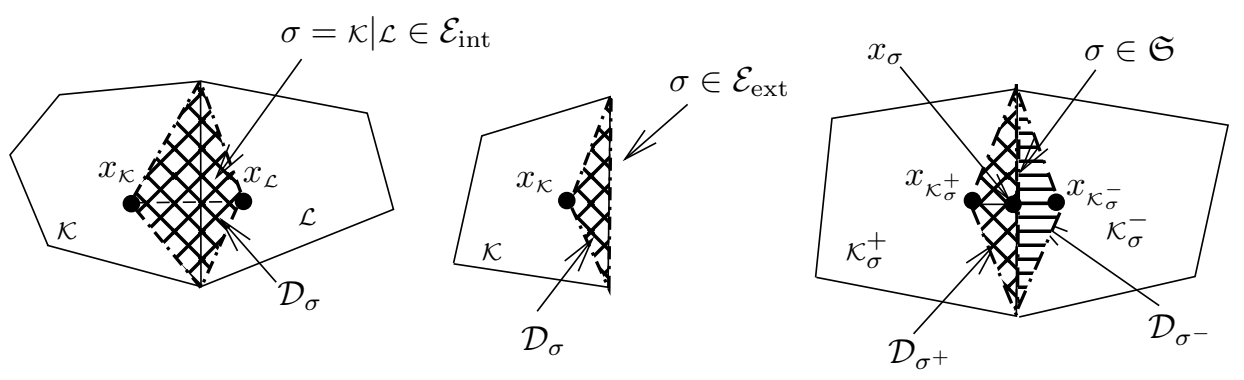

Figure 3. The diamond cells.

Definition 4.1 (discrete gradient on $\mathfrak{M}$ ). For any $p^{\mathcal{T}} \in E(\mathcal{T})$, we define the vector-valued function $\nabla^{\mathfrak{M}, p^{D}} p^{\mathcal{T}}$ by

$$
\begin{aligned}
\nabla^{\mathfrak{M}, p^{D}} p^{\mathcal{T}} \stackrel{\text { def }}{=} 2\left(\sum_{\substack{\mathcal{D}_{\sigma \in \mathfrak{D}_{\text {int }}} \\
\sigma \in \mathcal{K} \mid \mathcal{L}}} \mathbb{1}_{\mathcal{D}_{\sigma}} \frac{p_{\mathcal{L}}-p_{\mathcal{K}}}{d_{\mathcal{K}, \mathcal{L}}} \mathbf{n}_{\mathcal{K}, \mathcal{L}}+\sum_{\substack{\mathcal{D}_{\sigma} \in \mathfrak{D}_{\text {ext }} \\
\sigma \subset \Gamma_{D}}} \mathbb{1}_{\mathcal{D}_{\sigma}} \frac{p_{\sigma}^{D}-p_{\mathcal{K}_{\sigma}}}{d_{\mathcal{K}, \sigma}} \mathbf{n}_{\mathcal{K}_{\sigma}}+\sum_{\substack{\mathcal{D}_{\sigma} \in \mathfrak{D}_{\text {ext }} \\
\sigma \subset \Gamma_{N}}} \mathbb{1}_{\mathcal{D}_{\sigma}} \frac{p_{\sigma}-p_{\mathcal{K}_{\sigma}}}{d_{\mathcal{K}, \sigma}} \mathbf{n}_{\mathcal{K}, \sigma}\right. \\
\left.+\sum_{\mathcal{D}_{\sigma^{+}} \in \mathfrak{D}_{\Sigma^{+}}} \mathbb{1}_{\mathcal{D}_{\sigma^{+}}} \frac{p_{\sigma^{+}}-p_{\mathcal{K}_{\sigma}^{+}}}{d_{\mathcal{K}_{\sigma}^{+}, \sigma}} \mathbf{n}_{\mathcal{K}_{\sigma}^{+}}+\sum_{\mathcal{D}_{\sigma^{-}} \in \mathfrak{D}_{\Sigma^{-}}} \mathbb{1}_{\mathcal{D}_{\sigma^{-}}} \frac{p_{\sigma^{-}}-p_{\mathcal{K}_{\sigma}^{-}}}{d_{\mathcal{K}_{\sigma}^{-}, \sigma}} \mathbf{n}_{\mathcal{K}_{\sigma}^{-}}\right)
\end{aligned}
$$

where $p_{\sigma}^{D}=\frac{1}{m(\sigma)} \int_{\sigma} p^{D}(s) \mathrm{d} s$.

The definition of such a discrete gradient was first proposed in [15] in order to study some links between homogenisation and numerical schemes. Notice that the coefficient 2 in front of the formula (4.1) is in fact the dimension $d=2$ of the problem we are studying. Its presence is due to the fact that only the part of the gradient along the normal $\mathbf{n}_{\sigma}$ is approximated on each diamond cell. As we will see in the proof of Lemma 4.9, this coefficient 2 actually appears to be necessary to reach the weak convergence of the discrete gradient toward the continuous one.

Definition 4.2 (fluxes across $\Sigma$ ). For any $p^{\mathcal{T}} \in E(\mathcal{T})$, we introduce the two mass fluxes across each edge $\sigma$ in $\mathfrak{S}$ by

$$
\mathbf{v}_{\sigma^{+}}^{p^{\mathcal{T}}} \cdot \mathbf{n}^{+} \stackrel{\text { def }}{=}-\frac{\mathbf{K}_{m}}{\mu}\left(\frac{p_{\sigma^{+}}-p_{\mathcal{K}_{\sigma}^{+}}}{d_{\mathcal{K}_{\sigma}^{+}, \sigma}}\right), \quad \mathbf{v}_{\sigma^{-}}^{p^{\mathcal{T}}} \cdot \mathbf{n}^{-\stackrel{\text { def }}{=}}-\frac{\mathbf{K}_{m}}{\mu}\left(\frac{p_{\sigma^{-}}-p_{\mathcal{K}_{\sigma}^{-}}}{d_{\mathcal{K}_{\sigma}^{-}, \sigma}}\right) .
$$

Finally, the jump and the mean-value of these fluxes are defined by

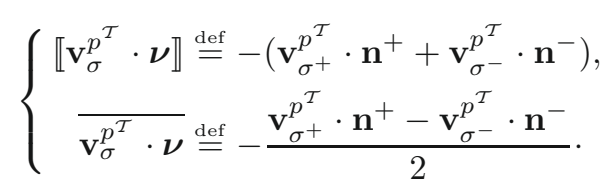

Definition 4.3 (discrete fracture pressure). For any matrix pressure field $p^{\mathcal{T}} \in E(\mathcal{T})$, following (3.4), we define the discrete fracture pressure $\Pi^{\mathfrak{S}} p^{\mathcal{T}} \in E(\mathfrak{S})$ associated to $p^{\mathcal{T}}$ by

$$
\Pi^{\mathfrak{S}} p^{\mathcal{T}}=\frac{1}{2}\left(\gamma^{+} p^{\mathcal{T}}+\gamma^{-} p^{\mathcal{T}}\right)+\frac{(2 \xi-1) \mu}{4 \mathbf{K}_{f, \boldsymbol{\nu}}} b_{f, \sigma} \llbracket \mathbf{v}^{p^{\mathcal{T}}} \cdot \boldsymbol{\nu} \rrbracket,
$$

where $b_{f, \sigma}$ is the mean value of $b_{f}$ on $\sigma$. 
Definition 4.4 (discrete gradient on $\mathfrak{S}$ ). For any fracture pressure field $p^{\mathfrak{S}} \in E(\mathfrak{S})$, we define the real-valued function $\nabla^{\mathfrak{S}} p^{\mathfrak{S}} \in L^{2}(\Sigma)$ by

$$
\nabla^{\mathfrak{S}} p^{\mathfrak{S}}=\sum_{\substack{e \in \mathfrak{V}_{\text {int }} \\ e=\sigma \mid \sigma^{\prime}}} \mathbb{1}_{S_{e}} \frac{p_{\sigma^{\prime}}-p_{\sigma}}{m\left(S_{e}\right)}\left(\boldsymbol{\tau}_{\sigma, \sigma^{\prime}} \cdot \boldsymbol{\tau}\right)+\mathbb{1}_{S_{\partial \Sigma^{+}}} \frac{p_{\partial \Sigma^{+}}-p_{\sigma^{+}}}{m\left(S_{\partial \Sigma^{+}}\right)}-\mathbb{1}_{S_{\partial \Sigma^{-}}} \frac{p_{\partial \Sigma^{-}}-p_{\sigma^{-}}}{m\left(S_{\partial \Sigma^{-}}\right)} .
$$

\subsubsection{The discrete norms}

We define on $E(\mathcal{T})$ the discrete $H^{1}(\Omega)$ norm

$$
\left\|p^{\mathcal{T}}\right\|_{1, \mathcal{T}}=\left(\left\|p^{\mathfrak{M}}\right\|_{0, \Omega}^{2}+\left\|\nabla^{\mathfrak{M}, p^{D}} p^{\mathcal{T}}\right\|_{0, \Omega}^{2}\right)^{\frac{1}{2}}
$$

and on $E(\mathfrak{S})$, the discrete $H^{1}(\Sigma)$ norm

$$
\left\|p^{\mathfrak{S}}\right\|_{1, \mathfrak{S}}=\left(\left\|p^{\mathfrak{S}}\right\|_{0, \Sigma}^{2}+\left\|\nabla^{\mathfrak{S}} p^{\mathfrak{S}}\right\|_{0, \Sigma}^{2}\right)^{\frac{1}{2}}
$$

Finally, we define on $E(\mathcal{T})$ the discrete $W$ norm by

$$
\left\|p^{\mathcal{T}}\right\|_{W^{\mathcal{T}, p^{D}}}=\left(\left\|p^{\mathcal{T}}\right\|_{1, \mathcal{T}}^{2}+\left\|\Pi^{\mathfrak{S}} p^{\mathcal{T}}\right\|_{1, \mathfrak{S}}^{2}+(2 \xi-1)\left\|\llbracket \mathbf{v}^{p^{\mathcal{T}}} \cdot \boldsymbol{\nu} \rrbracket\right\|_{0, \Sigma}^{2}\right)^{\frac{1}{2}} .
$$

Lemma 4.5 (discrete trace inequality). There exists $C>0$ depending on $\operatorname{reg}(\mathcal{T})$ such that for all $p^{\mathcal{T}} \in E(\mathcal{T})$, we have

$$
\left\|\gamma_{0} p^{\mathfrak{M}}\right\|_{0, \Gamma}+\left\|\gamma^{+} p^{\mathcal{T}}\right\|_{0, \Sigma}+\left\|\gamma^{-} p^{\mathcal{T}}\right\|_{0, \Sigma} \leq C\left\|p^{\mathcal{T}}\right\|_{1, \mathcal{T}}
$$

Lemma 4.6 (discrete Poincaré lemma). There exists $C=C(\Omega)$ such that for all $p^{\mathcal{T}} \in E(\mathcal{T})$, we have

$$
\left\|p^{\mathfrak{M}}\right\|_{0, \Omega} \leq C\left(\left\|\nabla^{\mathfrak{M}, p^{D}} p^{\mathcal{T}}\right\|_{0, \Omega}+\left\|p^{D}\right\|_{\frac{1}{2}, \Gamma_{D}}\right)
$$

The proofs of Lemmas 4.5 and 4.6 are direct adaptations of those for the analogous classical results given in $[16]$.

\subsection{The cell-centered numerical scheme for problem $\left(\mathcal{P}_{\mathcal{A}}\right)$}

\subsubsection{Description of the scheme}

- Flow in the porous matrix $\Omega$. Integrating equation (3.5) over each control volumes $\mathcal{K} \in \mathfrak{M}$, the classical cell-centered FV method reads

$$
\sum_{\sigma \in \mathcal{E}_{\mathcal{K}}} F_{\mathcal{K}, \sigma}=m(\mathcal{K}) Q_{\mathcal{K}}, \quad \forall \mathcal{K} \in \mathfrak{M}
$$

where $Q_{\mathcal{K}}$ is the mean value of $Q$ on $\mathcal{K}$, and $F_{\mathcal{K}, \sigma}$ is the numerical flux approximating $\int_{\sigma} \mathbf{v}^{p} \cdot \mathbf{n}_{\mathcal{K}} \mathrm{d} s$. This numerical flux is defined by

$$
F_{\mathcal{K}, \sigma} \stackrel{\text { def }}{=}-m(\sigma) \frac{\mathbf{K}_{m}}{\mu}\left(\frac{p_{\mathcal{K}, \sigma}-p_{\mathcal{K}}}{d_{\mathcal{K}, \sigma}}\right)
$$

where $p_{\mathcal{K}, \sigma}$ is an approximate value of the pressure on the side of $\sigma$ touching the control volume $\mathcal{K}$. Let us see how to determine $p_{\mathcal{K}, \sigma}$. 
- For $\sigma=\mathcal{K} \mid \mathcal{L} \in \mathcal{E}_{\text {int }}$, the pressure is continuous across $\sigma$, that is $p_{\mathcal{K}, \sigma}=p_{\mathcal{L}, \sigma}$, and we have conservation of the fluxes, that is $F_{\mathcal{K}, \sigma}=-F_{\mathcal{L}, \sigma}$. It follows in that case that $p_{\mathcal{K}, \sigma}=p_{\mathcal{L}, \sigma}$ can be eliminated and we get

$$
F_{\mathcal{K}, \mathcal{L}} \stackrel{\text { def }}{=} F_{\mathcal{K}, \sigma}=-F_{\mathcal{L}, \sigma}=-m(\sigma) \frac{\mathbf{K}_{m}}{\mu}\left(\frac{p_{\mathcal{L}}-p_{\mathcal{K}}}{d_{\mathcal{K}, \mathcal{L}}}\right) .
$$

- For $\sigma \in \mathcal{E}_{\text {ext }}^{N}$, then $p_{\mathcal{K}, \sigma}$ is the corresponding value $p_{\sigma}$ of $\gamma_{0, N} p^{\mathcal{T}}$ and the Neumann boundary condition reads $F_{\mathcal{K}_{\sigma}, \sigma}=0$, which determines in fact $p_{\sigma}$. We do not really need its value since the important point is that the numerical flux is zero.

- For $\sigma \in \mathcal{E}_{\text {ext }}^{D}$, then $p_{\mathcal{K}, \sigma}$ is given by the mean value $p_{\sigma}^{D}$ of the Dirichlet data $p^{D}$ on $\sigma$, then

$$
F_{\mathcal{K}_{\sigma}, \sigma}=-m(\sigma) \frac{\mathbf{K}_{m}}{\mu}\left(\frac{p_{\sigma}^{D}-p_{\mathcal{K}_{\sigma}}}{d_{\mathcal{K}_{\sigma}, \sigma}}\right) .
$$

- For $\sigma \in \mathfrak{S}$, then the pressure is not continuous across $\sigma$, and is defined by $\gamma^{ \pm} p^{\mathcal{T}}$ on each side of $\sigma$. More precisely, we have

$$
F_{\mathcal{K}_{\sigma}^{+}, \sigma}=-m(\sigma) \frac{\mathbf{K}_{m}}{\mu}\left(\frac{p_{\sigma^{+}}-p_{\mathcal{K}_{\sigma}^{+}}}{d_{\mathcal{K}_{\sigma}^{+}, \sigma}}\right),
$$

and

$$
F_{\mathcal{K}_{\bar{\sigma}}^{-}, \sigma}=-m(\sigma) \frac{\mathbf{K}_{m}}{\mu}\left(\frac{p_{\sigma^{-}}-p_{\mathcal{K}_{\sigma}^{\bar{\sigma}}}}{d_{\mathcal{K}_{\bar{\sigma}}^{-}, \sigma}}\right) .
$$

In this case, we do not have conservation of the fluxes and the values of $p_{\sigma^{+}}$and $p_{\sigma^{-}}$will be determined through the coupling with the discretization of the 1D elliptic equation (3.8) on $\Sigma$. Note that, using Definition 4.2, we have

$$
F_{\mathcal{K}_{\sigma}^{+}, \sigma}=m(\sigma) \mathbf{v}_{\sigma^{+}}^{p^{\mathcal{T}}} \cdot \mathbf{n}^{+}, \quad F_{\mathcal{K}_{\sigma}^{-}, \sigma}=m(\sigma) \mathbf{v}_{\sigma^{-}}^{p^{\mathcal{T}}} \cdot \mathbf{n}^{-} .
$$

- The discrete fracture pressure. The discrete fracture pressure is now defined by $p^{\mathfrak{S}} \stackrel{\text { def }}{=} \Pi^{\mathfrak{S}} p^{\mathcal{T}}$. In light of Definition 4.3, this yields

$$
p_{\sigma}=\frac{1}{2}\left(p_{\sigma^{+}}+p_{\sigma^{-}}\right)+\frac{(2 \xi-1) \mu}{4 \mathbf{K}_{f, \boldsymbol{\nu}}} b_{f, \sigma} \llbracket \mathbf{v}_{\sigma}^{p^{T}} \cdot \boldsymbol{\nu} \rrbracket, \quad \forall \sigma \in \mathfrak{S} .
$$

- Flow along the fracture $\Sigma$. The 1D finite volume discretization of problem (3.8)-(3.9) reads

$$
\sum_{e \in \mathcal{V}_{\sigma}} G_{\sigma, e}=m(\sigma) b_{f, \sigma} Q_{f, \sigma}-m(\sigma) \llbracket \mathbf{v}_{\sigma}^{p^{\mathcal{T}}} \cdot \boldsymbol{\nu} \rrbracket, \quad \forall \sigma \in \mathfrak{S},
$$

where $Q_{f, \sigma}=\frac{1}{b_{f, \sigma} m(\sigma)} \int_{\sigma} Q_{f}(s) b_{f}(s) \mathrm{d} s$. The numerical flux $G_{\sigma, e}$ approximates $-\frac{\mathbf{K}_{f, \boldsymbol{\tau}}}{\mu} b_{f}\left(\nabla_{\boldsymbol{\tau}} \Pi_{f} p\right)$ at the vertex $e$. This flux is defined by

$$
G_{\sigma, e}=-\frac{\mathbf{K}_{f, \boldsymbol{\tau}}}{\mu} b_{f, e}\left(\frac{p_{\sigma, e}-p_{\sigma}}{d_{\sigma, e}}\right)
$$

where $b_{f, e}$ is the mean value of $b_{f}$ on the segment $\left[x_{\sigma}, x_{\sigma^{\prime}}\right]$ and $p_{\sigma, e}$ is an approximate value of the fracture pressure at the vertex $e$. 
- For $e=\sigma \mid \sigma \in \mathfrak{V}_{\text {int }}$, we use the conservation of the fluxes $G_{\sigma, e}=-G_{\sigma^{\prime}, e}$ and the continuity of the pressure $p_{\sigma, e}=p_{\sigma^{\prime}, e}$, so that the fluxes finally reads

$$
G_{\sigma, \sigma^{\prime}} \stackrel{\text { def }}{=} G_{\sigma, e}=-G_{\sigma^{\prime}, e}=-\frac{\mathbf{K}_{f, \boldsymbol{\tau}}}{\mu} b_{f, e}\left(\frac{p_{\sigma^{\prime}}-p_{\sigma}}{d_{\sigma, \sigma^{\prime}}}\right)
$$

- For $e \in \partial \Sigma=\left\{\partial \Sigma^{+}, \partial \Sigma^{-}\right\}$, the value $p_{\sigma, e}$ is given by $p_{\partial \Sigma^{-}}$or $p_{\partial \Sigma^{+}}$and the Neumann boundary condition (3.9) implies that $G_{\sigma, e}=0$. This uniquely defines the two boundary values $p_{\partial \Sigma^{-}}$and $p_{\partial \Sigma^{+}}$.

- Transmission conditions on $\Sigma$. The discretization of equation (3.10) for all $\sigma=\mathcal{K}_{\sigma}^{+} \mid \mathcal{K}_{\sigma}^{-} \in \mathfrak{S}$ gives:

$$
\overline{\mathbf{v}_{\sigma}^{p^{T}} \cdot \boldsymbol{\nu}}=-\frac{\mathbf{K}_{f, \boldsymbol{\nu}}}{\mu}\left(\frac{p_{\sigma^{+}}-p_{\sigma^{-}}}{b_{f, \sigma}}\right)=-\frac{\mathbf{K}_{f, \boldsymbol{\nu}}}{\mu}\left(\frac{\llbracket p^{\mathcal{T}} \rrbracket_{\sigma}}{b_{f, \sigma}}\right)
$$

If we sum up the above considerations, the finite volume scheme under study consists of the flux balance equations (4.4) and (4.9), together with the flux definitions (4.5), (4.6), (4.7) and (4.10), the definition (4.8) of the fracture pressure and the transmission condition (4.11).

\subsubsection{A priori estimates}

By using the various definitions given above of the discrete gradient operators, we can give a "variational formulation" of the finite volume scheme under study. Notice that, in the finite volume framework, the approximate solutions are piecewise constant and, in particular, are not conformal approximations of the solution in the energy space like for finite element methods.

Lemma 4.7 (Finite Volume variational formulation). Suppose we are given a solution $p^{\mathcal{T}} \in E(\mathcal{T})$ to the scheme (4.4)-(4.11). Then, we have

$\frac{1}{2} \int_{\Omega} \frac{\mathbf{K}_{m}}{\mu} \nabla^{\mathfrak{M}, p^{D}} p^{\mathcal{T}} \cdot \nabla^{\mathfrak{M}, 0} \phi^{\mathcal{T}} \mathrm{d} x+\int_{\Sigma} \frac{\mathbf{K}_{f, \boldsymbol{\nu}}}{\mu} \frac{\llbracket p^{\mathcal{T}} \rrbracket}{b_{f, \sigma}} \frac{\llbracket \phi^{\mathcal{T}} \rrbracket}{b_{f, \sigma}} b_{f}(s) \mathrm{d} s$

$$
-\int_{\Sigma} \overline{\phi^{\mathcal{T}}} \llbracket \mathbf{v}^{p^{\mathcal{T}}} \cdot \boldsymbol{\nu} \rrbracket \mathrm{d} s=\int_{\Omega} Q \phi^{\mathcal{T}} \mathrm{d} x, \quad \forall \phi^{\mathcal{T}} \in E(\mathcal{T})
$$

and

$$
\int_{\Sigma} \frac{\mathbf{K}_{f, \boldsymbol{\tau}}}{\mu} \nabla^{\mathfrak{S}} \Pi^{\mathfrak{S}} p^{\mathcal{T}} \nabla^{\mathfrak{S}} \phi^{\mathfrak{S}} b_{f}(s) \mathrm{d} s=\int_{\Sigma} Q_{f} \phi^{\mathfrak{S}} b_{f} \mathrm{~d} s-\int_{\Sigma} \llbracket \mathbf{v}^{p^{\mathcal{T}}} \cdot \boldsymbol{\nu} \rrbracket \phi^{\mathfrak{S}} \mathrm{d} s, \quad \forall \phi^{\mathfrak{S}} \in E(\mathfrak{S}) .
$$

Proof. Let us multiply equation $(4.4)$ by $\phi_{\mathcal{K}}$ for all $\mathcal{K} \in \mathfrak{M}$ and sum over $\mathfrak{M}$. It follows that

$$
\sum_{\mathcal{K} \in \mathfrak{M}} \sum_{\sigma \in \mathcal{E}_{\mathcal{K}}} F_{\mathcal{K}, \sigma} \phi_{\mathcal{K}}=\sum_{\mathcal{K} \in \mathfrak{M}} m(\mathcal{K}) Q_{\mathcal{K}} \phi_{\mathcal{K}}
$$

We transform now the left-hand side as a sum over the set of edges. For edges $\sigma \in \mathcal{E}_{\text {int }}$ we use the conservation of the fluxes and (4.5). For edges $\sigma \in \mathcal{E}_{\text {ext }}^{D}$ we use definition (4.6) of the flux, and for edges $\sigma \in \mathfrak{S}$, we use 
the two definitions (4.7). We get

$$
\begin{aligned}
\sum_{\mathcal{K} \in \mathfrak{M}} m(\mathcal{K}) Q_{\mathcal{K}} \phi_{\mathcal{K}}= & \sum_{\substack{\sigma \in \mathcal{E}_{\text {int }} \\
\sigma=\mathcal{K} \mid \mathcal{L}}} m(\sigma) \frac{\mathbf{K}_{m}}{\mu}\left(\frac{p_{\mathcal{L}}-p_{\mathcal{K}}}{d_{\mathcal{K}, \mathcal{L}}}\right)\left(\phi_{\mathcal{L}}-\phi_{\mathcal{K}}\right)+\sum_{\sigma \in \mathcal{E}_{\mathrm{ext}}^{D}} m(\sigma) \frac{\mathbf{K}_{m}}{\mu}\left(\frac{p_{\mathcal{K}_{\sigma}}-p_{\sigma}^{D}}{d_{\mathcal{K}_{\sigma}, \sigma}}\right)\left(\phi_{\mathcal{K}_{\sigma}}-0\right) \\
& +\sum_{\sigma \in \mathfrak{S}} m(\sigma) \frac{\mathbf{K}_{m}}{\mu}\left(\frac{p_{\sigma^{+}}-p_{\mathcal{K}_{\sigma}^{+}}}{d_{\mathcal{K}_{\sigma}^{+}, \sigma}}\right)\left(\phi_{\sigma^{+}}-\phi_{\mathcal{K}_{\sigma}^{+}}\right) \\
& +\sum_{\sigma \in \mathfrak{S}} m(\sigma) \frac{\mathbf{K}_{m}}{\mu}\left(\frac{p_{\sigma^{-}}-p_{\mathcal{K}_{\sigma}^{-}}}{d_{\mathcal{K}_{\sigma}^{-}, \sigma}}\right)\left(\phi_{\sigma^{-}}-\phi_{\mathcal{K}_{\sigma}^{-}}\right) \\
& +\sum_{\sigma \in \mathfrak{S}} m(\sigma)\left(\phi_{\sigma^{+}}\left(\mathbf{v}_{\sigma^{+}}^{p^{\mathcal{T}}} \cdot \mathbf{n}^{+}\right)+\phi_{\sigma^{-}}\left(\mathbf{v}_{\sigma^{-}}^{p^{\mathcal{T}}} \cdot \mathbf{n}^{-}\right)\right)
\end{aligned}
$$

Using (4.2), let us rewrite this expression as follows

$$
\begin{aligned}
\sum_{\mathcal{K} \in \mathfrak{M}} m(\mathcal{K}) Q_{\mathcal{K}} \phi_{\mathcal{K}}= & \sum_{\substack{\sigma \in \mathcal{E}_{\text {int }} \\
\sigma=\mathcal{K} \backslash \mathcal{L}}} m(\sigma) d_{\mathcal{K}, \mathcal{L}} \frac{\mathbf{K}_{m}}{\mu}\left(\frac{p_{\mathcal{L}}-p_{\mathcal{K}}}{d_{\mathcal{K}, \mathcal{L}}} \mathbf{n}_{\mathcal{K}, \mathcal{L}}\right) \cdot\left(\frac{\phi_{\mathcal{L}}-\phi_{\mathcal{K}}}{d_{\mathcal{K}, \mathcal{L}}} \mathbf{n}_{\mathcal{K}, \mathcal{L}}\right) \\
& +\sum_{\sigma \in \mathcal{E}_{\text {ext }}^{D}} m(\sigma) d_{\mathcal{K}, \sigma} \frac{\mathbf{K}_{m}}{\mu}\left(\frac{p_{\mathcal{K}}-p_{\sigma}^{D}}{d_{\mathcal{K}, \sigma}} \mathbf{n}_{\mathcal{K}}\right) \cdot\left(\frac{\phi_{\mathcal{K}}-0}{d_{\mathcal{K}, \sigma}} \mathbf{n}_{\mathcal{K}}\right) \\
& +\sum_{\sigma \in \mathfrak{S}} m(\sigma) d_{\mathcal{K}_{\sigma}^{+}, \sigma} \frac{\mathbf{K}_{m}}{\mu}\left(\frac{p_{\sigma^{+}}-p_{\mathcal{K}_{\sigma}^{+}}}{d_{\mathcal{K}_{\sigma}^{+}, \sigma}} \mathbf{n}^{+}\right) \cdot\left(\frac{\phi_{\sigma^{+}}-\phi_{\mathcal{K}_{\sigma}^{+}}}{d_{\mathcal{K}_{\sigma}^{+}, \sigma}} \mathbf{n}^{+}\right) \\
& +\sum_{\sigma \in \mathfrak{S}} m(\sigma) d_{\mathcal{K}_{\sigma}^{-}, \sigma} \frac{\mathbf{K}_{m}}{\mu}\left(\frac{p_{\sigma^{-}}-p_{\mathcal{K}_{\sigma}^{-}}}{d_{\mathcal{K}_{\sigma}^{-}, \sigma}} \mathbf{n}^{-}\right) \cdot\left(\frac{\phi_{\sigma^{-}}-\phi_{\mathcal{K}_{\sigma}^{-}}}{d_{\mathcal{K}_{\sigma}^{-}, \sigma}} \mathbf{n}^{-}\right) \\
& -\sum_{\sigma \in \mathfrak{S}} m(\sigma)\left(\llbracket \phi^{\mathcal{T}} \rrbracket_{\sigma} \overline{\mathbf{v}_{\sigma}^{p^{T}} \cdot \boldsymbol{\nu}}+\overline{\phi_{\sigma}^{\mathcal{T}}} \llbracket \mathbf{v}_{\sigma}^{p^{\mathcal{T}}} \cdot \boldsymbol{\nu} \rrbracket\right) .
\end{aligned}
$$

Since the mesh is conforming, notice that on any interior diamond cell $\mathcal{D}_{\sigma} \in \mathfrak{D}_{\text {int }}$ with $\sigma=\mathcal{K} \mid \mathcal{L}$, we have $m(\sigma) d_{\mathcal{K}, \mathcal{L}}=2 m\left(\mathcal{D}_{\sigma}\right)$, and for any boundary diamond cell $\mathcal{D}_{\sigma} \in \mathfrak{D}_{\text {ext }}$, we have $m(\sigma) d_{\mathcal{K}_{\sigma}, \sigma}=2 m\left(\mathcal{D}_{\sigma}\right)$. Using now the transmission condition (4.11) and Definition 4.1 of the discrete gradient, (4.12) follows.

By similar, and in fact simpler, computations we obtain (4.13).

\section{Corollary 4.8.}

(1) Any solution $p^{\mathcal{T}} \in E(\mathcal{T})$ to the scheme (4.4)-(4.11) satisfies

$$
\begin{aligned}
\int_{\Omega} Q \phi^{\mathcal{T}} \mathrm{d} x+\int_{\Sigma} Q_{f} b_{f} \Pi^{\mathfrak{S}} \phi^{\mathcal{T}} \mathrm{d} s=\frac{1}{2} \int_{\Omega} \frac{\mathbf{K}_{m}}{\mu} \nabla^{\mathfrak{M}, p^{D}} p^{\mathcal{T}} \cdot \nabla^{\mathfrak{M}, 0} \phi^{\mathcal{T}} \mathrm{d} x \\
+\int_{\Sigma} \frac{\mathbf{K}_{f, \boldsymbol{\tau}}}{\mu}\left(\nabla^{\mathfrak{S}} \Pi^{\mathfrak{S}} p^{\mathcal{T}}\right)\left(\nabla^{\mathfrak{S}} \Pi^{\mathfrak{S}} \phi^{\mathcal{T}}\right) b_{f} \mathrm{~d} s+\int_{\Sigma} \frac{\mathbf{K}_{f, \nu}}{\mu} \frac{\llbracket p^{\mathcal{T}} \rrbracket}{b_{f, \sigma}} \frac{\llbracket \phi^{\mathcal{T}} \rrbracket}{b_{f, \sigma}} b_{f} \mathrm{~d} s \\
\quad+\frac{(2 \xi-1) \mu}{4 \mathbf{K}_{f, \nu}} \int_{\Sigma} b_{f} \llbracket \mathbf{v}^{p^{\mathcal{T}}} \cdot \boldsymbol{\nu} \rrbracket \llbracket \mathbf{v}^{\phi^{\mathcal{T}}} \cdot \boldsymbol{\nu} \rrbracket \mathrm{d} s, \quad \forall \phi^{\mathcal{T}} \in E(\mathcal{T}) .
\end{aligned}
$$

(2) Let $\alpha>0$ such that $\operatorname{reg}(\mathcal{T}) \leq \alpha$. For any set of data, there exists a unique such solution $p^{\mathcal{T}} \in E(\mathcal{T})$ and there exists $C>0$ which only depends only on the data $\mu, \mathbf{K}_{m}, \mathbf{K}_{f, \tau}, \mathbf{K}_{f, \nu}, \Omega, b_{f}$ and $\alpha$ such that we have

$$
\left\|p^{\mathcal{T}}\right\|_{W^{\mathcal{T}, p^{D}}} \leq C\left(\|Q\|_{0, \Omega}+\left\|Q_{f}\right\|_{0, \Sigma}+\left\|p^{D}\right\|_{\frac{1}{2}, \Gamma_{D}}+|\mathbf{g}|\right) .
$$


Proof. We obtain (4.14) by taking $\phi^{\mathfrak{S}}=\Pi^{\mathfrak{S}} \phi^{\mathcal{T}}$ in (4.13) and adding it to (4.12).

There exists $R \in H^{1}(\Omega)$ an extension of $p^{D}$ compactly supported in $\bar{\Omega} \backslash \Sigma$, and such that $\|R\|_{1, \Omega} \leq$ $C(\Omega)\left\|p^{D}\right\|_{\frac{1}{2}, \Gamma_{D}}$. We introduce the projection $R^{\mathcal{T}}=\left(R^{\mathfrak{M}}, \gamma_{0, N} R^{\mathcal{T}}, \gamma^{+} R^{\mathcal{T}}, \gamma^{-} R^{\mathcal{T}}\right) \in E(\mathcal{T})$ of $R$ on the mesh $\overline{\mathcal{T}}$, with $R^{\mathfrak{M}}=\left(R_{\mathcal{K}}\right)_{\mathcal{K} \in \mathfrak{M}}$ the mean value projection of $R$ on the mesh $\mathfrak{M}$ that is $R_{\mathcal{K}}$ is the mean-value of $R$ on the ball $B\left(x_{\mathcal{K}}, r_{\mathcal{K}}\right)$ defined in Section 4.1. We naturally choose to take $\gamma^{+} R^{\mathcal{T}}=\gamma^{-} R^{\mathcal{T}}=0$ since $R$ vanishes in the neighborhood of the fracture $\Sigma$, and we take $R_{\sigma}=\frac{1}{m(\sigma)} \int_{\sigma} R \mathrm{~d} s$ for any $\sigma \in \mathcal{E}_{\text {ext }}^{N}$.

We can prove as in $[2,16]$ that

$$
\left\|\nabla^{\mathfrak{M}, p^{D}} R^{\mathcal{T}}\right\|_{0, \Omega} \leq C(\Omega, \operatorname{reg}(\mathcal{T}))\|R\|_{1, \Omega} \leq C\left\|p^{D}\right\|_{\frac{1}{2}, \Gamma_{D}} .
$$

We remark that, if $\operatorname{size}(\mathcal{T})$ is small enough, then $\llbracket \mathbf{v}^{R^{\mathcal{T}}} \cdot \boldsymbol{\nu} \rrbracket=\Pi^{\mathfrak{S}} R^{\mathfrak{M}}=0$. Hence, taking $\phi^{\mathcal{T}}=p^{\mathcal{T}}-R^{\mathcal{T}}$ in (4.14), we obtain

$$
\begin{aligned}
\frac{1}{2} \int_{\Omega} \frac{\mathbf{K}_{m}}{\mu} \nabla^{\mathcal{T}, p^{D}} p^{\mathcal{T}} \cdot\left(\nabla^{\mathfrak{M}, p^{D}} p^{\mathcal{T}}-\nabla^{\mathfrak{M}, p^{D}} R^{\mathcal{T}}\right) \mathrm{d} x+\int_{\Sigma} \frac{\mathbf{K}_{f, \boldsymbol{\tau}}}{\mu} \nabla^{\mathfrak{S}} \Pi^{\mathfrak{S}} p^{\mathcal{T}} \cdot \nabla^{\mathfrak{S}} \Pi^{\mathfrak{S}} p^{\mathcal{T}} b_{f}(s) \mathrm{d} s \\
+\int_{\Sigma} \frac{\mathbf{K}_{f, \boldsymbol{\nu}}}{\mu} \frac{\llbracket p^{\mathcal{T}} \rrbracket}{b_{f, \sigma}} \frac{\llbracket p^{\mathcal{T}} \rrbracket}{b_{f, \sigma}} b_{f}(s) \mathrm{d} s+\frac{(2 \xi-1) \mu}{4 \mathbf{K}_{f, \boldsymbol{\nu}}} \int_{\Sigma} \llbracket \mathbf{v}^{p^{\mathcal{T}}} \cdot \boldsymbol{\nu} \rrbracket^{2} b_{f}(s) \mathrm{d} s= \\
\int_{\Omega} Q(x)\left(p^{\mathfrak{M}}-R^{\mathfrak{M}}\right)(x) \mathrm{d} x+\int_{\Sigma} Q_{f}(s) \Pi^{\mathfrak{S}} p^{\mathcal{T}}(s) b_{f}(s) \mathrm{d} s .
\end{aligned}
$$

We deduce by using Cauchy-Schwarz's and Young's inequalities that

$$
\left\|p^{\mathcal{T}}\right\|_{W^{\mathcal{T}, p D}}^{2} \leq C\left(\|Q\|_{0, \Omega}\left\|p^{\mathfrak{M}}-R^{\mathfrak{M}}\right\|_{0, \Omega}+\left\|R^{\mathcal{T}}\right\|_{1, \mathcal{T}}^{2}+\left\|Q_{f}\right\|_{0, \Sigma}\left\|\Pi^{\mathfrak{S}} p^{\mathcal{T}}\right\|_{0, \Sigma}\right)
$$

The estimate (4.15) follows immediately using the trace Lemma 4.5 and the Poincaré inequality (Lem. 4.6).

Finally, applying (4.15) for $p^{D}=0, Q=0$ and $Q_{f}=0$, we find, using Poincaré lemma 4.6, that this implies $p^{\mathcal{T}}=0$. Since the discrete system is a square linear system we obtain existence and uniqueness of the solution for any data.

\subsection{Convergence of the numerical scheme}

\subsubsection{Compactness properties}

Lemma 4.9 (compactness lemma in $\Omega$ ). Let $\left(\mathcal{T}_{n}\right)_{n}$ be a family of meshes such that $\operatorname{size}\left(\mathcal{T}_{n}\right) \underset{n \rightarrow \infty}{\longrightarrow} 0$ and $\left(\operatorname{reg}\left(\mathcal{T}_{n}\right)\right)_{n}$ is bounded.

Suppose now given a family of discrete pressure fields $p^{\mathcal{T}_{n}}=\left(p^{\mathfrak{M}_{n}}, \gamma_{0, N} p^{\mathcal{T}_{n}}, \gamma^{ \pm} p^{\mathcal{T}_{n}}\right) \in E\left(\mathcal{T}_{n}\right)$ such that $\left(\left\|p^{\mathcal{T}_{n}}\right\|_{1, \mathcal{T}_{n}}\right)_{n}$ is bounded. Then there exists $p \in H^{1}(\Omega)$ with $\gamma_{0, D} p=p^{D}$ and such that, for a subsequence still denoted by $\left(p^{\mathcal{T}_{n}}\right)_{n}$, we have

$$
\begin{aligned}
& p^{\mathfrak{M}_{n}} \underset{n \rightarrow \infty}{\longrightarrow} \quad \text { strongly in } L^{2}(\Omega) \\
& \nabla^{\mathfrak{M}_{n}, p^{D}} p^{\mathcal{T}_{n}} \underset{n \rightarrow \infty}{\longrightarrow} \nabla p \text { weakly in }\left(L^{2}(\Omega)\right)^{2} \\
& \gamma^{ \pm} p^{\mathcal{T}_{n}} \underset{n \rightarrow \infty}{\longrightarrow} \gamma^{ \pm} p \text { weakly in } L^{2}(\Sigma) \\
& \llbracket p^{\mathcal{T}_{n}} \rrbracket \quad \underset{n \rightarrow \infty}{\longrightarrow} \llbracket p \rrbracket \text { weakly in } L^{2}(\Sigma) \\
& \gamma_{0, D} p^{\mathfrak{M}_{n}} \underset{n \rightarrow \infty}{\longrightarrow} p^{D} \text { strongly in } L^{2}\left(\Gamma_{D}\right) \\
& \gamma_{0, N} p^{\mathfrak{M}_{n}} \underset{n \rightarrow \infty}{\longrightarrow} \gamma_{0, N} p \text { weakly in } L^{2}\left(\Gamma_{N}\right) \text {. }
\end{aligned}
$$


Proof.

\section{- First step.}

We first prove that the family $\left(p^{\mathfrak{M}_{n}}\right)_{n}$ is relatively compact in $L^{2}(\Omega)$ using Kolmogoroff's theorem. To this end, we introduce, as in the classical theory, $\widetilde{p^{\mathfrak{M}_{n}}}$, the extension by 0 of $p^{\mathfrak{M}_{n}}$ on $\mathbb{R}^{2} \backslash \Omega$. It is clear that the assumption implies that $\left(p^{\mathfrak{M}_{n}}\right)_{n}$ is bounded in $L^{2}\left(\mathbb{R}^{2}\right)$.

We now prove, using methods similar to those in [16], that there exists $C>0$ such that for all $|\eta|<1$, we have $\left\|p^{\mathfrak{M}_{n}}(\cdot+\eta)-\widetilde{p^{\mathfrak{M}_{n}}}(\cdot)\right\|_{0, \mathbb{R}^{2}}^{2} \leq C|\eta|$. To this end, we define for all $\sigma \in \mathcal{E}$, the function $\chi_{\sigma}$ from $\mathbb{R}^{2} \times \mathbb{R}^{2}$ to $\{0,1\}$ by $\chi_{\sigma}(x, y)=1$ if $[x, y] \cap \sigma \neq \emptyset$ and $\chi_{\sigma}(x, y)=0$ otherwise. Denote by $c_{\sigma}=\left|\mathbf{n}_{\sigma} \cdot \frac{\eta}{|\eta|}\right|$ for any edge $\sigma$. Finally, notice that if $c_{\sigma}=0$ then $\chi_{\sigma}(x, x+\eta)=0$ for almost every $x \in \Omega$.

Hence, for almost every $x \in \Omega$ we have

$$
\begin{aligned}
\left|\widetilde{\mathfrak{M}_{n}}(x+\eta)-\widetilde{p^{\mathfrak{M}_{n}}}(x)\right| \leq & \sum_{\substack{\sigma \in \mathcal{E}_{\text {int }} \\
\sigma=\mathcal{K} \mid \mathcal{L}}} \chi_{\sigma}(x, x+\eta)\left|p_{\mathcal{K}}-p_{\mathcal{L}}\right|+\sum_{\sigma \in \mathcal{E}_{\text {ext }}} \chi_{\sigma}(x, x+\eta)\left|p_{\mathcal{K}_{\sigma}}\right| \\
& +\sum_{\sigma \in \mathfrak{S}} \chi_{\sigma}(x, x+\eta)\left(\left|p_{\mathcal{K}_{\sigma}^{+}}-p_{\sigma^{+}}\right|+\left|p_{\mathcal{K}_{\sigma}^{-}}-p_{\sigma^{-}}\right|+\left|p_{\sigma^{+}}-p_{\sigma^{-}}\right|\right) .
\end{aligned}
$$

Using the Cauchy-Schwarz inequality, it follows that

$$
\begin{aligned}
\left|\widetilde{\mathfrak{M}_{n}}(x+\eta)-\widetilde{p^{\mathfrak{M}_{n}}}(x)\right|^{2} \leq & C\left(\sum_{\substack{\sigma \in \mathcal{E}_{\text {int }} \\
\sigma=\mathcal{K} \mid \mathcal{L}}} \chi_{\sigma}(x, x+\eta) \frac{\left|p_{\mathcal{K}}-p_{\mathcal{L}}\right|^{2}}{c_{\sigma} d_{\sigma}}\right) \times\left(\sum_{\sigma \in \mathcal{E}_{\text {int }}} \chi_{\sigma}(x, x+\eta) c_{\sigma} d_{\sigma}\right) \\
& +C\left(\sum_{\sigma \in \mathcal{E}_{\text {ext }}} \chi_{\sigma}(x, x+\eta) \frac{\left|p_{\mathcal{K}_{\sigma}}\right|^{2}}{c_{\sigma}}\right) \times\left(\sum_{\sigma \in \mathcal{E}_{\text {ext }}} \chi_{\sigma}(x, x+\eta) c_{\sigma}\right) \\
& +C\left(\sum_{\sigma \in \mathfrak{S}} \chi_{\sigma}(x, x+\eta) \frac{\left|p_{\mathcal{K}_{\sigma}^{+}}-p_{\sigma^{+}}\right|^{2}}{c_{\sigma} d_{\mathcal{K}_{\sigma}^{+}}}\right) \times\left(\sum_{\sigma \in \mathfrak{S}} \chi_{\sigma}(x, x+\eta) c_{\sigma} d_{\mathcal{K}_{\sigma}^{+}}\right) \\
& +C\left(\sum_{\sigma \in \mathfrak{S}} \chi_{\sigma}(x, x+\eta) \frac{\left|p_{\mathcal{K}_{\sigma}^{-}}-p_{\sigma^{-}}\right|^{2}}{c_{\sigma} d_{\mathcal{K}_{\sigma}^{-}}}\right) \times\left(\sum_{\sigma \in \mathfrak{S}} \chi_{\sigma}(x, x+\eta) c_{\sigma} d_{\mathcal{K}_{\sigma}^{-}}\right) \\
& +C\left(\sum_{\sigma \in \mathfrak{S}} \chi_{\sigma}(x, x+\eta) \frac{\left|p_{\sigma^{+}}-p_{\sigma^{-}}\right|^{2}}{c_{\sigma} b_{f, \sigma}{ }^{2}}\right) \times\left(\sum_{\sigma \in \mathfrak{S}} \chi_{\sigma}(x, x+\eta) c_{\sigma} b_{f, \sigma}{ }^{2}\right) .
\end{aligned}
$$

It is proved in [16] that, there exists $C>0$ such that for any $x \in \Omega$ we have

$$
\sum_{\sigma \in \mathcal{E}_{\text {int }}} \chi_{\sigma}(x, x+\eta) c_{\sigma} d_{\sigma} \leq|\eta|+C \operatorname{size}\left(\mathcal{T}_{n}\right), \sum_{\sigma \in \mathcal{E}_{\text {ext }}} \chi_{\sigma}(x, x+\eta) c_{\sigma} d_{\sigma} \leq C .
$$

By exactly the same computations it follows that for any $x \in \Omega$ we have

$$
\sum_{\sigma \in \mathcal{E}_{\Sigma}} \chi_{\sigma}(x, x+\eta) d_{\mathcal{K}_{\sigma}^{+}, \sigma} c_{\sigma}+\sum_{\sigma \in \mathcal{E}_{\Sigma}} \chi_{\sigma}(x, x+\eta) d_{\mathcal{K}_{\sigma}^{-}, \sigma} c_{\sigma} \leq|\eta|+C \operatorname{size}\left(\mathcal{T}_{n}\right),
$$

and moreover, since we assumed here that $\Sigma$ is a segment (or even a finite number $N_{\Sigma}$ of connected straight lines) we have for any $x \in \Omega$

$$
\sum_{\sigma \in \mathcal{E}_{\Sigma}} \chi_{\sigma}(x, x+\eta) b_{f}^{2} c_{\sigma} \leq N_{\Sigma}\left\|b_{f}\right\|_{\infty, \Sigma}^{2}
$$


We use now these bounds in (4.22), then we integrate the inequality with respect to $x \in \Omega$. Noting that

$$
\int_{\mathbb{R}^{2}} \chi_{\sigma}(x, x+\eta) \mathrm{d} x \leq m(\sigma) c_{\sigma}|\eta|, \quad \forall \sigma, \forall \eta,
$$

we get, using in particular the trace Lemma 4.5, the estimate

$$
\begin{aligned}
\left\|\widetilde{p^{\mathfrak{M}_{n}}}(\cdot+\eta)-\widetilde{p^{\mathfrak{M}_{n}}}(\cdot)\right\|_{0, \mathbb{R}^{2}}^{2} \leq & C\left(|\eta|+\operatorname{size}\left(\mathcal{T}_{n}\right)\right)|\eta|\left(\sum_{\substack{\sigma \in \mathcal{E}_{\text {int }} \\
\sigma=\mathcal{K} \mid \mathcal{L}}} m\left(\mathcal{D}_{\sigma}\right)\left|\frac{p_{\mathcal{K}}-p_{\mathcal{L}}}{d_{\mathcal{K}, \mathcal{L}}}\right|^{2}\right) \\
& +C\left(|\eta|+\operatorname{size}\left(\mathcal{T}_{n}\right)\right)|\eta|\left(\sum_{\sigma \in \mathfrak{S}} m\left(\mathcal{D}_{\sigma^{ \pm}}\right)\left|\frac{p_{\mathcal{K}_{\sigma}^{ \pm}}-p_{\sigma^{ \pm}}}{d_{\mathcal{K}_{\sigma}^{ \pm}}}\right|^{2}\right) \\
& +C|\eta|\left(\sum_{\sigma \in \mathcal{E}_{\text {ext }}} m(\sigma)\left|p_{\mathcal{K}_{\sigma}}\right|^{2}\right)+C|\eta|\left(\sum_{\sigma \in \mathfrak{S}} m(\sigma)\left|\frac{p_{\sigma^{+}}-p_{\sigma^{-}}}{b_{f, \sigma}}\right|^{2}\right) \\
\leq & C|\eta|\left\|p^{\mathcal{T}_{n}}\right\|_{1, \mathcal{T}_{n}}^{2} \leq C^{\prime}|\eta| .
\end{aligned}
$$

Hence, by the Kolmogoroff's theorem, the sequence $\left(p^{\mathfrak{M}_{n}}\right)_{n}$ is compact, and then has a convergent subsequence in $L^{2}(\Omega)$. Let us denote by $p$ its limit.

- Second step. We have

$$
\begin{aligned}
\left\|\gamma_{0, D} p^{\mathfrak{M} n_{n}}-p^{D}\right\|_{0, \Gamma_{D}}^{2} & \leq 2 \sum_{\sigma \in \mathcal{E}_{\text {ext }}^{D}} m(\sigma)\left|p_{\mathcal{K}_{\sigma}}-p_{\sigma}^{D}\right|^{2}+2 \sum_{\sigma \in \mathcal{E}_{\text {ext }}^{D}} \int_{\sigma}\left|p^{D}(s)-p_{\sigma}^{D}\right|^{2} \mathrm{~d} s \\
& \leq C \sum_{\sigma \in \mathcal{E}_{\text {ext }}^{D}} d_{\mathcal{K}_{\sigma}, \sigma} m\left(\mathcal{D}_{\sigma}\right)\left|\frac{p_{\mathcal{K}_{\sigma}}-p_{\sigma}^{D}}{d_{\mathcal{K}_{\sigma}, \sigma}}\right|^{2}+C \sum_{\sigma \in \mathcal{E}_{\text {ext }}^{D}} m(\sigma) \int_{\sigma} \int_{\sigma}\left|\frac{p^{D}(s)-p^{D}(t)}{|s-t|}\right|^{2} \mathrm{~d} s \mathrm{~d} t \\
& \leq C \operatorname{size}\left(\mathcal{T}_{n}\right)\left(\left\|p^{\mathcal{T}_{n}}\right\|_{1, \mathcal{T}_{n}}^{2}+\left\|p^{D}\right\|_{\frac{1}{2}, \Gamma_{D}}^{2}\right) .
\end{aligned}
$$

Hence we obtain the strong $L^{2}$ convergence of the trace $\gamma_{0, D} p^{\mathfrak{M}_{n}}$ to the boundary data $p^{D}$.

- Third step. The bound $\left\|p^{\mathcal{T}_{n}}\right\|_{1, \mathcal{T}} \leq C$ and the trace Lemma 4.5 give that there exists $G \in\left(L^{2}(\Omega)\right)^{2}$, $q^{+} \in L^{2}(\Sigma), q^{-} \in L^{2}(\Sigma), q_{N} \in L^{2}\left(\Gamma_{N}\right), q_{D} \in L^{2}\left(\Gamma_{D}\right)$ such that, for a subsequence, we have

$$
\begin{array}{rll}
\nabla^{\mathfrak{M}_{n}, p^{D}} p^{\mathcal{T}_{n}} & & G \text { weakly in } L^{2}(\Omega), \\
\gamma^{ \pm} p^{\mathcal{T}_{n}} & \longrightarrow \infty & q^{ \pm} \text {weakly in } L^{2}(\Sigma) \\
\gamma_{0, N} p^{\mathfrak{M}_{n}} & &
\end{array}
$$

Let us now identify the functions $G, q^{ \pm}$and $q_{N}$. To this end, for any $\Phi \in \mathcal{S}^{2}(\mathcal{S}$ being defined in (3.2)), let us write

$$
\int_{\Omega} p^{\mathfrak{M}} \nabla \cdot \Phi \mathrm{d} x+\int_{\Omega} \nabla^{\mathfrak{M}, p^{D}} p^{\mathcal{T}} \cdot \Phi \mathrm{d} x=\sum_{\mathcal{K} \in \mathcal{T}} p_{\mathcal{K}} \sum_{\sigma \in \mathcal{E}_{\mathcal{K}}} \int_{\sigma} \Phi_{\mid \mathcal{K}} \cdot \mathbf{n}_{\mathcal{K}, \sigma} \mathrm{d} s+\sum_{\mathcal{D} \in \mathcal{D}} \nabla^{\mathfrak{M}, p^{D}} p^{\mathcal{T}} \cdot\left(\int_{\mathcal{D}} \Phi \mathrm{d} x\right)
$$

Noting that $\nabla^{\mathfrak{M}, p^{D}} p^{\mathcal{T}_{n}}$ is parallel to $\mathbf{n}_{\mathcal{K}, \sigma}$ on each diamond cell, it follows that

$$
\nabla^{\mathfrak{M}, p^{D}} p^{\mathcal{T}_{n}} \cdot\left(\int_{\mathcal{D}} \Phi \mathrm{d} x\right)=\nabla^{\mathfrak{M}, p^{D}} p^{\mathcal{T}_{n}} \cdot\left(\int_{\mathcal{D}} \Phi \cdot \mathbf{n}_{\mathcal{K}, \sigma}\right) \mathbf{n}_{\mathcal{K}, \sigma}
$$


Hence, reordering the first sum above as a sum over the diamond cells we derive that

$$
\begin{aligned}
\int_{\Omega} p^{\mathfrak{M}_{n}} \nabla \cdot \Phi \mathrm{d} x & +\int_{\Omega} \nabla^{\mathfrak{M}_{n}, p^{D}} p^{\mathcal{I}_{n}} \cdot \Phi \mathrm{d} x \\
& =\sum_{\mathcal{D} \in \mathcal{D}} m(\mathcal{D}) \nabla^{\mathfrak{M}_{n}, p^{D}} p^{\mathcal{T}_{n}} \cdot\left(\frac{1}{m(\mathcal{D})} \int_{\mathcal{D}} \Phi \cdot \mathbf{n}_{\mathcal{K}, \sigma} \mathrm{d} x-\frac{1}{m(\sigma)} \int_{\sigma} \Phi \cdot \mathbf{n}_{\mathcal{K}, \sigma} \mathrm{d} s\right) \mathbf{n}_{\mathcal{K}, \sigma} \\
& +\sum_{\sigma \in \mathcal{E}_{\text {ext }}^{D}} p_{\sigma}^{D} \int_{\sigma} \Phi \cdot \mathbf{n} \mathrm{d} s+\sum_{\sigma \in \mathcal{E}_{\text {ext }}^{N}} \int_{\sigma} \gamma_{0, N} p^{\mathfrak{M}_{n}} \Phi \cdot \mathbf{n} \mathrm{d} s \\
& +\sum_{\sigma \in \mathfrak{S}} \int_{\sigma}\left(\gamma^{+} p^{\mathcal{I}_{n}}\left(\gamma^{+} \Phi\right) \cdot \mathbf{n}^{+}+\gamma^{-} p^{\mathcal{T}_{n}}\left(\gamma^{-} \Phi\right) \cdot \mathbf{n}^{-}\right) \mathrm{d} s
\end{aligned}
$$

By using Taylor expansions, we easily see that for each diamond cell in the domain we have

$$
\left|\frac{1}{m(\mathcal{D})} \int_{\mathcal{D}} \Phi \mathrm{d} x-\frac{1}{m(\sigma)} \int_{\sigma} \Phi \mathrm{d} s\right| \leq C \operatorname{size}\left(\mathcal{T}_{n}\right)\|\Phi\|_{\mathcal{S}^{2}} .
$$

Note that this formula is still true, for $n$ large enough, for diamond cells touching the extremities of $\Sigma$ since $\Phi$ is assumed to be constant near $\partial \Sigma$, so that the left-hand side term in (4.24) is equal to zero for such diamond cells. It follows that the first term in the right-hand side of (4.23) tends to 0 when $n \rightarrow \infty$. Passing to the limit when $n$ goes to infinity in the other terms, it follows that

$$
\int_{\Omega} p \nabla \cdot \Phi \mathrm{d} x+\int_{\Omega} G \cdot \Phi \mathrm{d} x=\int_{\Gamma_{D}} p^{D} \Phi \cdot \mathbf{n} \mathrm{d} s+\int_{\Gamma_{N}} q_{N} \Phi \cdot \mathbf{n} \mathrm{d} s+\int_{\Sigma}\left(q^{+}\left(\gamma^{+} \Phi\right) \cdot \mathbf{n}^{+}+q^{-}\left(\gamma^{-} \Phi\right) \cdot \mathbf{n}^{-}\right) \mathrm{d} s .
$$

This formula holds for any $\Phi \in \mathcal{S}^{2}$, and then for any $\Phi \in H^{1}(\Omega)^{2}$ thanks to Proposition 3.3. We conclude that $p \in H^{1}(\Omega)$ and $G=\nabla p, \gamma_{0, D} p=p^{D}, q_{N}=\gamma_{0, N} p, q^{ \pm}=\gamma^{ \pm} p$ and the claim is proved.

We can also obtain by similar and simpler arguments a $1 \mathrm{D}$ compactness result on the fracture $\Sigma$ that we state here.

Lemma 4.10 (compactness lemma in $\Sigma$ ). Let $\left(\mathcal{T}_{n}\right)_{n}$ be a family of meshes such that $\operatorname{size}\left(\mathcal{T}_{n}\right) \underset{n \rightarrow \infty}{\longrightarrow} 0$ and $\left(\operatorname{reg}\left(\mathcal{T}_{n}\right)\right)_{n}$ is bounded. Let now $p^{\mathfrak{S}_{n}} \in E\left(\mathfrak{S}_{n}\right)$ be a family of fracture pressure fields such that $\left(\left\|p^{\mathfrak{S}_{n} \rightarrow \infty}\right\|_{1, \mathfrak{S}_{n}}\right)_{n}$ is bounded. Then, there exists $p_{f} \in H^{1}(\Sigma)$ such that, for a subsequence still denoted by $p^{\mathfrak{S}_{n}}$, we have

$$
\begin{array}{rlr}
p^{\mathfrak{S}_{n}} & & \\
\nabla^{\mathfrak{S}_{n}} p^{\mathfrak{S}_{n}} & \underset{n \rightarrow \infty}{\longrightarrow} & p_{f} \text { strongly in } L^{2}(\Sigma), \\
& \nabla_{\boldsymbol{\tau}} p_{f} \text { weakly in } L^{2}(\Sigma) .
\end{array}
$$

\subsubsection{Convergence theorem}

We are now in position to prove the main result of this paper, which shows the convergence of the finite volume scheme to a solution of our asymptotic model of flows in fractured porous media.

This result does not give an error estimate since it would require regularity results for the solution of the asymptotic problem (3.5)-(3.10). This problem should be addressed in a further work. Nevertheless, the scheme under study is expected to be first order convergent in the discrete $H^{1}$ norms, like in the classical situation of the Darcy problem in a non-fractured porous medium (see [16]).

Theorem 4.11. Let $\left(\mathcal{T}_{n}\right)_{n}$ be a family of meshes such that $\operatorname{size}\left(\mathcal{T}_{n}\right) \underset{n \rightarrow \infty}{\longrightarrow} 0$ and $\left(\operatorname{reg}\left(\mathcal{I}_{n}\right)\right)_{n}$ is bounded. Then, the unique solution $p^{\mathcal{I}_{n}}$ to the scheme (4.4)-(4.11) for the mesh $\mathcal{T}_{n}$ converges to the unique solution $p \in \mathcal{W}$ of problem (3.5)-(3.10). 
More precisely, $\left(p^{\mathfrak{M}_{n}}\right)_{n}$ converges strongly to $p$ in $L^{2}(\Omega),\left(\nabla^{\mathfrak{M}_{n}, p^{D}} p^{\mathfrak{M}_{n}}\right)_{n}$ converges weakly to $\nabla p$ in $\left(L^{2}(\Omega)\right)^{2}$, $\left(\Pi^{\mathfrak{S}_{n}} p^{\mathcal{T}_{n}}\right)_{n}$ converges strongly to $\Pi_{f} p$ in $L^{2}(\Sigma)$ and $\left(\nabla^{\mathfrak{S}_{n}} \Pi^{\mathfrak{S}_{n}} p^{\mathcal{T}_{n}}\right)_{n}$ converges weakly to $\nabla^{\mathfrak{S}} \Pi_{f} p$ in $L^{2}(\Sigma)$.

Proof. Notice that we are going to show the convergence of a subsequence which is enough to prove the claim since we already know that the solution to (3.5)-(3.10) is unique.

- From the a priori estimate obtained in Lemma 4.8, and the compactness Lemmas 4.9 and 4.10, we obtain that there exists $p \in H^{1}(\Omega)$ and $p_{f} \in H^{1}(\Sigma)$ such that (4.16)-(4.21) hold and moreover

$$
\begin{array}{rll}
\Pi^{\mathfrak{S}_{n}} p^{\mathcal{I}_{n}} & & \\
\nabla_{n \rightarrow \infty} & p_{f} \text { strongly in } L^{2}(\Sigma) \\
\nabla^{\mathfrak{S}_{n}} \Pi^{\mathfrak{S}_{n}} p^{\mathcal{I}_{n}} & \underset{n \rightarrow \infty}{\longrightarrow} & \nabla_{\tau} p_{f} \text { weakly in } L^{2}(\Sigma) .
\end{array}
$$

We want now to show that $p_{f}=\Pi_{f} p$, that $p \in \mathcal{W}$ and that it is a solution to (3.5)-(3.10).

- From equations (4.11) and (4.19) we derive that

$$
\overline{\mathbf{v}^{p^{T_{n}}} \cdot \boldsymbol{\nu}} \underset{n \rightarrow+\infty}{\longrightarrow}-\frac{\mathbf{K}_{f, \boldsymbol{\nu}}}{\mu}\left(\frac{\llbracket p \rrbracket}{b_{f}}\right) \text { weakly in } L^{2}(\Sigma) .
$$

From now on, we need to perform a separate analysis for the two cases $\xi>\frac{1}{2}$ and $\xi=\frac{1}{2}$, since in the first case we have an additional a priori estimate and we have to pass to the limit, as $n$ goes to infinity, independently in the problem in the porous matrix and in the fracture, whereas in the second case we must treat simultaneously the two equations.

- The case $\xi>\frac{1}{2}$

- Thanks to the a priori estimate (4.15) and definition (4.3) of the norm $\|\cdot\|_{W^{\mathcal{T}}, p^{D}}$, we see that for $\xi>\frac{1}{2}$, the quantity $\llbracket \mathbf{v}^{p^{T_{n}}} \cdot \boldsymbol{\nu} \rrbracket$ is bounded in $L^{2}(\Sigma)$. Hence, there exists $\Psi \in L^{2}(\Sigma)$ such that, for a subsequence,

$$
\llbracket \mathbf{v}^{p^{T_{n}}} \cdot \boldsymbol{\nu} \rrbracket \underset{n \rightarrow \infty}{\longrightarrow} \Psi \text { weakly in } L^{2}(\Sigma) .
$$

Notice that, thanks to assumptions (2.2), it is easily seen that $\sum_{\sigma \in \mathfrak{S}_{n}} \mathbb{1}_{\sigma} b_{f, \sigma}$ converges strongly to $b_{f}$ in $L^{2}(\Sigma)$ for instance. Hence, we can take the limit as $n$ goes to infinity in equation (4.8) and find

$$
p_{f}=\frac{1}{2}\left(\gamma^{+} p+\gamma^{-} p\right)+\frac{(2 \xi-1) \mu}{4 \mathbf{K}_{f, \nu}} b_{f} \Psi .
$$

- Let now $\phi_{f}$ be a function in $\mathcal{C}^{2}(\Sigma)$, which is constant near the two ends $\partial \Sigma^{+}$and $\partial \Sigma^{-}$of $\Sigma$. Let us take $\phi^{\mathfrak{S}}=\left(\left(\phi_{f}\left(x_{\sigma}\right)\right)_{\sigma}, \phi_{f}\left(\partial \Sigma^{-}\right), \phi_{f}\left(\partial \Sigma^{+}\right)\right) \in E(\mathfrak{S})$ in (4.13). It follows that, for $n$ large enough (so that $\phi_{f}$ is constant on the two edges $\sigma$ touching the boundary of $\Sigma$ ) we have

$$
\begin{aligned}
\int_{\Sigma} \frac{\mathbf{K}_{f, \boldsymbol{\tau}}}{\mu}\left(\nabla^{\mathfrak{S}_{n}} \Pi^{\mathfrak{S}_{n}} p^{\mathcal{T}_{n}}\right)\left(\nabla_{\boldsymbol{\tau}} \phi_{f}(s)+R_{n}(s)\right) b_{f}(s) \mathrm{d} s & =\int_{\Sigma} Q_{f}(s)\left(\phi_{f}(s)+r_{n}(s)\right) b_{f}(s) \mathrm{d} s \\
& -\int_{\Sigma} \llbracket \mathbf{v}^{p^{\mathcal{T}_{n}}} \cdot \boldsymbol{\nu} \rrbracket\left(\phi_{f}(s)+r_{n}(s)\right) \mathrm{d} s,
\end{aligned}
$$

where

$$
\begin{gathered}
R_{n}(s)=\frac{\phi_{f}\left(x_{\sigma^{\prime}}\right)-\phi_{f}\left(x_{\sigma}\right)}{d_{\sigma, \sigma^{\prime}}}\left(\boldsymbol{\tau}_{\sigma, \sigma^{\prime}} \cdot \boldsymbol{\tau}\right)-\nabla_{\boldsymbol{\tau}} \phi_{f}(s), \quad \forall s \in S_{e}, \quad \forall e=\sigma \mid \sigma^{\prime} \in \mathfrak{V}_{\mathrm{int}}, \\
R_{n}(s)=0, \quad \forall s \in S_{e}, \quad \forall e \in\left\{\partial \Sigma^{-}, \partial \Sigma^{+}\right\}
\end{gathered}
$$

and

$$
r_{n}(s)=\phi_{f}\left(x_{\sigma}\right)-\phi_{f}(s), \quad \forall s \in \sigma, \quad \forall \sigma \in \mathfrak{S}_{n}
$$


Since $\phi_{f}$ is smooth, we easily see that $\left\|R_{n}\right\|_{\infty, \Sigma}+\left\|r_{n}\right\|_{\infty, \Sigma} \leq \operatorname{size}\left(\mathcal{T}_{n}\right)\left\|\phi_{f}\right\|_{\mathcal{C}^{2}}$. Hence, we can pass to the limit as $n$ goes to infinity in (4.29) by using (4.26) and (4.27). We find that $p_{f}$ satisfies

$$
-\nabla_{\boldsymbol{\tau}}\left(b_{f}(s) \frac{\mathbf{K}_{f, \boldsymbol{\tau}}}{\mu}\left(\nabla_{\boldsymbol{\tau}} p_{f}\right)\right)=b_{f} Q_{f}-\Psi \text { on } \Sigma,
$$

as well as the Neumann boundary condition on $\partial \Sigma$.

- Let $\phi \in \mathcal{S}$ (see definition (3.2)) such that $\phi=0$ on $\Gamma_{D}$. We use here the center-valued projection $\Pi^{\mathcal{T}_{n}, c} \phi=\left(\Pi^{\mathfrak{M}_{n}, c} \phi, \gamma_{0, N} \Pi^{\mathcal{T}_{n}, c} \phi, \gamma^{ \pm} \Pi^{\mathcal{T}_{n}, c} \phi\right)$ of $\phi$ on the mesh $\mathcal{T}_{n}$ defined by

$$
\left\{\begin{aligned}
\Pi^{\mathfrak{M}_{n}, c} \phi & =\left(\phi_{\mathcal{K}}\right)_{\mathcal{K} \in \mathfrak{M}_{n}}, \phi_{\mathcal{K}}=\phi\left(x_{\mathcal{K}}\right), \\
\gamma^{ \pm} \Pi^{\mathcal{T}_{n}, c} \phi & =\left(\phi_{\sigma^{ \pm}}\right)_{\sigma \in \mathfrak{S}_{n}}, \phi_{\sigma^{ \pm}}=\gamma^{ \pm} \phi\left(x_{\sigma}\right), \\
\gamma_{0, N} \Pi^{\mathcal{T}_{n}, c} \phi & =\left(\phi_{\sigma}^{N}\right)_{\sigma \in \mathcal{E}_{\text {ext }}^{N}}, \phi_{\sigma}^{N}=\gamma_{0, N} \phi\left(x_{\sigma}\right) .
\end{aligned}\right.
$$

It follows from (4.12) that

$$
\begin{gathered}
\sum_{\mathcal{D} \in \mathcal{D}} \int_{\mathcal{D}} \frac{\mathbf{K}_{m}}{\mu}\left(\nabla^{\mathfrak{M}_{n}, p^{D}} p^{\mathcal{T}_{n}}\right) \cdot\left(\left(\nabla \phi(x) \cdot \mathbf{n}_{\sigma}+\widetilde{R}_{n}(x)\right) \mathbf{n}_{\sigma}\right) \mathrm{d} x-\int_{\Sigma}\left(\bar{\phi}+\frac{r_{n}^{+}+r_{n}^{-}}{2}\right) \llbracket \mathbf{v}^{p^{T_{n}}} \cdot \boldsymbol{\nu} \rrbracket \mathrm{d} s \\
+\int_{\Sigma} \frac{\mathbf{K}_{f, \boldsymbol{\nu}}}{\mu} \frac{\llbracket p^{\mathcal{T}_{n}} \rrbracket}{b_{f, \sigma}}\left(\frac{\llbracket \phi \rrbracket+r_{n}^{+}-r_{n}^{-}}{b_{f, \sigma}}\right) b_{f} \mathrm{~d} s=\int_{\Omega} Q(x) \Pi^{\mathfrak{M}_{n}, c} \phi \mathrm{d} x,
\end{gathered}
$$

where

$$
\begin{gathered}
\widetilde{R}_{n}(x)=\nabla \phi(x) \cdot \mathbf{n}_{\sigma}-\nabla^{\mathfrak{M}_{n}, 0} \Pi^{\mathcal{T}_{n}, c} \phi \cdot \mathbf{n}_{\sigma}, \quad \forall x \in \mathcal{D}_{\sigma}, \quad \forall \mathcal{D}_{\sigma} \in \mathfrak{D}, \\
r_{n}^{ \pm}(s)=\gamma^{ \pm} \phi\left(x_{\sigma}\right)-\gamma^{ \pm} \phi(s), \quad \forall s \in \sigma \in \mathfrak{S}_{n} .
\end{gathered}
$$

Since $\phi$ is smooth and vanishes on $\Gamma_{D}$, we easily get that

$$
\left\|\widetilde{R}_{n}\right\|_{\infty, \Omega}+\left\|r_{n}^{ \pm}\right\|_{\infty, \Sigma} \leq C \operatorname{size}\left(\mathcal{T}_{n}\right)\|\phi\|_{\mathcal{S}} .
$$

Furthermore, on each diamond cell the vector $\nabla^{\mathfrak{M}_{n}, p^{D}} p^{\mathcal{T}_{n}}$ is parallel to $\mathbf{n}_{\sigma}$. Hence we get

$$
\int_{\mathcal{D}} \frac{\mathbf{K}_{m}}{\mu} \nabla^{\mathfrak{M}_{n}, p^{D}} p^{\mathcal{T}_{n}} \cdot\left(\left(\nabla \phi(x) \cdot \mathbf{n}_{\sigma}\right) \mathbf{n}_{\sigma}\right) \mathrm{d} x=\int_{\mathcal{D}} \frac{\mathbf{K}_{m}}{\mu} \nabla^{\mathfrak{M}_{n}, p^{D}} p^{\mathcal{T}_{n}} \cdot \nabla \phi(x) \mathrm{d} x,
$$

so that finally, we can pass to the limit when $n$ goes to infinity in (4.31) and obtain

$$
\int_{\Omega} \frac{\mathbf{K}_{m}}{\mu} \nabla p \cdot \nabla \phi \mathrm{d} x+\int_{\Sigma} \frac{\mathbf{K}_{f, \nu}}{\mu}\left(\frac{\llbracket p \rrbracket}{b_{f}}\right) \frac{\llbracket \phi \rrbracket}{b_{f}} b_{f} \mathrm{~d} s-\int_{\Sigma} \bar{\phi} \Psi \mathrm{d} s=\int_{\Omega} Q \phi \mathrm{d} x .
$$

Taking $\phi \in \mathcal{C}_{c}^{\infty}(\Omega)$ in this formulation, we first get that $p$ satisfies

$$
\nabla \cdot \mathbf{v}^{p}=Q \text { in } \Omega
$$

with $\mathbf{v}^{p}=-\frac{\mathbf{K}_{m}}{\mu}(\nabla p)$ and thus $\mathbf{v}^{p} \in H_{\mathrm{div}}(\Omega)$.

Since $\mathcal{S}$ is dense in $H^{1}(\Omega)$ (Prop. 3.3), we get that (4.32) holds for any $\phi \in H^{1}(\Omega)$ so that, comparing to the Stokes formula (3.1), we finally obtain

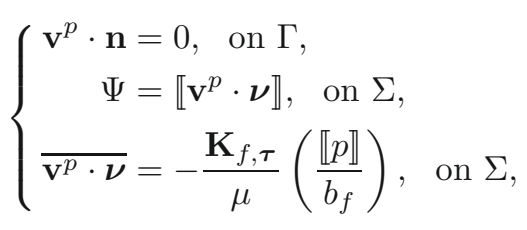


and then by (4.28), we find that the fracture pressure satisfies

$$
p_{f}=\bar{p}-\frac{(2 \xi-1) \mu}{4 \mathbf{K}_{f, \boldsymbol{\nu}}} b_{f} \llbracket \mathbf{v}^{p} \cdot \boldsymbol{\nu} \rrbracket,
$$

that is $p_{f}=\Pi_{f} p$. Finally, replacing the above value for $\Psi$ in (4.30), we recover the Darcy equation in the fracture and the claim is proved.

\section{- The case $\xi=\frac{1}{2}$}

Note that, in that case, we can easily pass to the limit when $n$ goes to infinity in (4.8) to find that

$$
p_{f}=\frac{1}{2}\left(\gamma^{+} p+\gamma^{-} p\right)=\Pi_{f} p
$$

Unfortunately we do not have now any a priori bound on $\llbracket \mathbf{v}^{p^{\tau_{n}}} \cdot \boldsymbol{\nu} \rrbracket$. Hence, we are not able to pass to the limit independently in (4.12) and (4.13) as $n$ goes to infinity as before. That is the reason why we are going to treat simultaneously the continuity equation in the porous matrix and in the fracture by passing to the limit in the global formulation (4.14) (the last term being 0 since $\xi=\frac{1}{2}$ ).

Taking here also $\phi^{\mathcal{T}_{n}}=\Pi^{\mathcal{T}_{n}, c} \phi$ for any $\phi \in \mathcal{S}$ in this formula, we get

$$
\begin{aligned}
& \sum_{\mathcal{D} \in \mathfrak{D}} \int_{\mathcal{D}} \frac{\mathbf{K}_{m}}{\mu}\left(\nabla^{\mathfrak{M}_{n}, p^{D}} p^{\mathcal{T}_{n}}\right) \cdot\left(\left(\nabla \phi(x) \cdot \mathbf{n}_{\sigma}+\widetilde{R}_{n}(x)\right) \mathbf{n}_{\sigma}\right) \mathrm{d} x+ \\
& \int_{\Sigma} \frac{\mathbf{K}_{f, \boldsymbol{\nu}}}{\mu}\left(\frac{\llbracket p^{\mathcal{T}_{n}} \rrbracket}{b_{f, \sigma}}\right)\left(\frac{\llbracket \phi \rrbracket+r_{n}^{+}-r_{n}^{-}}{b_{f, \sigma}}\right) b_{f} \mathrm{~d} s+\int_{\Sigma} \frac{\mathbf{K}_{f, \boldsymbol{\tau}}}{\mu}\left(\nabla^{\mathfrak{S}_{n}} \Pi^{\mathfrak{S}_{n}} p^{\mathcal{T}_{n}}\right)\left(\nabla_{\boldsymbol{\tau}} \bar{\phi}(s)+R_{n}(s)\right) b_{f}(s) \mathrm{d} s= \\
& \int_{\Omega} Q(x) \Pi^{\mathfrak{M}_{n}, c} \phi \mathrm{d} x+\int_{\Sigma} Q_{f}(s)\left(\bar{\phi}(s)+r_{n}(s)\right) b_{f}(s) \mathrm{d} s
\end{aligned}
$$

By the same arguments as that used above, we can pass to the limit when $n$ goes to infinity in this formula and obtain

$$
\begin{aligned}
\int_{\Omega} \frac{\mathbf{K}_{m}}{\mu} \nabla p \cdot \nabla \phi \mathrm{d} x & +\int_{\Sigma} \frac{\mathbf{K}_{f, \boldsymbol{\tau}}}{\mu}\left(\nabla_{\boldsymbol{\tau}} \Pi_{f} p\right) \nabla_{\boldsymbol{\tau}} \bar{\phi}(s) b_{f}(s) \mathrm{d} s+\int_{\Sigma} \frac{\mathbf{K}_{f, \boldsymbol{\nu}}}{\mu}\left(\frac{\llbracket p \rrbracket}{b_{f}}\right) \frac{\llbracket \phi \rrbracket}{b_{f}} b_{f} \mathrm{~d} s \\
& =\int_{\Omega} Q \phi \mathrm{d} x+\int_{\Sigma} Q_{f} \bar{\phi} b_{f} \mathrm{~d} s .
\end{aligned}
$$

Taking first $\phi \in \mathcal{C}^{\infty}(\bar{\Omega})$ vanishing in a neighborhood of the fracture $\Sigma$, we see that $\nabla \cdot \mathbf{v}^{p}=Q$ in $\Omega$ and that the Neumann boundary condition (3.7) is satisfied.

Furthermore, we know from the compactness Lemma 4.10 that $\Pi_{f} p \in H^{1}(\Sigma)$, that is to say $\Pi_{f} p=\bar{p} \in H^{1}(\Sigma)$ by (4.33). It follows that $p$ lies in the space $\mathcal{W}$ defined in Section 3.1.2.

We use now the Stokes formula (3.1) for $\mathbf{v}^{p}$ and any $\phi \in \mathcal{S}$ and we compare to (4.34). It follows that

$$
\begin{aligned}
\left\langle\llbracket \mathbf{v}^{p} \cdot \boldsymbol{\nu} \rrbracket, \bar{\phi}\right\rangle_{H^{-\frac{1}{2}}(\Sigma), H^{\frac{1}{2}}(\Sigma)} & +\left\langle\overline{\mathbf{v}^{p} \cdot \boldsymbol{\nu}}, \llbracket \phi \rrbracket\right\rangle \\
& +\int_{\Sigma} \frac{\mathbf{K}_{f, \boldsymbol{\tau}}}{\mu}\left(\nabla_{\boldsymbol{\tau}} \bar{p}\right) \nabla_{\boldsymbol{\tau}} \bar{\phi} b_{f}(s) \mathrm{d} s+\int_{\Sigma} \frac{\mathbf{K}_{f, \boldsymbol{\nu}}}{\mu}\left(\frac{\llbracket p \rrbracket}{b_{f}}\right) \frac{\llbracket \phi \rrbracket}{b_{f}} b_{f} \mathrm{~d} s=\int_{\Sigma} Q_{f} \bar{\phi} b_{f} \mathrm{~d} s .
\end{aligned}
$$

- For any $\psi \in \mathcal{C}_{c}^{\infty}(\Sigma)$, we easily build a function $\phi \in \mathcal{S}$ such that $\gamma^{+} \phi=\psi / 2$ and $\gamma^{-} \phi=-\psi / 2$, so that $\bar{\phi}=0$ and $\llbracket \phi \rrbracket=\psi$. Using this particular test function in (4.35) for any such $\psi$, we deduce that

$$
\overline{\mathbf{v}^{p} \cdot \boldsymbol{\nu}}=-\frac{\mathbf{K}_{f, \boldsymbol{\nu}}}{\mu}\left(\frac{\llbracket p \rrbracket}{b_{f}}\right) .
$$


- Consider now the space $\mathcal{S}_{\Sigma}=\left\{\psi \in \mathcal{C}^{\infty}(\Sigma), \psi\right.$ is constant near $\partial \Sigma^{-}$and $\left.\partial \Sigma^{+}\right\}$. For any $\psi \in \mathcal{S}_{\Sigma}$, we can easily build $\phi \in \mathcal{S}$ such that $\llbracket \phi \rrbracket=0$ and $\bar{\phi}=\psi$, so that we get

$$
\left\langle\llbracket \mathbf{v}^{p} \cdot \boldsymbol{\nu} \rrbracket, \psi\right\rangle_{H^{-\frac{1}{2}}(\Sigma), H^{\frac{1}{2}}(\Sigma)}+\int_{\Sigma} \frac{\mathbf{K}_{f, \boldsymbol{\tau}}}{\mu}\left(\nabla_{\boldsymbol{\tau}} \bar{p}\right) \nabla_{\boldsymbol{\tau}} \psi b_{f}(s) \mathrm{d} s=\int_{\Sigma} Q_{f} \psi b_{f} \mathrm{~d} s .
$$

The set $\mathcal{S}_{\Sigma}$ being dense in $H^{1}(\Sigma)$, we finally find that the continuity equation (3.8) and the boundary condition (3.9) are satisfied in the fracture $\Sigma$.

\section{- Conclusion}

We proved the existence of a solution of the problem (3.5)-(3.9) for all $\xi \geq \frac{1}{2}$. Furthermore, this problem admits at most one solution as shown in the previous section. Hence, we conclude that the whole sequence $\left(p^{\mathcal{T}_{n}}\right)_{n}$ converges to the unique solution of problem (3.5)-(3.9) in the sense given in the statement of the theorem.

\section{NumericAl RESUlts FOR SOME PARTIALly AND FULLY IMMERSED FRACTURES}

We present numerical results for a computational domain $\Omega=[0,1]^{2}$. Let us recall that the permeability tensor is isotropic in the porous matrix - we choose $\mathbf{K}_{m}=\mathrm{Id}$ - and diagonal in the curvilinear frame of the fractures. We have taken in this section $\mu=1$ and $Q=0, Q_{f}=0$, so that the flow is only generated by the boundary conditions.

We always take the quadrature parameter $\xi$ equal to $\frac{1}{2}$, except in Section 5.3 where the influence of this parameter is investigated.

We first describe in Section 5.1, in a few words, the way we obtained the reference solutions that we will use in order to discuss the validity of the asymptotic models under study. All the numerical results for both the asymptotic and the global Darcy models are presented when the mesh convergence is reached, that is when the magnitude of the difference between the two solutions do not vary when the meshes are refined. We represent the distribution of the pressure field inside the domain from dark (high pressure values) to light (low pressure values). We also represent some of the streamlines of each flow.

\subsection{Computing reference solutions of the global model $\left(\mathcal{P}_{\mathcal{G}}\right)$}

The difficulties in approximating by finite volume methods the two-permeabilities global Darcy model $\left(\mathcal{P}_{\mathcal{G}}\right)$ are twofold. The first one comes from the very different scales in the flow (the small aperture of the fractures, the high permeability ratios, ...). The second one comes from the anisotropy of the equation inside the fracture which requires a "gradient reconstruction" method to approximate fluxes across the edges. To this end, we choose a Discrete Duality Finite Volume approach (see $[2,14]$ ) which consists in approximating the solution simultaneously on a primal and a dual mesh. In the presence of strong discontinuities of the permeability tensor, these classical DDFV schemes still converge but slowly (at rate 1/2). To recover a first order scheme, a modified DDFV scheme is proposed and analysed in [11,20]. This m-DDFV scheme is proved to be of first order in the discrete $H^{1}$-norm, even in the presence of high permeability jumps.

\subsection{Results for a straight half-fracture with constant aperture}

We consider the half fracture $\Sigma \times\left[-b_{f} / 2, b_{f} / 2\right]$, with $\Sigma=\{(x, y)$ such that $x=0.5, y \geq 0.5\}$ and $b_{f}$ is constant and equal to 0.01 . Let us describe the various configurations and boundary conditions we propose to illustrate various typical flows and the behavior of the model under study in each case. We use in this section rectangular meshes for both global model (65 536 control volumes) and asymptotic model (16 364 control volumes), in order to obtain a precise comparison of the two models along the line $y=0.75$. 


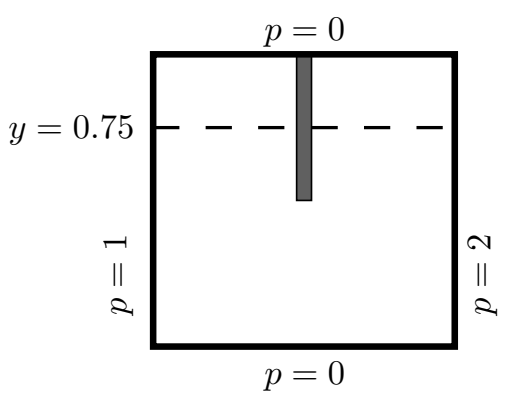

Fully permeable case $\mathbf{K}_{f, \boldsymbol{\tau}}=10^{6}, \mathbf{K}_{f, \boldsymbol{\nu}}=10^{2}$

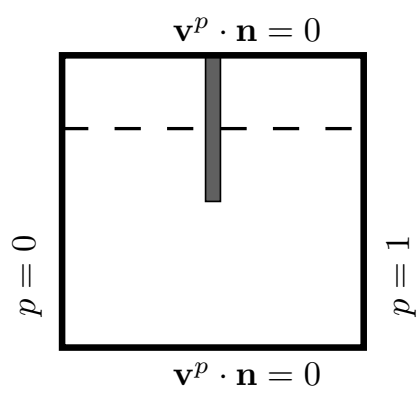

Impermeable case

$\mathbf{K}_{f, \boldsymbol{\tau}}=\mathbf{K}_{f, \boldsymbol{\nu}}=10^{-7}$

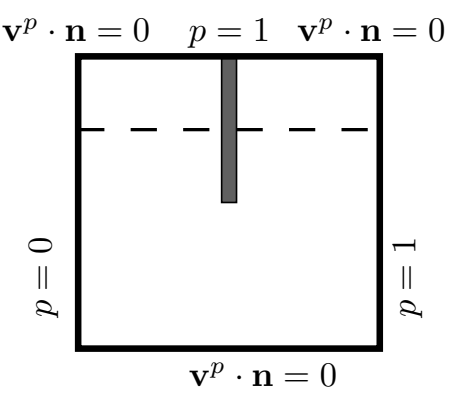

Partially permeable case

$\mathbf{K}_{f, \boldsymbol{\tau}}=100, \mathbf{K}_{f, \boldsymbol{\nu}}=100$

Figure 4. Configurations and boundary conditions for a straight half fracture.

- Highly permeable fracture, see Figure 4 (left).

The quantities $b_{f} \mathbf{K}_{f, \boldsymbol{\tau}}$ and $\frac{\mathbf{K}_{f, \boldsymbol{\nu}}}{b_{f}}$ are of the same order e.g. $\mathbf{K}_{f, \boldsymbol{\tau}}=10^{6}, \mathbf{K}_{f, \boldsymbol{\nu}}=100$. We impose Dirichlet boundary conditions on $\partial \Omega: p(\cdot, 0)=0, p(\cdot, 1)=0, p(0, \cdot)=1, p(1, \cdot)=2$.

- Impermeable fracture, see Figure 4 (center).

The quantity $b_{f} \mathbf{K}_{f, \boldsymbol{\tau}}$ is close to zero and $\frac{\mathbf{K}_{f, \boldsymbol{\nu}}}{b_{f}}$ remains small e.g. $\mathbf{K}_{f, \boldsymbol{\tau}}=10^{-7}, \mathbf{K}_{f, \boldsymbol{\nu}}=10^{-7}$. We impose mixed boundary conditions on $\partial \Omega: \mathbf{v}^{p} \cdot \mathbf{n}(\cdot, 0)=0, \mathbf{v}^{p} \cdot \mathbf{n}(\cdot, 1)=0, p(0, y)=0, p(1, \cdot)=1$.

- Fracture with intermediate properties, see Figure 4 (right).

In this test, previously performed for the corresponding non-immersed fracture in [21,22], the ratio between $\mathbf{K}_{f, \boldsymbol{\nu}} / b_{f}$ and $\mathbf{K}_{m}$ is high whereas $b_{f} \mathbf{K}_{f, \boldsymbol{\tau}}$ and $\mathbf{K}_{m}$ are of same order e.g. $\mathbf{K}_{f, \boldsymbol{\tau}}=100$, $\mathbf{K}_{f, \boldsymbol{\nu}}=100$.

We impose mixed boundary conditions on $\partial \Omega: p(0, \cdot)=0, p(1, \cdot)=1, \mathbf{v}^{p} \cdot \mathbf{n}(\cdot, 0)=0, \mathbf{v}^{p} \cdot \mathbf{n}(x, 1)=0$ for any $x \neq \frac{1}{2}$, and $p\left(\frac{1}{2}, 1\right)=1$.

In Figure 5, the results obtained by the resolution of the global Darcy two-permeabilities model $\left(\mathcal{P}_{\mathcal{G}}\right)$ by the m-DDFV methods and the resolution of the asymptotic model $\left(\mathcal{P}_{\mathcal{A}}\right)$ are compared. At the top part of the figure, we show the pressure field and some streamlines indicating the shape of the flow we are looking at in each case. In the middle part of the figure, we show the pressure on the horizontal cutline $y=0.75$ whereas in the bottom part we show the pressure field along the fracture. In any case, the curves for the global Darcy model and the asymptotic models are nearly identical.

These results show the ability of the asymptotic model to simulate efficiently all kinds of flows in fractured porous media, from impermeable fractures (where the pressure is not continuous across the fracture) to highly permeable ones (where the mass flux is not continuous across the fracture). The gain in using the asymptotic model is especially obvious for large permeability ratios or very anisotropic fracture permeabilities: the resolution of the global Darcy model typically requires six times more degrees of freedom than the asymptotic one in order to achieve mesh convergence of the numerical solutions for a given tolerance.

\subsection{Influence of the quadrature parameter $\xi$}

In many situations, we observed that the influence of the quadrature parameter $\xi$ is almost negligible and any value in the interval $[1 / 2,1]$ gives satisfactory results. Nevertheless, the influence of the parameter $\xi$ can be observed in some cases. We choose here for instance a fracture with a very high anisotropy $\mathbf{K}_{f, \boldsymbol{\tau}}=100$, $\mathbf{K}_{f, \boldsymbol{\nu}}=10^{-4}$. We consider two different Dirichlet boundary conditions for the pressure (see Fig. 6), the first one leading to a symmetric flow, and the second one leading to a non-symmetric flow. 
Permeable fracture
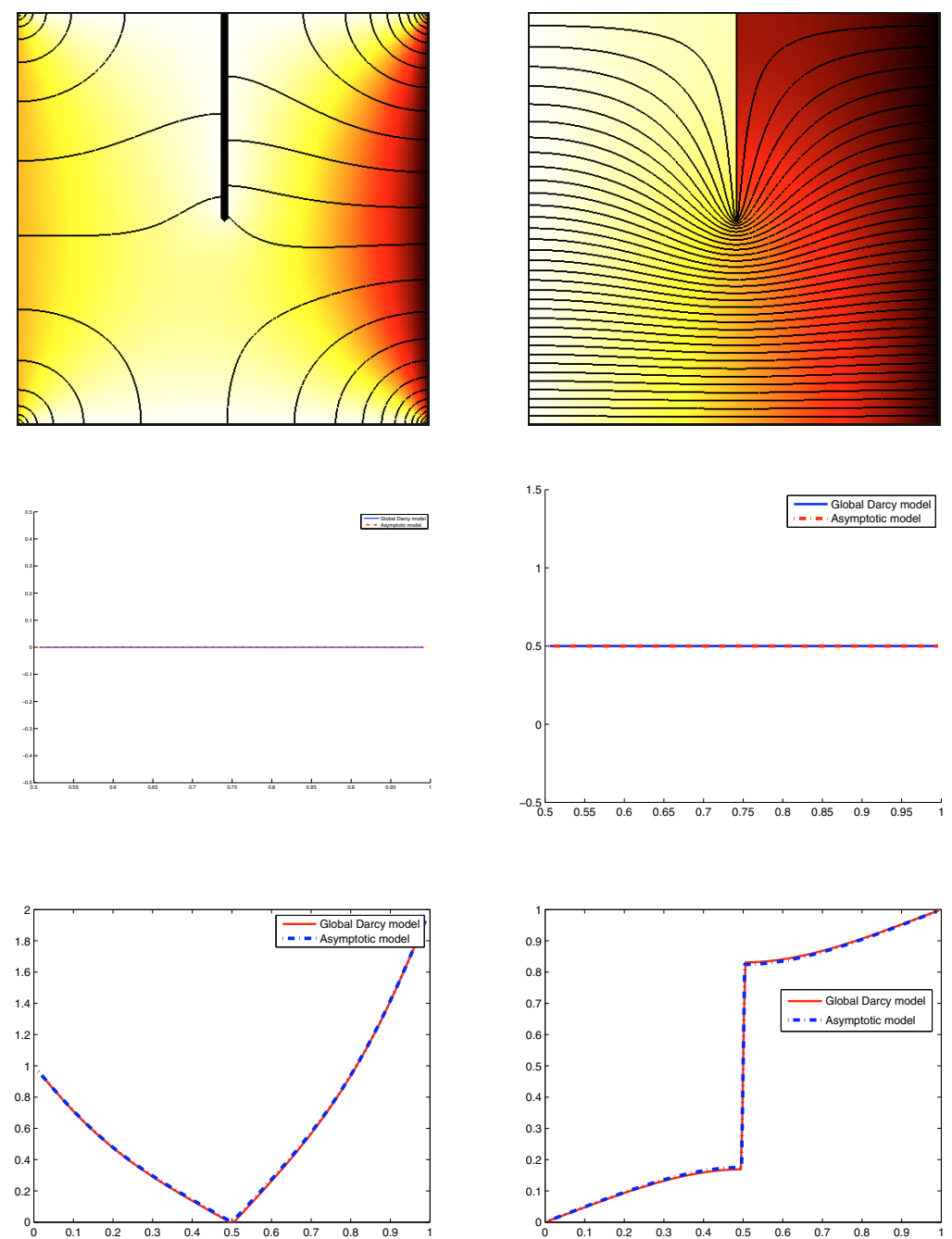

Partially permeable fracture
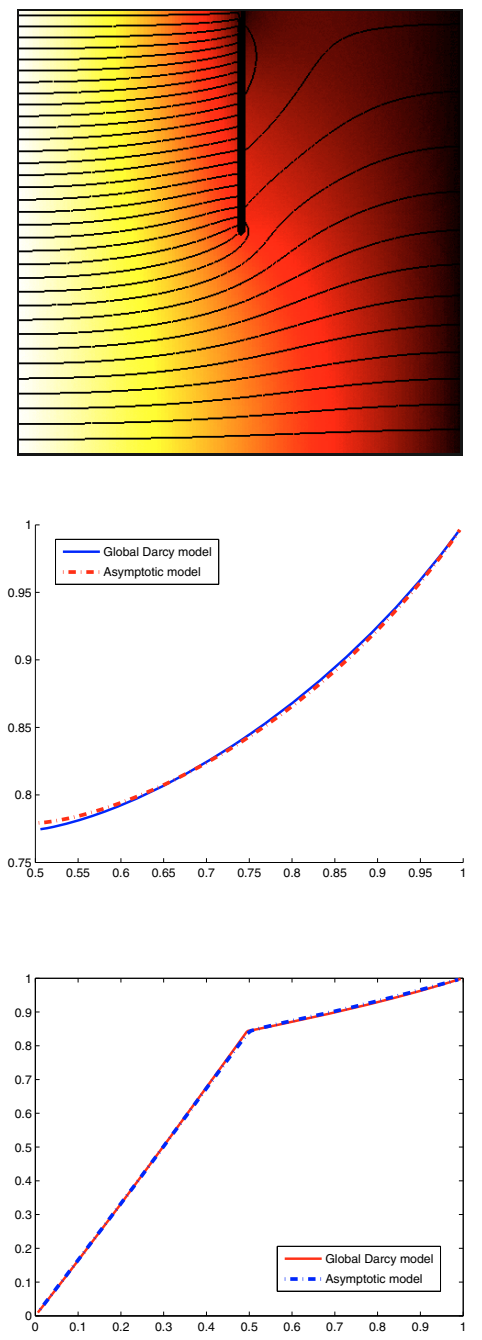

Figure 5. Pressure field and streamlines (top), pressure along the cutline $y=0.75$ (middle), pressure along the fracture (bottom).

In Figures 7 and 8, we show the pressure field obtained with the global Darcy model computed on a mesh containing 65560 rectangular control volumes (that is the reference solution) and the solutions obtained for various values of $\xi$ for the asymptotic model on a mesh with square 16384 control volumes. We first point out that for $\xi<1 / 2$, the asymptotic model may become ill-posed or unstable (in the present situation, negative values of the pressure appear).

In Figures 9 and 10, we go further in the comparisons by showing, on the left part of the figure, the pressure distribution obtained along the fracture in each case. More precisely, we plot $\Pi_{f} p(s)$ for the two models $\xi=1 / 2$ and $\xi=3 / 4$, as well as the quantity $\bar{p}(s)=\left(\gamma^{+} p(s)+\gamma^{-} p(s)\right) / 2$ for $\xi=\frac{3}{4}$. We recall that when $\xi=1 / 2$, we have $\Pi_{f} p=\bar{p}$ that is the reason why there is only one curve in that case. 

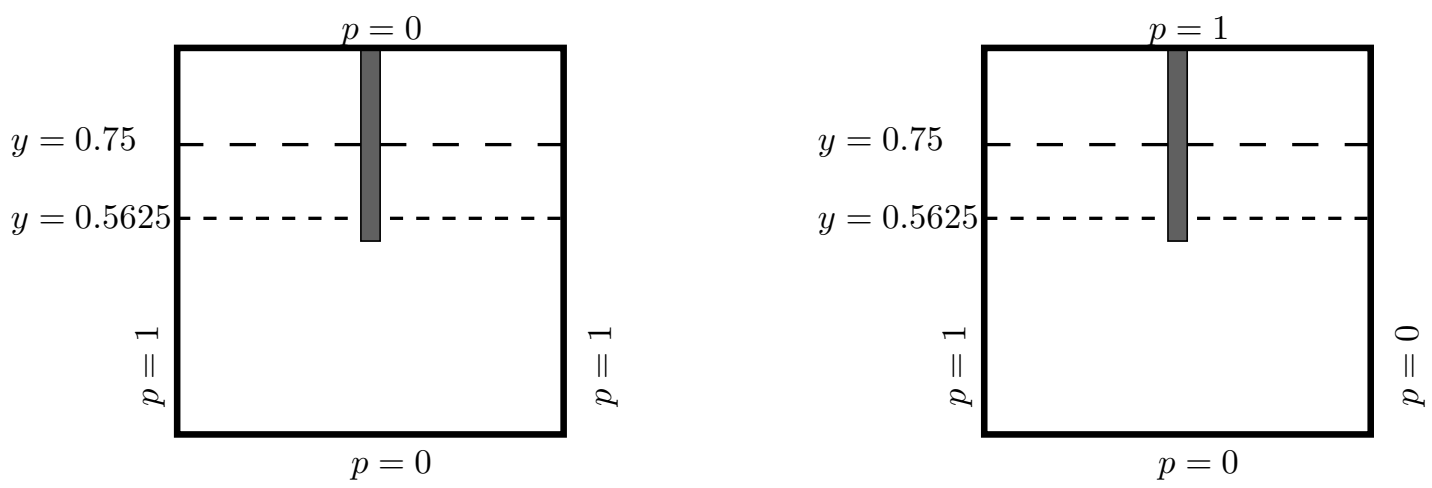

Partially permeable fracture

$$
\mathbf{K}_{f, \boldsymbol{\tau}}=100, \mathbf{K}_{f, \boldsymbol{\nu}}=10^{-4}
$$

FiguRE 6. Boundary condition for a partially permeable fracture. Symmetric flow (left), non symmetric flow (right).

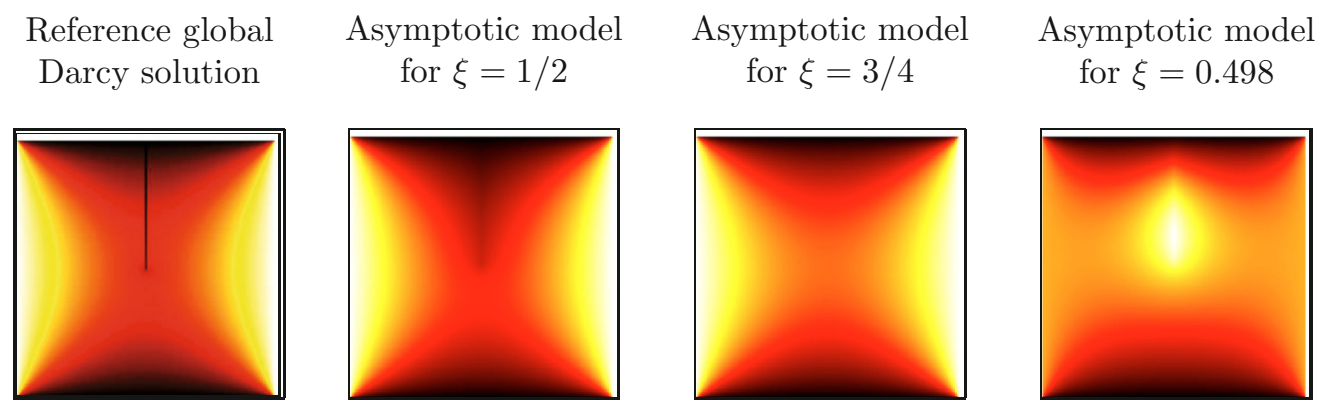

Figure 7. Comparison between the pressure field obtained with the global Darcy model and the asymptotic model for various values of $\xi$ for the symmetric flow.

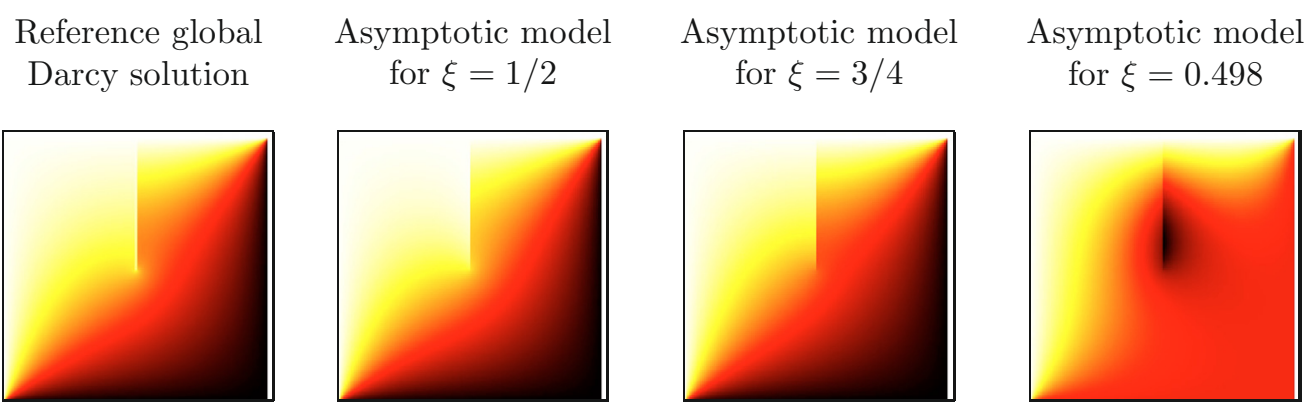

Figure 8. Comparison between the pressure field obtained with the global Darcy model and the asymptotic model for various values of $\xi$ for the non symmetric flow.

The reference mean-pressure $s \mapsto \frac{1}{b_{f}} \int_{-b_{f} / 2}^{b_{f} / 2} p(t, s) \mathrm{d} t$ computed by the resolution of the global Darcy model on the fine grid is also given for reference. These results confirm that the model for $\xi=\frac{1}{2}$ leads to more precise results. The other two parts (center and right) of Figures 9 and 10 show the pressure along two cutlines $y=0.5625$ and $y=0.75$. 

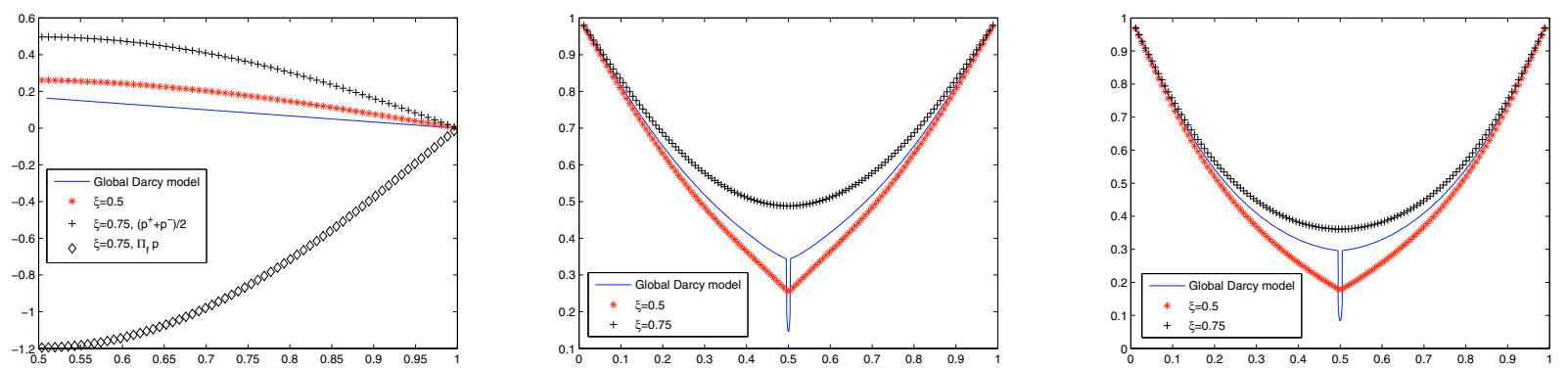

Figure 9. Comparison of pressure along and across the fracture between the global Darcy model and the asymptotic model for $\xi=0.5$ and $\xi=0.75$.

Pressure along the fracture

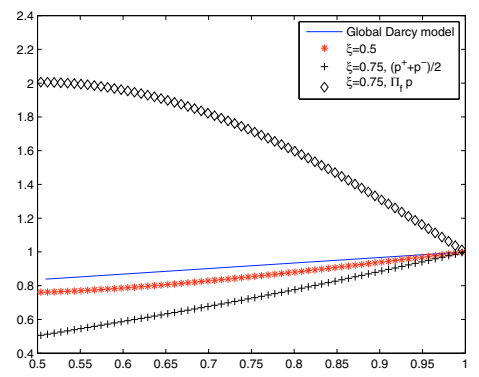

Pressure on the cutline $y=0.5625$

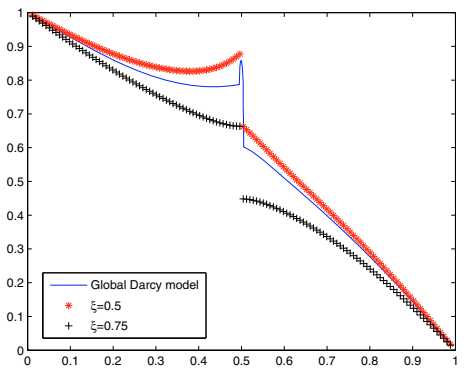

Pressure on the cutline $y=0.75$

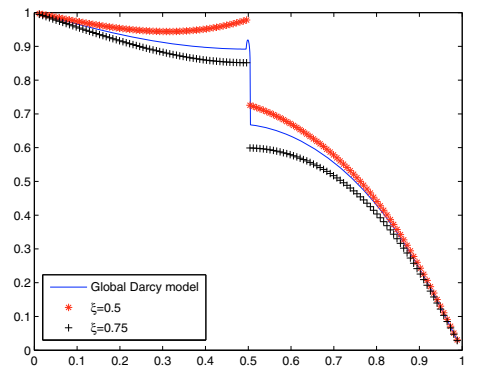

Figure 10. Comparison of pressure along and across the fracture between the global Darcy model and the asymptotic model for $\xi=0.5$ and $\xi=0.75$.

We are not able to draw any definitive conclusion on what should be a good choice for the parameter $\xi$. Nevertheless, it seems that the pressure along the fracture (left-hand side part of Figs. 9 and 10) is more accurately computed by using the simplest model, that is the one with $\xi=1 / 2$. It is also confirmed in these figures that the choice of the no-flux boundary conditions at the extremities of the fracture is an important issue that should be further analysed to improve accuracy of the models.

Finally, we observe that both choices $(\xi=1 / 2$ and $\xi=3 / 4)$ lead to quite accurate pressure computations outside the fracture, at least far enough from the extremities of $\Sigma$ immersed in $\tilde{\Omega}$.

From now on, in the remaining of this paper, we systematically use the value $\xi=1 / 2$ in our computations.

\subsection{Results for a network of half straight fractures}

We consider here a set of four horizontal fractures of aperture $b_{f}=0.01$ defined by $\mathcal{F}_{i}=\Sigma_{i} \times\left[-\frac{b_{f}}{2}, \frac{b_{f}}{2}\right]$, with $\Sigma_{1}=\{(x, y)$ such that $x \geq 0.3, y=0.2\}, \Sigma_{2}=\{(x, y)$ such that $x \leq 0.7, y=0.4\}, \Sigma_{3}=\{(x, y)$ such that $x \geq 0.3, y=0.6\}, \Sigma_{4}=\{(x, y)$ such that $x \leq 0.7, y=0.8\}$. The fractures $\mathcal{F}_{2}$ and $\mathcal{F}_{4}$ are impermeable $\mathbf{K}_{f, \boldsymbol{\tau}}=\mathbf{K}_{f, \boldsymbol{\nu}}=10^{-2}$, whereas $\mathcal{F}_{1}$ and $\mathcal{F}_{3}$ present intermediate properties $\mathbf{K}_{f, \boldsymbol{\tau}} \in\{1,10\}$ and $\mathbf{K}_{f, \boldsymbol{\nu}}=10^{-2}$. For the boundary conditions described in Figure 11, we compare in Figure 12 the solution (solid line) of the global Darcy model using a mesh with 201704 square control volumes, and the solution (dashed line) 


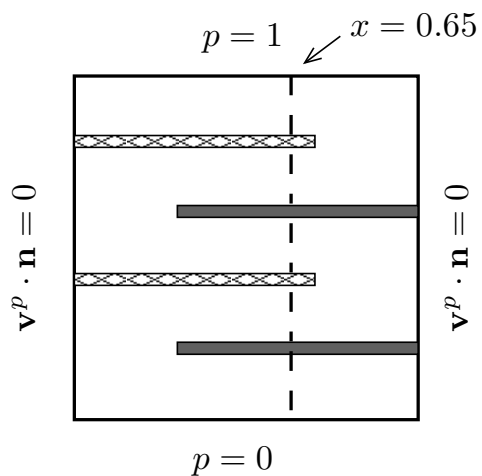

$\mathrm{BC} \mathbf{n}^{o} \mathbf{1}$

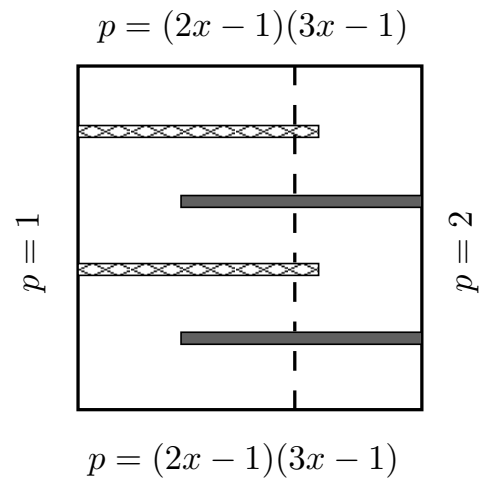

$\mathrm{BC} \mathrm{n}^{\circ} 2$

Figure 11. Configuration and boundary conditions for a network of half straight fractures.

of the asymptotic model, using a mesh with 25600 square control volumes, along the four fractures $\Sigma_{i}$ and along the cutline $\{x=0.65\}$.

We observe a perfect agreement of the two solutions in the first two cases since the curves are almost indistinguishable. In the third case, where $\mathbf{K}_{f, \boldsymbol{\tau}}$ is larger, we can see that we approach the validity limit of the model. It appears here also that the Neuman condition at the boundary of the fracture is not perfectly suitable in this case. A more precise modelling of the asymptotic boundary condition on $\partial \Sigma$ seems to really be necessary in this kind of situations.

\subsection{Fracture with variable aperture}

We now investigate the ability of the asymptotic model to simulate flows in fractures with variable aperture. We consider (see Fig. 13) a vertical conic-shaped fracture at $x=\frac{1}{2}$ where $b_{f}$ varies linearly from 0.001 (bottom) to 0.019 (top). We consider an isotropic case $\mathbf{K}_{f, \boldsymbol{\tau}}=\mathbf{K}_{f, \boldsymbol{\nu}}=100$.

In Figure 14, we compare the results obtained by the global Darcy model (left part of the figure) and by the asymptotic model (center part of the figure). We can see a quite good agreement. Notice that the mesh used to compute the global Darcy solution has to be very fine near the bottom of the fracture since its aperture is equal to $10^{-3}$ in this area. More precisely, the asymptotic results presented here have been obtained for a rectangular mesh with 16364 control volumes, whereas the global Darcy computation has been performed on a triangular mesh with 96764 control volumes, suitably refined in the neighbourhood of the fracture.

We also give for comparison, the result obtained with the asymptotic model if we replace the variable (linear) aperture $b_{f}(s)$ with a constant mean-value $b_{f}(s) \equiv 10^{-2}$, which amounts to approximating the conic-shaped fracture with an "equivalent" constant aperture fracture. We clearly see that the result is less satisfactory which demonstrates the need to suitably take into account the geometry of the fracture in the asymptotic model.

\subsection{Comparison with an analytic solution}

We finally test the model in the case of a permeable lens-shaped fracture, using the analytic behaviour of the flow obtained in [23] in the neighbourhood of the extremities of the lens embedded in an infinite porous medium. This lens, centered at $(0.5,0.5)$ and whose length is 0.2 , is limited by the arcs of two circles of the same radius meeting at a right angle. We refer to Figure 15 which also presents the boundary conditions used for the numerical simulations. We consider the isotropic case where $\mathbf{K}_{f, \boldsymbol{\tau}}=\mathbf{K}_{f, \boldsymbol{\nu}}=K_{f}=12$. For a flow parallel 
Boundary conditions 1

$\mathbf{K}_{f, \boldsymbol{\tau}}=10^{2}$ on $\mathcal{F}_{1}$ and $\mathcal{F}_{3}$
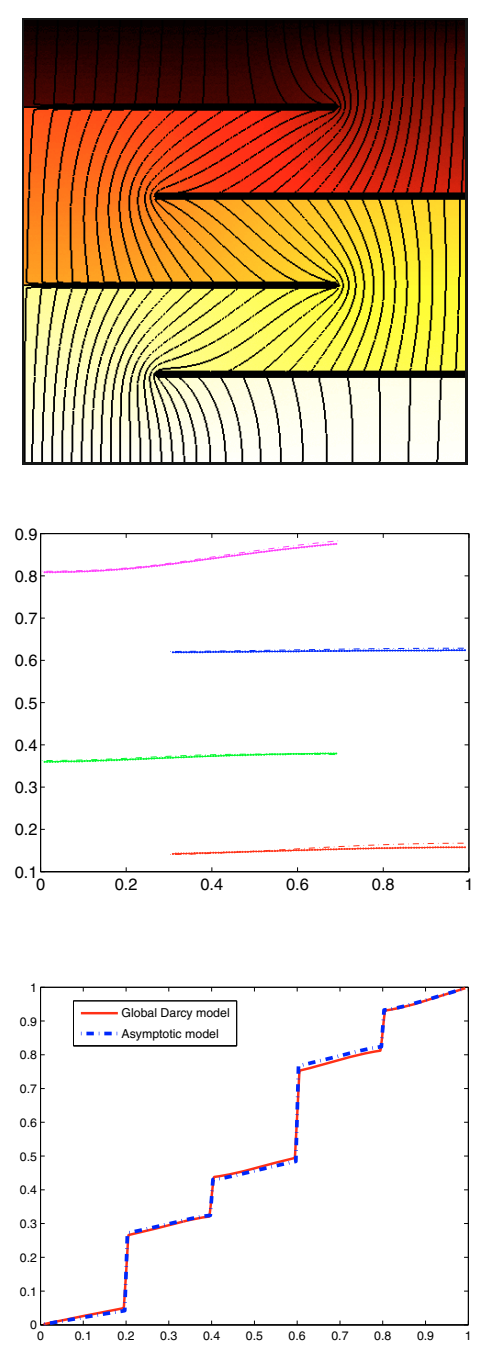

Boundary conditions 2

$\mathbf{K}_{f, \boldsymbol{\tau}}=1$ on $\mathcal{F}_{1}$ and $\mathcal{F}_{3}$
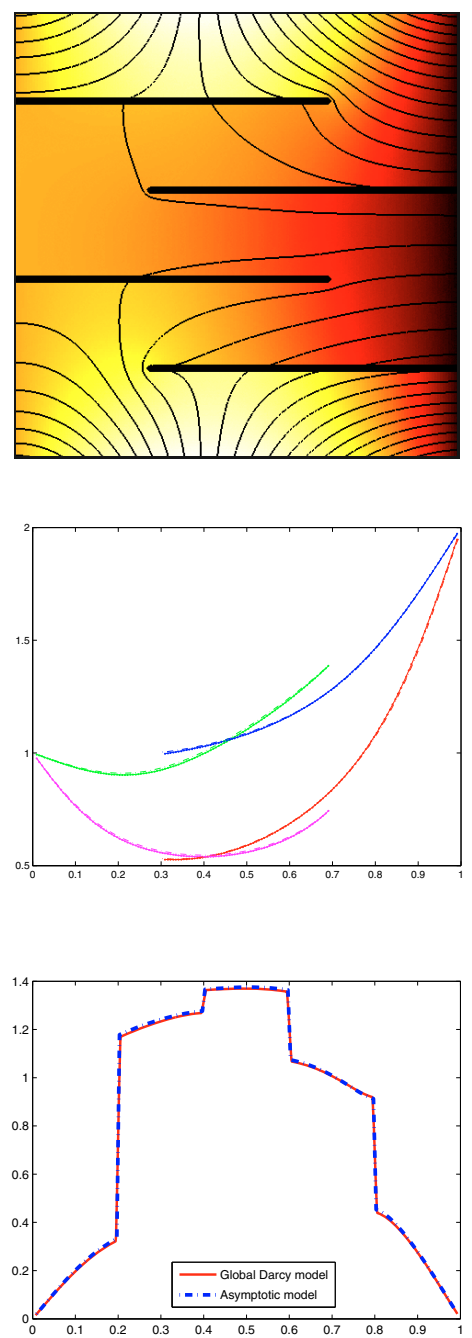

Boundary conditions 2

$\mathbf{K}_{f, \boldsymbol{\tau}}=10$ on $\mathcal{F}_{1}$ and $\mathcal{F}_{3}$
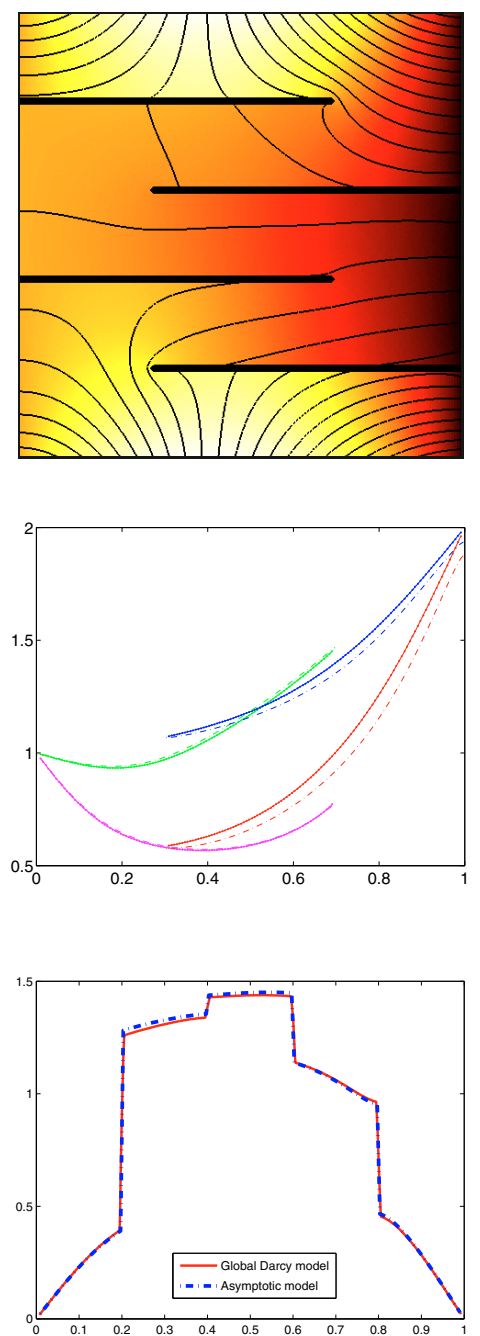

Figure 12. Pressure field and streamlines (top), pressure along the four fractures (middle), pressure along the cross section $\Sigma_{\perp}$ (bottom).

to the main symmetry axis of the lens, the analytic solution derived in [23] in the neighbourhood of the extremity $(0.5,0.6)$, is equivalent up to a constant to

$$
p^{\text {anal }}(y) \sim \begin{cases}\frac{4}{\left(K_{f}+1\right) \Delta}\left(\frac{0.6-y}{y-0.4}\right)^{\gamma_{1}}, & y<0.6, \\ \frac{2}{\Delta}\left(\cos \left(\pi \gamma_{1}\right)-\frac{K_{f}-1}{K_{f}+1} \cos \left(\frac{\pi \gamma_{1}}{2}\right)\right)\left(\frac{y-0.6}{y-0.4}\right)^{\gamma_{1}}, & y \geq 0.6,\end{cases}
$$

with $\Delta=\cos \left(\pi \gamma_{1}\right)-\frac{1}{2} \frac{K_{f}-1}{K_{f}+1} \cos \left(\frac{\pi \gamma_{1}}{2}\right), \gamma_{1}=\frac{2}{\pi} \operatorname{acos}\left(\frac{1}{2} \frac{K_{f}-1}{K_{f}+1}\right)$. 


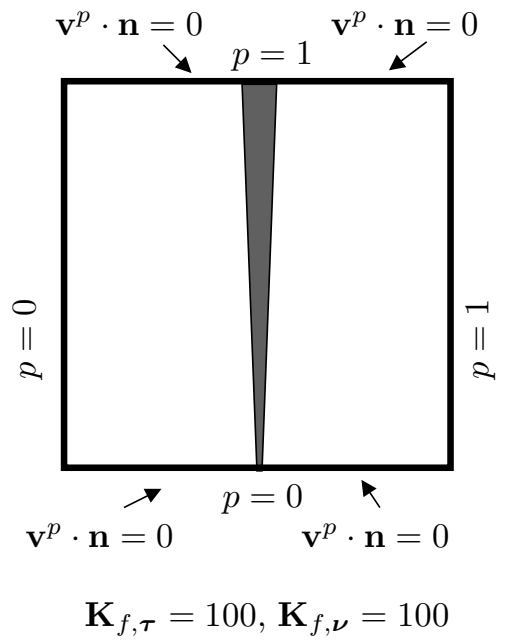

Figure 13. Configuration and boundary conditions for the conic-shaped fracture.

Global Darcy model

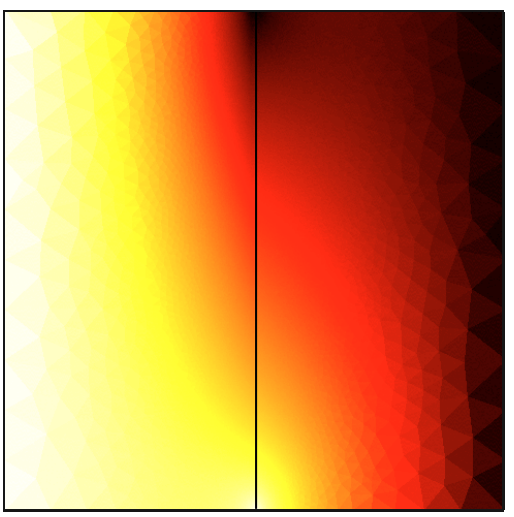

Asymptotic model taking into account the variable aperture

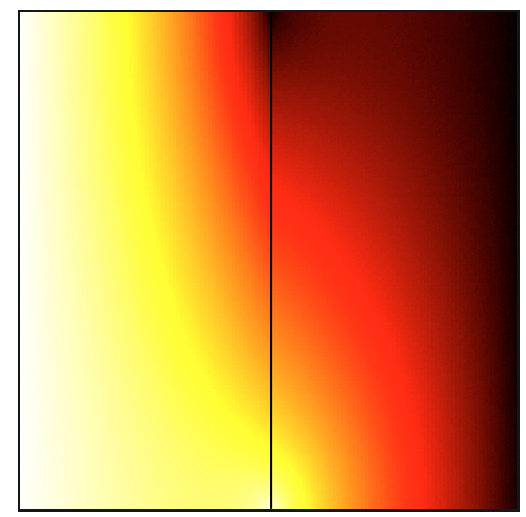

Asymptotic model for a constant aperture fracture

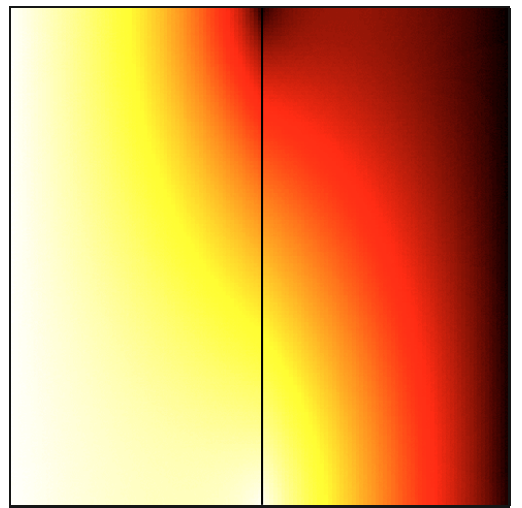

FiguRE 14. Influence of the shape of the fracture.

The right part of Figure 15 shows the good agreement near the extremity between this analytic solution and the global Darcy solution, computed on a triangular mesh with 19848 cells. Note that we chose for the additive constant the pressure value given by the global Darcy solution at the angular point $(0.5,0.6)$. The solution given by the asymptotic model on a mesh composed of 13448 triangles is globally close to the global Darcy solution, except in the very neighbourhood of the extremity of the lens, where the aperture vanishes. Since the aperture (s) vanishes at the two extremities of the fracture, this test case does not exactly enter the analysis proposed in this paper. Nevertheless, the model seems to give satisfactory results even in this situation.

\subsection{Extension to more general geometries}

We conclude this section by showing some results in more general geometric situations. Figure 16 shows the pressure fields obtained in the case of a circular fracture and in the case of a polygonal fracture. We observe a good qualitative agreement between the solution computed by the asymptotic model under study and 

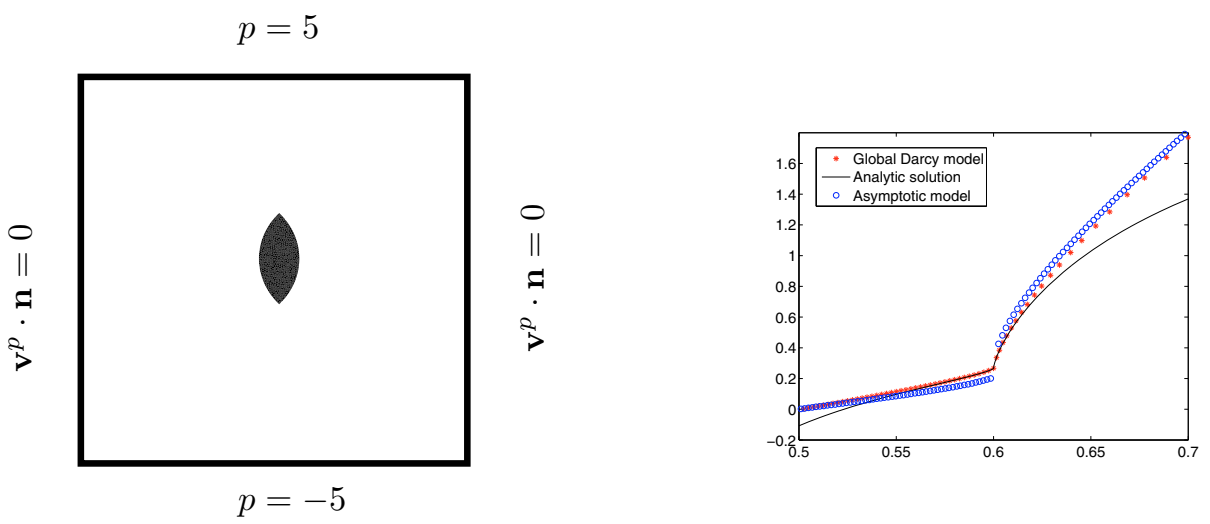

Figure 15. The lens-shaped fracture: Configuration and boundary conditions (left), pressure field along the symmetry axis $x=0.5$ of the fracture near one of its extremity (right).

the one obtained by solving the reference global Darcy model. Note that, in the case of the circular fracture, the influence of the curvature of $\Sigma$ has to be taken into account in the derivation of the model. Here, since this curvature is constant, it only amounts to add a constant multiplicative factor in front of the tangential divergence operator in equation $\left(\mathcal{P}_{\mathcal{A}}\right.$-e).

\section{Conclusion And Perspectives}

In this study, some asymptotic models of flow in 2D fractured porous media are formally derived in the case where the fracture domains, whose aperture is supposed to be small, are reduced to polygonal interfaces immersed inside the porous matrix. It is then needed to solve an adequate 1D Darcy-type equation along these immersed interfaces, coupled with an usual 2D Darcy equation in the porous matrix.

A cell-centered finite volume scheme is investigated to solve this coupled problem. The existence of solutions to the asymptotic models is proved by first showing existence of the solution of the finite volume approximation schemes and then showing convergence of these solutions to the solution of the continuous problem when the mesh size tends to 0 . Uniqueness of such a solution is ensured by energy estimates. The convergence proof of the scheme is quite intricate due to the fact that, because of the immersed interfaces, the domain under study has cuts, and then is not located on a unique side of its boundary.

Using the proposed numerical scheme, the behavior and the validity of the asymptotic models are then investigated for a large variety of situations. For these examples, we obtain a good agreement with the solutions computed analytically or obtained by using appropriate numerical schemes for the global Darcy problem on locally refined meshes. In our experiments, the number of required degrees of freedom saved with the use of the asymptotic models proves to be all the more important when the permeability jumps are large.

In a further work we should investigate the rigorous justification of the asymptotic model, by showing that the solutions of the global Darcy problem and of the asymptotic model are close enough $\left(\operatorname{say} O\left(b_{f}^{2} /|\Sigma|^{2}\right)\right)$ in a relevant functional space. The models and the numerical methods studied here could be generalised to $3 \mathrm{D}$ fractured porous media, at least for simple fracture geometries like planar polygonal ones.

It would be interesting to investigate the coupling of such models with a time dependent advection-diffusion equation for the solutal transport in order to study solutal dispersion phenomena in fractured porous media. Another perspective could be to consider fractures in which the flow is governed by the Stokes equation instead of the Darcy one. 
Global Darcy model

Circular fracture

$\mathbf{K}_{f, \boldsymbol{\tau}}=\mathbf{K}_{f, \boldsymbol{\nu}}=10^{-4}$

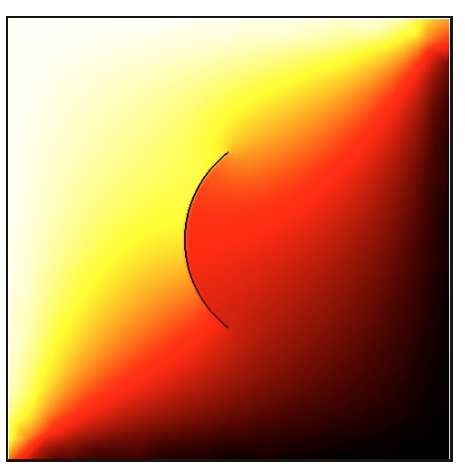

Asymptotic model

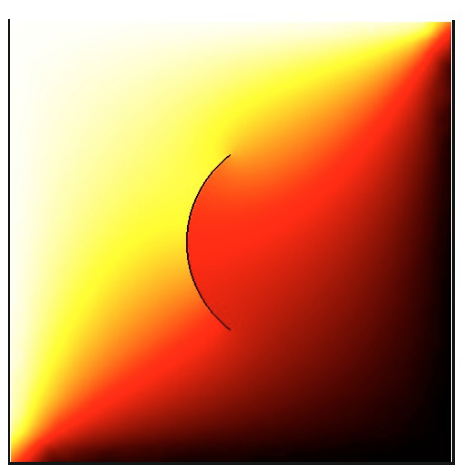

Polygonal fracture $\mathbf{K}_{f, \boldsymbol{\tau}}=10^{4}, \mathbf{K}_{f, \boldsymbol{\nu}}=10^{-4}$
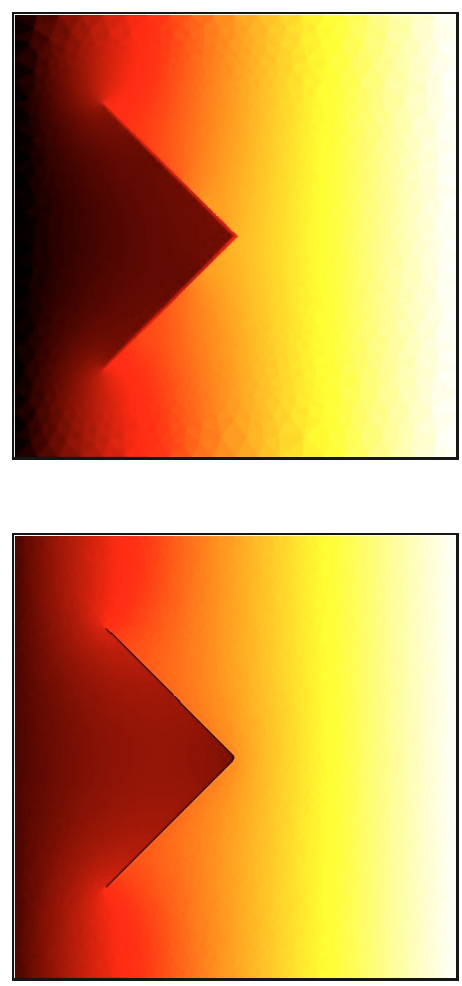

Figure 16. Pressure fields for a circular fracture and a polygonal fracture. Comparison between the reference solution (global Darcy model) and the solution of the asymptotic model.

\section{A. Appendix: Proof of a technical Result}

Proof of the density result in Proposition 3.1. Let us give a proof in the simple case where $\tilde{\Omega}=\mathbb{R}^{2}, \Sigma=[0,1] \times$ $\{0\}$, and $\Omega=\tilde{\Omega} \backslash \Sigma$. We choose $\Omega^{+}=\mathbb{R} \times \mathbb{R}^{+}$and $\Omega^{-}=\mathbb{R} \times \mathbb{R}^{-}$. Let $v \in H^{1}(\Omega)$ such that $\gamma_{\Sigma} v=0$, that is to say $\gamma^{+} v=\gamma^{-} v=0$ on $\Sigma$.

First of all, since we have the equality $\gamma^{+} v=\gamma^{-} v$ on the whole real line $\mathbb{R} \times\{0\}$ we see that $v \in H^{1}\left(\mathbb{R}^{2}\right)$.

Let $\varepsilon>0$. Recall that single point sets have a zero $H^{1}$-capacity in $\mathbb{R}^{2}$. In particular, there exists $\eta>0$ and $w \in H^{1}\left(\mathbb{R}^{2}\right)$ such that $\|v-w\|_{H^{1}\left(\mathbb{R}^{2}\right)} \leq \varepsilon$ and $w=0$ identically on the two balls $B((0,0), \eta), B((1,0), \eta)$ centered at the two extremities of $\Sigma$. Furthermore, we can choose $w$ in such a way that $\gamma^{+} w=\gamma^{-} w=0$ on $\Sigma$. Hence, we have $\gamma^{+} w=\gamma^{-} w=0$ on $[-\eta, 1+\eta] \times\{0\}$. Let now $x \in \mathbb{R} \mapsto \phi_{1}(x)$ be a smooth function with support in $]-\eta, 1+\eta\left[\right.$ and such that $\phi_{1}(x)=1$ for $x \in[-\eta / 2,1+\eta / 2]$, and let $y \in \mathbb{R} \mapsto \phi_{2}(y)$ be another smooth function with support in ]-1, 1 [ and such that $\phi_{2}(y)=1$ for $y \in[-1 / 2,1 / 2]$.

For any $\delta>0$ we define $v_{\delta}(x, y)=w(x, y) \times\left(1-\phi_{1}(x) \phi_{2}(y / \delta)\right)$ and we claim that $v_{\delta}$ converges to $w$ in $H^{1}\left(\mathbb{R}^{2}\right)$ when $\delta$ goes to 0 . The convergence of $v_{\delta}$ and $\partial_{x} v_{\delta}$ in $L^{2}\left(\mathbb{R}^{2}\right)$ towards $w$ and $\partial_{x} w$ respectively is straightforward by using the Lebesgue theorem. Let us now show the convergence of $\partial_{y} v_{\delta}$ when $\delta$ goes to 0 . To this end, the only non trivial term to estimate is

$$
T_{\delta}=\int_{-\eta}^{1+\eta} \int_{-\delta}^{\delta}\left|w(x, y) \phi_{1}(x)\right|^{2} \frac{1}{\delta^{2}}\left|\phi_{2}^{\prime}(y / \delta)\right|^{2} \mathrm{~d} x \mathrm{~d} y
$$


Since $\phi_{1}$ and $\phi_{2}^{\prime}$ are bounded we have

$$
T_{\delta} \leq C \int_{-\eta}^{1+\eta} \int_{-\delta}^{\delta} \frac{1}{\delta^{2}}|w(x, y)|^{2} \mathrm{~d} x \mathrm{~d} y \leq C \int_{-\eta}^{1+\eta} \int_{-\delta}^{\delta} \frac{1}{y^{2}}|w(x, y)|^{2} \mathrm{~d} x \mathrm{~d} y .
$$

Since $\gamma^{+} w=\gamma^{-} w=0$ for any $x \in[-\eta, 1+\eta]$, we can use the Hardy inequality and obtain

$$
T_{\delta} \leq C \int_{-\eta}^{1+\eta} \int_{-\delta}^{\delta}\left|\partial_{y} w(x, y)\right|^{2} \mathrm{~d} x \mathrm{~d} y
$$

and we finally find that $T_{\delta} \rightarrow 0$ when $\delta \rightarrow 0$ by the Lebesgue theorem.

As a conclusion, we can find $\delta$ small enough such that $\left\|v_{\delta}-w\right\|_{H^{1}\left(\mathbb{R}^{2}\right)} \leq \varepsilon$. Gathering the previous results we have

$$
\left\|v-v_{\delta}\right\|_{H^{1}\left(\mathbb{R}^{2}\right)} \leq\|v-w\|_{H^{1}\left(\mathbb{R}^{2}\right)}+\left\|w-v_{\delta}\right\|_{H^{1}\left(\mathbb{R}^{2}\right)} \leq 2 \varepsilon .
$$

By construction, we see that $v_{\delta}$ is identically zero on $[-\eta / 2,1+\eta / 2] \times[-\delta / 2, \delta / 2]$, hence by convolution with a suitable regularization kernel with compact support we may find a smooth function $\phi_{\varepsilon}$ which is identically zero on $[-\eta / 4,1+\eta / 4] \times[-\delta / 4, \delta / 4]$ and such that $\left\|v_{\delta}-\phi_{\varepsilon}\right\|_{H^{1}\left(\mathbb{R}^{2}\right)} \leq \varepsilon$.

We finally found a function $\phi_{\varepsilon} \in \mathcal{C}_{c}^{\infty}(\Omega)$ (since $\Sigma$ does not intersect the support of $\phi_{\varepsilon}$ ) and such that $\left\|v-\phi_{\varepsilon}\right\|_{H^{1}(\Omega)} \leq 3 \varepsilon$, and then the claim is proved.

Acknowledgements. The authors want to thank P. Adler for many stimulating discussions and the anonymous referees for their very careful reading of this paper. This study has been carried out in the framework of the research project "Dispersion in fractured porous media" which received a financial support from the GdR CNRS MoMaS in the period 2004-2006.

\section{REFERENCES}

[1] P.M. Adler and J.-F. Thovert, Fractures and Fracture Networks. Kluwer Acad., Amsterdam (1999).

[2] B. Andreianov, F. Boyer and F. Hubert, Discrete duality finite volume schemes for Leray-Lions type elliptic problems on general 2D-meshes. Numer. Methods Partial Differential Equations 23 (2007) 145-195.

[3] P. Angot, Finite volume methods for non smooth solution of diffusion models; application to imperfect contact problems, in Recent Advances in Numerical Methods and Applications, O.P. Iliev, M.S. Kaschiev, S.D. Margenov, B.H. Sendov and P.S. Vassilevski Eds., Proc. 4th Int. Conf. NMA'98, Sofia (Bulgarie), World Sci. Pub. (1999) 621-629.

[4] P. Angot, A model of fracture for elliptic problems with flux and solution jumps. C. R. Acad. Sci. Paris Ser. I Math. 337 (2003) 425-430.

[5] P. Angot, T. Gallouët and R. Herbin, Convergence of finite volume methods on general meshes for non smooth solution of elliptic problems with cracks, in Finite Volumes for Complex Applications II, R. Vilsmeier, F. Benkhaldoun and D. Hänel Eds., Hermès (1999) 215-222.

[6] J. Bear, C.-F. Tsang and G. de Marsily, Flow and Contaminant Transport in Fractured Rock. Academic Press, San Diego (1993).

[7] B. Berkowitz, Characterizing flow and transport in fractured geological media: A review. Adv. Water Resour. 25 (2002) 861-884.

[8] C. Bernardi, M. Dauge and Y. Maday, Compatibilité de traces aux arêtes et coins d'un polyhèdre. C. R. Acad. Sci. Paris Sér. I Math. 331 (2000) 679-684.

[9] C. Bernardi, M. Dauge and Y. Maday, Polynomials in the Sobolev world. (2007) http://hal.archives-ouvertes.fr/ hal-00153795.

[10] I.I. Bogdanov, V.V. Mourzenko, J.-F. Thovert and P.M. Adler, Effective permeability of fractured porous media in steady-state flow. Water Resour. Res. 107 (2002).

[11] F. Boyer and F. Hubert, Finite volume method for 2D linear and nonlinear elliptic problems with discontinuities. SIAM J. Numer. Anal. 46 (2008) 3032-3070.

[12] Y. Caillabet, P. Fabrie, P. Landereau, B. Noetinger and M. Quintard, Implementation of a finite-volume method for the determination of effective parameters in fissured porous media. Numer. Methods Partial Differential Equations 6 (2000) 237-263. 
[13] Y. Caillabet, P. Fabrie, D. Lasseux and M. Quintard, Computation of large-scale parameters for dispersion in fissured porous medium using finite-volume method. Comput. Geosci. 5 (2001) 121-150.

[14] K. Domelevo and P. Omnes, A finite volume method for the Laplace equation on almost arbitrary two-dimensional grids. ESAIM: M2AN 39 (2005) 1203-1249.

[15] R. Eymard and T. Gallouët, H-convergence and numerical schemes for elliptic equations. SIAM J. Numer. Anal. 41 (2003) $539-562$.

[16] R. Eymard, T. Gallouët and R. Herbin, Finite Volume Methods, in Handbook of Numerical Analysis VII, P.G. Ciarlet and J.L. Lions Eds., North-Holland (2000) 713-1020.

[17] I. Faille, E. Flauraud, F. Nataf, S. Pégaz-Fiornet, F. Schneider and F. Willien, A new fault model in geological basin modelling. Application of finite volume scheme and domain decomposition methods, in Finite Volumes for Complex Applications III, R. Herbin and D. Kröner Eds., Hermes Penton Sci. (HPS) (2002) 543-550.

[18] B. Faybishenko, P.A. Witherspoon and S.M. Benson, Dynamics of Fluids in Fractured Rock, Geophysical Monograph Series 122. American Geophysical Union, Washington D.C. (2000).

[19] P. Grisvard, Elliptic problems in nonsmooth domains, Monographs and Studies in Mathematics 24. Pitman, Advanced Publishing Program, Boston (1985).

[20] F. Hermeline, Approximation of diffusion operators with discontinuous tensor coefficients on distorted meshes. Comput. Methods Appl. Mech. Engrg. 192 (2003) 1939-1959.

[21] J. Jaffré, V. Martin and J.E. Roberts, Generalized cell-centered finite volume methods for flow in porous media with faults, in Finite Volumes for Complex Applications III, R. Herbin and D. Kröner Eds., Hermes Penton Sci. (HPS) (2002) 357-364.

[22] V. Martin, J. Jaffré and J.E. Roberts, Modeling fractures and barriers as interfaces for flow in porous media. SIAM J. Sci. Comput. 26 (2005) 1667-1691.

[23] V. Mityushev and P.M. Adler, Darcy flow arround a two dimensional lense. Journal Phys. A: Math. Gen. 39 (2006) 3545-3560.

[24] V. Reichenberger, H. Jakobs, P. Bastian and R. Helmig, A mixed-dimensional finite volume method for two-phase flow in fractured porous media. Adv. Water Resour. 29 (2006) 1020-1036. 